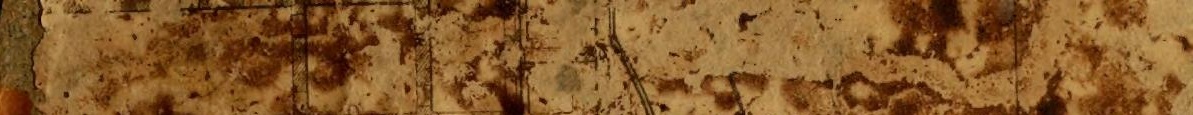

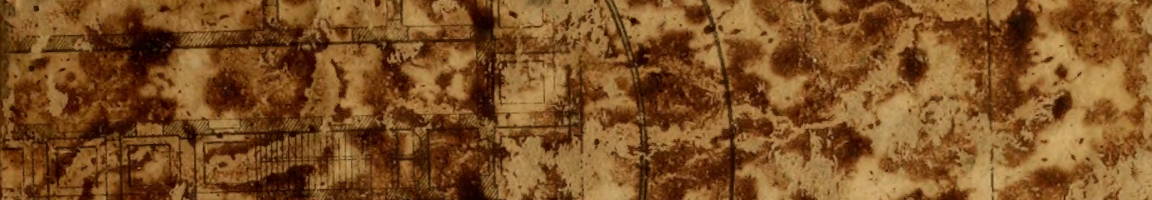

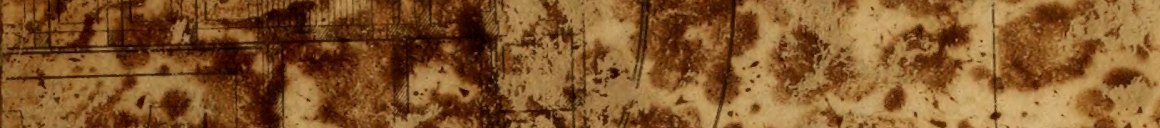

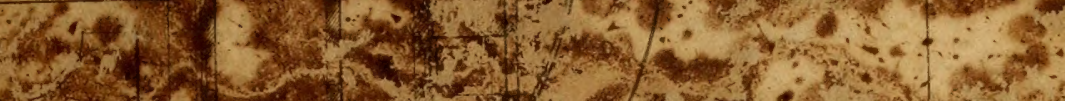

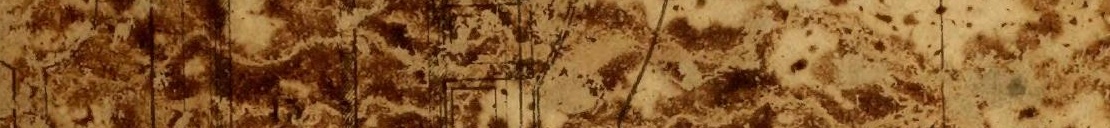

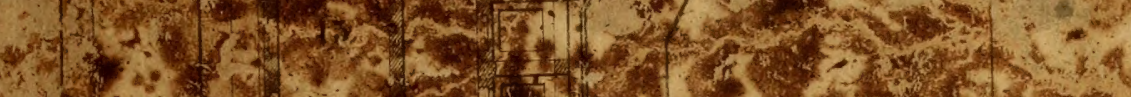

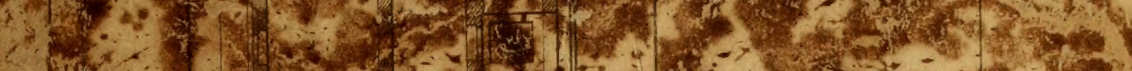

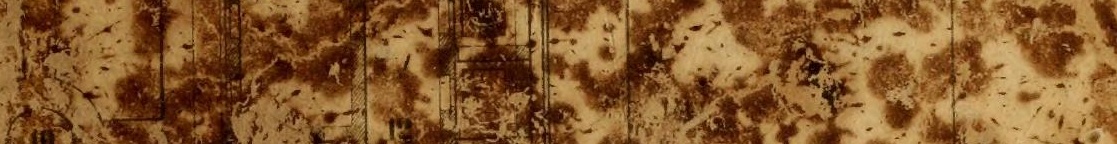
P.t.

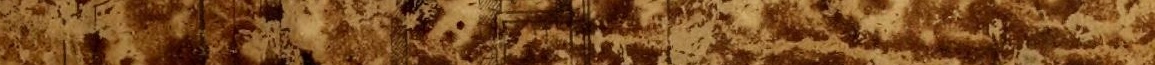
(5)

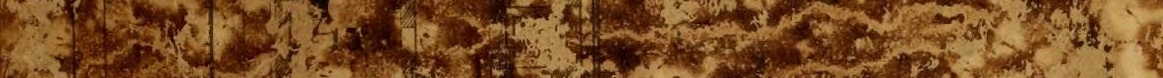

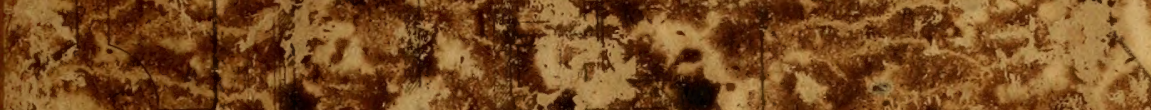

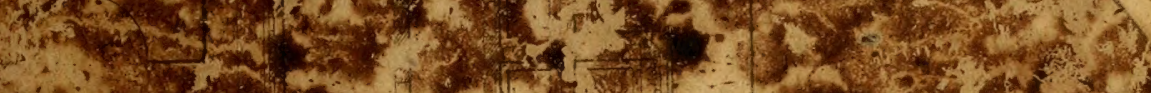

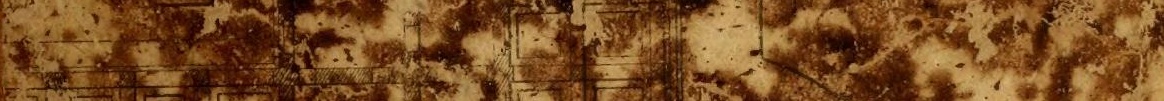

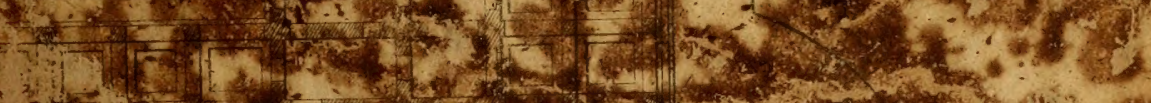

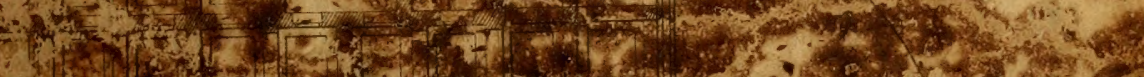

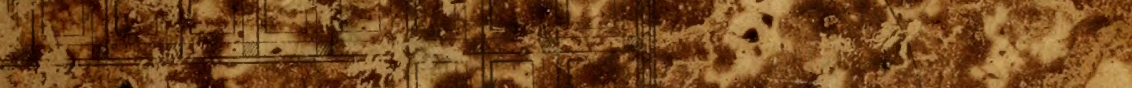
5.0. 3
4

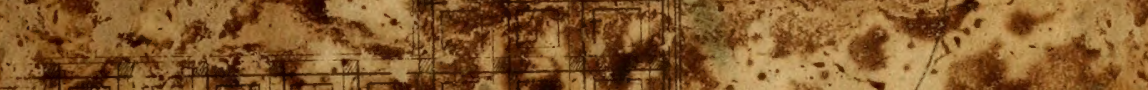

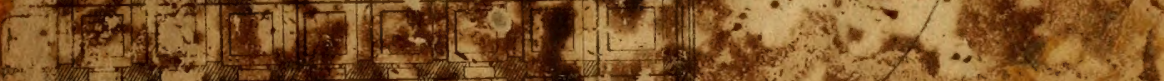

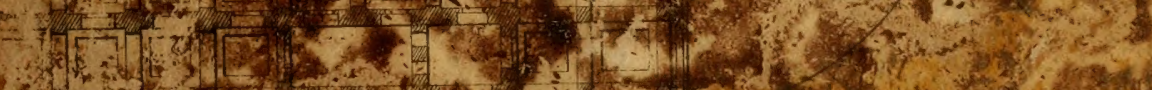

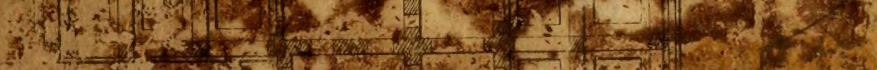

(1) 





\section{ELENGO}

BIRDS DEGI U C CELII

che trovansi nell'Isola di Sardegna

OD

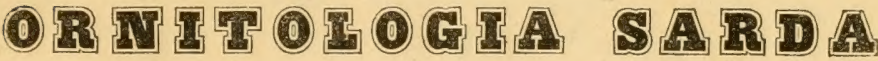

D I

\section{GAPTAYO BABA}

INCARICATO DELLA DIREZIONE DEL R. MUSEO

DI STORIA NATURALE E D'ANTICHITA'

DELL'UNIVERSITA' DI CAGLIARI

Slembro corrispondente di varie Societa Scientifiche

40179 of edicate

1.

S. A. R. VITTORHO EHANUELE

Guca di Sacoir

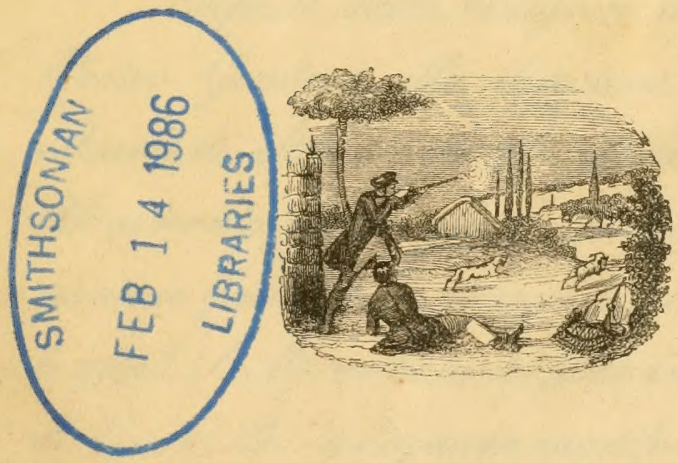

TORINO,

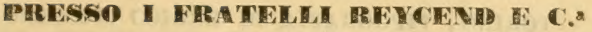

Librai di S. S. R. M.

1842 


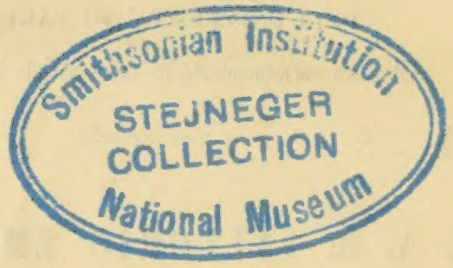

TORINO, TIPOGRAFIA DI ENRICO MUSSANO

Contrada di S. Francesco d'Assisi. 


\section{eldezra Reale,}

Prepasta dalla benignità dell 'Stugusto Vastra Genitare alla direzione del Poegia Mbusea di Mroria naturale e di antichita della Abnivensità di Cagliari, se gran consolazione ia gustai nelle graziasifime parale, con le quali S. I6. nella visita fatta in compragnia di V. 6. R., sidegnava manifestarmi il sua Caurana yradimenta per la mia sollecitudine nel riardinamenta dilutti gli aggetti, de quali: 
e'ricca quella Stabilimenta, mi sentrïure dalle medesime maggiarmente animata a cantinuare nella esecurione dell ufficia cammefromi, ed a facilitare agli amatori delle case naturali la sludia delle medesime.

Intenta a questa fine ha ricaminciata la descrizione delle varie parti di questa Mabilimenta, ed ecca ara fresenta a $\mathscr{U}$. A. R. la Moda che feci secanda la clafoificazione qia veduta da V.S. R., di Hultigli Abccellifinare canasciuti, che nel Roegna di Sardegna a wi dimarana stabilmente, a vi si saglian mastraxe came peregrini in cerle stagiane.

Ta vegga che quest ámagyia nan è quanta merital'A. Q. R. e quantavarebbe il miacuare piena di micanoscenza pe'beneficie, de'quali fu meca per sola sua benignitia liberalifsima la Mbaestic dell' Murususta Qustra Genitare. nan pertanta canfidache l'. 6. U. R. si campiacenai accaglierla fararevalnente. Ciäsara per me un patentifima impulso, o un forte 
incaraggiamenta a proseguire can axdare sempre criescente ne'miei studii per cassi = spandere quanta la permettana le mie farese alla canfidenza che it Tostro bugusta Ge= nitare ripase in me, quanda mi destinava al pasta in ciei sona.

Oi Tostra Oltezza Reale,

L'Umiimo, Devotmo ed Obhed ${ }^{\text {mo }}$ Servitore, 



\section{PREFAZIONE.}

S yia da hunga pezan che in meditavo sul comodo che tornerebbe agli Ornitologi du una raccolta di unte le specie di $L$ ccelli, che popolano inostri campi, le valli, i colli, e lemontagne; che svolazsano nei nostri giardini, che passeggiano sui nostri telli; che motano nelle acque dei nostri mari, fumi, e stagni; " che abitano melle paludi, e giuncaje, siano essi di stabile domicilio, siano di soggiorno temporario a generarvi e crescervi la prole, siano finalmente di passaggio accidentale, che non vi facciano che una breve slazione: e già proponevomi di compilarne un Elenco, in cui dessi comparissero schierati solto li rispettivi ordini, e generi, giusta la classificasione fattane da quello fra i classici, cui piaceami dare la preferenza. 
Ma se da un lato confortacami l'animo l'idea di siffatto comodo, e forse anche l'utile della stessa scienza, dall' altro diverse difficolta mi tenevano esitante ad effettuare il mio dirisamento, finchè non le aressi superale; anzi scoraggiacami quasi affatto il riflesso, che non arendomi fun allora le circostanse di famiglia, e quelle del mio ufficio permesso di fare una perlustrazione generale del Regno, potero per aventura lasciarmi indurre in qualche errore, stabiliendo l'esistensa di alcune specic she non si fossero mai piu viste in Sardegna, oppure trascurando tali altre che fossero gia conosciute.

Aspettavo percio, che altri in posizione pui fortunata della mia prestasse questo servizio alla scienza; oppure rhe cambiale in meglio le mie condizioni potessi far un viaggio in tutte le provincie del Regno, e osservare, studiare, e passare in rassegna rigorosa tutte le specie degli alati, che sono nella Sardegna.

Intanto moltiplicaro sempre piit le mie brevi gite, e parziali perlustrazioni, ripetero le osservasioni, ed aumentaro le mie relasioni con persone stabilite in provincia che mi furono rortesi di esate relazioni, e frequenti spedizioni, pri lo quali di molto si accrebbe il deposito delle mie memorie ormitoloygiche.

Parra forse, che dopo questo io mi sarei potuto credere gii percemuto al punto di poter stabilire son certu cognizione di causa l'esistenza di serle specie, delle quali per lo passalo neppur sospeltaro, e l'assensa dialtre, delle quali stimano arer redute certissime le traccie.

Non pertanto esitavo ancorta act achurare alle stampe un laroro, che credea ancora imperfetto, findie le amirheroli solle- 
citudini del prestantissimo Marchese C'arlo Duraz:o assiduo cultore di questa parte della storia naturale, e l'autorevole consiglio del Chiarissimo Cavaliere Alberto Della Marmora, cui debbo co' mici comnazionali un rispettoso tributo di riconoscenza per avere colle sue lunghe, e penose fatiche messo in rista questo paese, quale sul rapporto di storia naturale, e di antichita conosrevasi appena, e T'esempio del celebre professore C. 1. Temminck che non sdegnò rettificare posteriormente diverse imperfezioni alle quali ando soggento il suo Manuel Omilhologique. mi diedero un potente impulso ad ordinare le raccolte memorie. ed applicarmi di proposilo, e con coraggio al divisalo mio lavoro.

Procedeva il medesimo verso il suo termine allorche stabilitasi in questa Regia Eniversite di Cagliari una caltedra di Storia naturale, si scregliò ad ma tralto non solo nei giovani studenti, ma anche in quelli coi quali essi conferivano, un maraviglioso amore alla scicnza Ornitologica, il quale ben si poti intendere dall'affuenza ogmi di maggiore dei medesimi al heyio Museo onde studiarvi praticamente le scritte teoric, dall'ariditì colla quate raccoglievano le dichiarazioni sui dubbj, rhe mi proponevano, e dalla profonditi delle osservaziomi di confronto, che opponcvano alle mie risposte.

Prendendo questi preludj siccome felice angurio di un bell'arrenire per questo genere discienza tra miei commazionali, rolli contriburvi in quento per me si poleca, e sperai di riusrivi farilitando ai gioxami dilellanti il graduato passaygyio da um stuatio di pura rioreaziome a quello profondo della secienza. 
Con questa cista spinsi il mio lavoro oltre i confui permessi ad un semplice Elcnco, che era quello appunto che stabilivo nel mio primitivo progetto di presentare senza le forme scientifiche, e mi studiai di descrivere distintamente i caratteri dei generi. acemodandomi al metodo del mio prediletto Temmincli nella distribusione di detti generi sotto li rispettivi loro ordini, e delle sperie sollo li relativi loro generi, e usandone pur il frasario sempre che non mi ronsigliò altrimenti qualche particolare regione.

Porta questa mia operuccia il doppio titolo di Elenco degli I'ccelli, che trovansi nell'Isola di Sardegna, od Ornitologia Sarda. Posi il primo, rispettivamente agli scienziati, i quali d’altro non abbisognano per arricchire le loro cognizioni ornitologiche, rhe d'una semplice enumerazione delle sperie, che vi si trovano, coll'aggiunta tuit'al piu dell'indicazione se vi siano permanenti o di passaggio tanto periodico che accidentale: posi laltro in riguado dei giouani connazionali non ancora iniziati nella storia naturale, $i$ quali sentendosi inclinati alle cognizioni ornitologiche abbisognano di alcune nozioni elementari.

Mi sono diffuso nel tracciare i caratteri dei fjeneri, ed enarrure le loro principali abitudini, perche essendo soli 80 quelli compresi nell'Ornitologia Sarda, ho creduto che non risulteri difficile ai giovani studenti il raccomandume alla memoria le descrizioni, per arerle presenti allorchè si roglia riconoscere la famiglia, cui appartenga la sperieche si studia, per classificrarla poscia a suo posto dopo diaterne esuminate le particolariti distimtive non notale espressamente nel genere. 
Se per l'opposto nel descrivere i caratteri spercifici dei nostri Éccelli mi son ristretto ai comnotati li piu distinti, ed apparenti; se mi sono limitato ad abbozzare i soli maschi in perfetla livrea trasandando le femmine, le varietà, e le diverse età di ambi i sessi, cosi mi consigliarono di fare alcune ragioni delle quali noterò queste sole: $1^{\circ}$ Perchè il Manuale d'Ornitologia del Temminck colle parti postume, od appendici, di cui io nella massima parte mi prevalsi, niente lascia a desiderare per uno studio radicale di questo genere, e pertanto risulterebl" superflua ogni ulteriore spiegazione, che avessi voluto aggiungere al mio lavoro.

$2^{\circ}$ Perche destimato essendo questo lavoro per primo erudimento e preparamento ad uno studio piu profondo se io mi fossi allargato nelle distinzioni necessarie per raprpresentare gli ucrelli nei diversi loro sessi e tempi, aurebbe perduto il pregio della brevità, la quale lanto giova per scolpire risentilamente le primarie nozioni d'una sciensa.

$3^{\circ}$ Perche avendo questo mio lavoro doppia destinazione, epperciò anche doppio titolo in fronte, mal si sarebbe conciliata con un Elenco una lunga, e circostanziata leoria sugli articoli che conteneva, qualora mi fossi voluto diffondere d'aveantaggio, siccome ho falto per generi, nella descrizione delle specie.

Nel porgere al pubblico questo mio lavoro, qualunque egli sia, spero che gli scienziati mi saranno indulgenti, cosi sulla preferenza che ho dato al Professore Temmincli nel seguire rigorosamente il sistema degli ordini, divisioni, sezioni. 
generi e specie da hi adollato, come sulle molte imperfesioni che riconosceranmo in questo lacoro: e mi auguro che $i$ giovani miei connazionali, alla di rui istrusione ì diretto. vorranno cortesemente aggradirlo.

Io diro con fianche "sincere parole rio che mi mosse. " mubblicarlo il desiderio di far cosa utile a quelli che voyliono iniziarsi mell Ornitologia della Sardegna, e un servizio ayli scienziali che desiderano conoseere la medesima per aver complesa la Ornitologia Europea. 


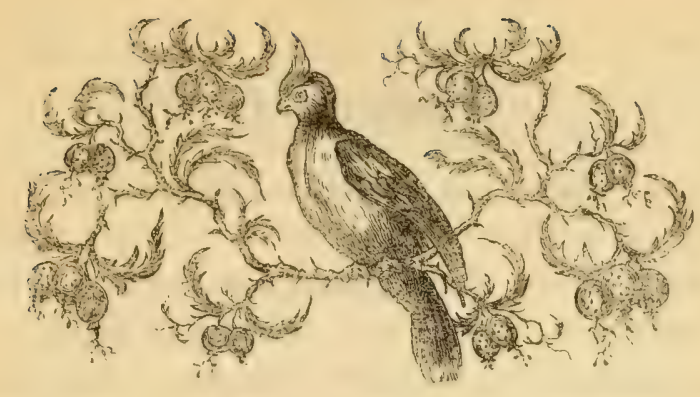

\section{ORNITOLOCGIS SIRII}

\section{ORDINEI.}

\section{RAPACI - RAPACES.}

Becco corto, robusto, compresso ai lati, curvo verso l'estremita, mandibola superiore coperta alla sua base da una cera, narici aperte, piedi di grandezza media, nervosi, forti, piumosi fino alle ginocchia o fino alle dita, e talvolta fino alle unghie: tre dita innanzi, ed uno indietro, articolati nel medesimo lungo, interamente divisi o uniti alla base da una membrana, ruvidi inferiormente, forniti d'unghie grosse, acute, retrattili, e curve.

In quest'ordine si comprendono tanto i diurni che i notturni. Questi ultimi hanno si nell' esterno che nell' interno differenze notabili. 


\section{GENERT}

\section{Avoltojo. - Vultun (Illiger)}

Carattere generico. Becco grosso, duro, molto piu alto che largo, base coperta da una cera: mandibola superiore dritta curvata solamente verso la punta, mandibola inferiore dritta rotondata, el inclinata verso la punta: testa nuda, e coperta da una peluria cortissima : narici nude, laterali, fesse diagonalmente verso gli orli della cera; piedi forti muniti d'unghie debolmente armate: il dito di mezzo lunghissimo unito nella base all' esteriore: ali lunghe, il primo remigio corto piu del sesto, il secondo ed il terzo men lunghi del quarto, che è il piu lungo.

Tutti gli Avoltoj quando sono sazii stentano a prendere if volo, ed in simili circostanze si possono prendere perfino a colpi di bastone. Avidi come sono di carne si scaricano sorra le bestie morte, e cadareri, e non gli abbandonano finchè non gli abbiano spolpati affatto, e con ció prestano segnalato servigio ai viventi liberandoli dalle esalazioni che tramanderebbero le carni putrefatte.

\section{SPECIE I.}

Vultur Auricularis (Dand).

Vautour Oricou.

Contruxiu, e Bentruxiu. Capo Meridionale.

Benturzu, e Antur:u. Capo Settentrionale.

Questo Aroltojo il piu potente del suo genere, ha il beect: robusto, elevato e molto curvato. Si conosce dal collare com- 
posto di piume corte e rotonde, dalle piume del ventre lunghissime, appuntate, curvate che mal ricoprono una peluria di bianco puro, e dalle coscie senza piume, coperte solamente di detta peluria. Comune, stazionario, ha le stesse abitudini della specie seguente, e nidifica nelle fessure delle rocche delle piu alte e scoscese nostre montagne.

\title{
SPECIE II.
}

\author{
Vultur Cinereus (Linneo). \\ Vautour Arrian. \\ Avoltojo Nero, \\ Contruxiu, e Bentruxiu nieddu. C. M. \\ Anturzu, e Benturzu. C. S.
}

Parte superiore della testa, è la nuca spogliata di piume, e guernita d'una peluria d'un bruno giallognolo, la pelle d'un colore azzurrognolo, sulla base del collo s'innalza un mazzo di piume a barbe sciolte: il color generale delle piume ì d'un bruno tendente al nero. Comune, stazionario, abita in tutte le nostre montagne, se ne fa preda in ogni stagione ed in diverse livree; nidifica pure fra noi. Ebbji varie volte dei novelli in peluria, due ne ebbi sul finire del maggio 1837, unitamente alli madre che fu presa nei monti di Capoterra nel proprio nido.

\section{SPEGIE III.}

Vultur Fulvus (Linneo)

Vautour Griffon.

Avoltojo Griffone.

Bentruxiu murru. C. M.

Anturzu o Benturzu. C. S.

La testa ed il collo gunerito di peluria diun bianco sporoto. 
farte inferiore del collo attorniata di molti ranghi di lunghe piume alfilate d'un bianco rossiccio; in mezzo al petto ha uns spazio coperto di color terra d'ombra contornato da peluria bianca : color generale bruno rossiccio. Anche questa specie nidifiea fra noi, ed è similmente comune nei luoghi di montagnas.

\section{SPECIE IV.}

\section{Vultur Kolbii (Dand). \\ V'aulour Chassefiente.}

Bentruxiu C. M.

Anturzu, с Benturะu C. S.

Si distingue facilmente dalla specie precedente dalla forma delle penne delle ali, e delle parti inferiori, poichè rotonde alla punta, mentre quelle del Griffone sono appuntate e lunghe. Colore generale delle piume di caffe - latte chiaro, o isabella; gozzo d'un bruno carico: testa e collo coperto d'una peluria corta.

Questa specie è la più comune in Sardegna, ha le stesse abitudini delle altre precedenti, e nidifica similmente fra noi.

Il genere Avoltojo, Vultur, è sparso in tutto l'antico continente, e pare che manchi nel nuovo.

Nota. Del secondo genere Chataretes Percuopterus non consta d'essersi iu tempo alcuno fatto preda, per cui io credo che manchi nell'isola, tuttoche il Cetti nella sua Storia degli Uecelli di Sartegna pretenda che vi esista. Son piu presto d'avviso che il Perenottero del detto autore sia il Grifone maschio, poichè a questo corrisponde precisamente la dimensione. dat lui datagli di tre piedi e qualche pollice, intantoche il Perenottero nom ha più di due piedi ed un pollice: dico il Grifone maschio, perche fra goli uec vli di rapina sono semple piu piecoli i maschi delle femmine. 


\section{GENERE III. \\ Gipate Gypaectus (Storr)}

Éarattere generico. Becco forte, lungo: mandibola superiore sollevata verso lit punta, che si piega in forma d'uncino verso la medesima. Un mazzetto di peli gli forma una specie di barba solto la mandibola inferiore. Narici ovali ricoperte di peli diretti in avanti. Piedi corti, quattro dita, le tre d'avanti riunite per mezzo d'una corta membrana, quello di mezzo lunghissimo: le unghie poco armate: ali lunghe, il primo remigio un po' pii corto del secondo e terzo, che sono i piis lunghi.

\section{SPEGIE V.}

Gypaetus Barbatus (Cuvier).

Gypaëte Barbu.

Avoltoio Barbato.

Barbudu o Ingurt' Ossu C. M.

Benturzu Barbudu C. S.

T'esta e parte superiore del collo d'un bianco giallicio, parte inferiore del medesimo pelto e ventre d'un rosso arancino, dorso e parte superiore d'un grigio bruno carico, ma ciascuna penna strisciata in bianco longitudinalmente.

E pure stazionario fra noi, ma se ne prende di rado, forse per la gran difficolti di cacciarli, mentre abitano nelle piir alte e scoscese montagne. Ne ho avute in diverse stagioni dell'anno, ed in varie livree anche in quella da giorane.

II genere Gypaeto, Gypactus, si frova solamente nell'antion Continente. 


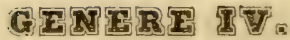

\section{Falco. Falco. (Linneo)}

Carattere generico. Testa coperta di piume, becco ricurvato per lo piu dall'origine: una cera colorita piu o meno coperta di peli alla base : mandibola inferiore obliquamente ritondata : tanto questa che la superiore qualche volta incavala. Narici laterali, rotonde, ed orali forate nella cera; piedi a tarsi coperti di piume, o lisci a scaglie, tre dita d'avanti; l'esteriore d'ordinario unito alla base con quello di mezzo da una membrana. Unghie acciajate ricurvatissime e mobili.

\section{Prima Divisione}

\section{Falconi (propriamente detti)}

I Falconi propriamente detti, che si comprendono in questi divisione, si riconoscono dai seguenti caratteri : mandibola superiore armata d'un dente forte e talvolta di due, che s'impegnano in tacche della mandibola inferiore; hanno inoltre il primo e terzo remigio eguale, il secondo piu corto di tulii. Questi portano il nome d'uccelli da preda nobili, forse per la prerogativa in antico accordata esclusivamente alla nobilta di prevalersi d'essi per la caccia.

\section{SPECIE VI.}

Falco Lanarius (Linneo).

Faucon Lanier.

Falco Lanario.

Stori Perdighinu C. M.

Astore C. S.

Sommiti della testa d'un rosso chiaro, con macrhic obhun- 
ghe e brune: parte superiore del corpo d'un bruno cenerino, colle piume frangiate d'un rosso chiaro: parte inferiore d'un bianco puro a macehie lanceolate di bruno chiaro.

Di passaggio accidentale. Tre soli individui n' ebbi, uno nell'inverno del 1834, l'altro in quello del 1837, ed il terzo ai 15 febbraio del 18\%2. Quest’ultimo individuo era femmina , cacciato sulle sponde dello stagno di Santa Gilla, a otto miglia *irea di distanza sud-est da Cagliari, esiste nel nostro Museo.

\title{
SPECIE VII.
}

Falco Peregrinus (Linneo).

Faucon Pelerin.

Falco Pellegrino.

Astori Perdinghinu C. M.

Astore C. S.

Testa e parte superiore del collo d'un azzurro nericcio, le altre parti superiori d'un azzurro cinerino, con delle striscie d'un colore piu oscuro, gola e petto d'un bianco puro, con picrole striscie longitudinali; le altre parti inferiori di biance sporco. Nidifica fra noi nei luoghi montuosi, e redesi tuto lanno nelle rupi di Sant'Elia nelle vicinanze di Cagliari.

\section{SPECIE VIII.}

\author{
Falco Subbuteo. (Latham). \\ Faucon Hobereau. \\ Falchetlo da Uccelli. \\ Storixeddu C. M. \\ Astoreddu C. S.
}

Gola bianca, con due striscie larghe e nere che discendono lateralmente lungo il collo : parte superiore d'un nero azzurro- 
gnolo con delle bordure chiare, parte inferiore, bianchiceie con delle striscie longitudinali nere.

Di passaggio annuale in autunno.

\section{SPECIE IX.}

Falco Aesalon (Temminck).

Faucon Emirillon.

Falcone Smeriglio.

Storittu C. M.

Astorittu C. S.

Parti superiori del corpo come Ia coda d'un cenerino tendente all'azzurro con delle macchie longitudinali nere in ciascuna piuma : gola bianca: parti inferiori d'un 'giallo rossiccio con delle macchic oblunghe in forma di lagrime.

Più comune del precedente, e d'inverno ne fanno preda $i$ cacciatori delle grive, delle quali pare che ne siegua il corso.

\section{SPECIE $X$.}

Falco Timmunculus (Linneo).

Faucon Creserelle.

Falchetto da Torre o Gheppio.

Tilibricu o Zerpedderi. C. M.

Tilibriu C. S.

Sommiti della testa d'un grigio azzurrognolo: parti superiori d'un bruno rossiccio regolarmente seminato di macchie angolari nere: parti inferiori d'un color rossiccio con delle macehie oblunghe e brune.

Comunissimo stazionario, nilifica nelle vechice torri, a negrli antichi fabbricati, ed is conosciuto da fulli. 


\section{SPECIE XI.}

Falco Timnunculoides (Natheri).

Faucon Cresserellette.

Falchetto Cresserelletta.

Tilibricu o Zerpedderi C. M.

Tilibricu C. S.

Sommita della testa, parti laterali del collo, e nuca d'un cenerino chiaro senza macchie, dorso, scapolari, e la copertura delle ali d'un rossiccio oscuro senza alcuna macchia; gola chiara, le parti inferiori d'un rossiccio chiaro, seminato di piccole mac:chie, e di striscie longitudinali nere.

Marissima è questa specie fra noi: un solo individun potci ('saminare nel maggio del 1836, ma non fu possibile prepararto a causa del cattivo stato in cui trovavasi.

\section{SPECIE XII.}

Faleo Rufipes (Bechst).

Faucon a pied rouges ou Kobez.

Falchetto Barletta.

Storixeddu C. M.

Astoritlu C. S.

La testa, il collo, petto, ventre, e generalmente tutte le parti superiori di color grigio di piombo senza alcuna macchia; le coscie, il basso-rentre, e le coperture inferiori della coda di un bel rosso carico; la cera, il contorno degli occhi, ed i piedi d'un rosso cremisi.

Di passaggio in primavera. Ne fu predato uno in perfella livrea nelle vicinanze d'Iglesias nell'aprile del $\mathbf{1 8 3 9}$ dai signori Ingresi lord Louvaine ed Enrien Ipton in una eseursione di 
(accia. Questo Falchetto ama a prelerenza di vivere in luoghi paludosi. Trovai sempre il ventricolo di quest'uccello pieno di frammenti d'insetti, particolarmente dell'ordine dei Coliopteri.

\section{SPECIE XIII.}

Falco Eleonora (Gènè).

Faucon Eléonore.

Falco della Eleonora.

Stori o Zerpedderi.

Rassomiglia molto alla specie terza (Subuteo) ma diversilica dalla medesima per la più grossa taglia e per il colore delli cera azzurrognolo.

Questa nuova specie fu scoperta dal chiarissimo signor generale cavaliere Alberto Della-Marmora negli Isolotti del Toro e la Vacea, vicini a quella di San Pietro ove nidificano, e vi si vedono stazionarj. Fu pubblicata dal signor professore Giuseppe Genè cavaliere del Merito Civile, e direttore del Regio Museo di Torino nelle Memorie della Reale Accademia delle Scienze, serie $11^{\mathrm{a}}$, tomo $11^{\circ}$, pag. 4.

\section{Gecondia Divishome}

AQuiLE (propriamente dette).

Le aquile hanno il becco assai lungo, che non si curva immediatamente alla base: piedi robusti e nervosi, ditit simili ed armati d'unghie possenti e areuatissime: le ali lunghe. primo, secondo e terzo remigio i piu lunghi.

Questi uccelli furono in tutti i tempi con ragione considerali come i crudeli dominatori dell'aria ; sono feroci, e d'una forza straordinaria : vivono solitarii per soddisfare il vorace loro appetito nelle piu seoscese rupi. Sono avidi di strage, e divo- 
rano la carne palpitante. Alcune specie fanno ugualmente uso di pesci. Tutte generalmente hanno la vista cosi acuta, che dal piu alto dell'aria vedono e distinguono il rettile strisciante sulla superficic della terra, e piombano sopra di lui come una saetta.

\section{SPECIE XIV.}

Falco Imperialis (Temminch).

Aigle Impérial.

Aquila Imperiale.

Achili o Achiloni C. M.

Abila C. S.

Sommita della testa e nuca guernite di piume rossiccie bordate di rosso vivo: le parti inferiori d'un bruno nero carico, at? eccezione del basso ventre che ha un rosso gialliccio, parti superiori d'un bruno opaco.

Di raro si fa preda di questa specie, ma è positivo che nidifica sulle nostre montagne, poichè ne fa predato un individuo femina in peluria da giovane, e dopo di averlo il sig. Teologo D. Giuseppe Gambella Rettore d'Itiri, allevato in cusa, graziosamente ne fece dono al nostro Museo, ove esiste tuttora.

\section{SPECIE XV.}

Falco Fulvus (Linneo).

Aigle Royal.

Aquila Reale.

Achili o Achiloni C. M.

Abila C. S.

Sommita della testa e nuca coperta di piume di color rosso vivo dorato: tutie le altre parti del corpo d'un bruno oscuro più o meno nero, secondo l'eta.

Nidifica pure fra noi, ed ì comme in tutta l'isolit, 


\section{SPECIE XVI.}

Falco Albicilla (Latham).

Aigle Pygarghe.

Aquila di mare o Pigarga.

Achiliera. C. II.

dbila. C. S.

Testa e parti superiori del collo d'un cenerino bruno abbatstanza chiaro: tutte le piume del corpo e delle ali d'un bruno sporco o cenerino, senz'alcuna macchia, coda bianca.

Frequenta le sponde dei nostri mari. Un magnifico individuo ne ebbi in perfelta livrea preso nel maggio 18.1, ed esiste nul nostro Museo.

\section{SPECIE XVII.}

Falco Bonelli (Temminck)

Aigle Bonelli.

Aquila Bonelli.

Achiloneddu. C. M.

Abilastru. C. S.

Parti superiori d'un bruno piì o meno opaco secondo l'eti piii adulta : dorso e parti superiori delle ali d'un bruno nericeio bordate d'un bruno rossiccio; grandi coperture delle ali, e sca polari d'un bruno nericcio, gola, pelto, fianchi, ventre e basso ventre d'un bianco puro prendendo in qualche parte una leggiera linta isabella; lutte queste piume sono macchiate in forma di lacrime di color brtino carico.

Comunissima fra noi, nidifica sulle lessure delle rocche piu elevale. Ne ebbi diverse volte varj individui in peluria presi alla rollina di fant'Elia in vicinanza di Cagliari, qualche volli anrhe colla madre: se ne prendono peró di raro in perfelta livea. 


\title{
SPECIE XVIII.
}

Falco Brachydactilus.

Aigle Jean le Blane

Falco terzo d'Acquila.

Tesla grossissima, sopra gli occhi uno spazio guernito di peluria bianca: sommita della testa, gola, petto e rentre bianca, variate da macchic rare d'un bruno chiaro, manto, e copertura delle ali di color bruno, origine delle piume bianco puro.

Rarissima comparisce fra noi questa specie : un solo individuo ne fu predato in autunno, ed esisté nel nostro Museo.

\section{SPECIE XIX.}

\author{
Falco IIaliacus (Linneo). \\ Aigle Balbusard. \\ Aquila Pescatora. \\ Achili de Pisci C. M. \\ Abila Marina. C. S.
}

Sommiti della testa e nuca guarniti di piume sfilate nere bordate di bianco gialliccio, quelle delia nuea lunghissime: parti superiori brune, inferiori bianche con due striscie di color bruno carico lateralmente lungo il collo.

Arriva in autunno, e parte in primavera, abita in riva dei nostri mari e negli stagni, ed è conosciulissimo da lutli i nostri pescatori rhe sorente la prentono coll'amo. 


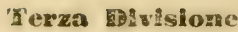

\section{Astoni}

Questa divisione si distingue per il tarso molto elevato e piu lungo del dito di mezzo.

Ali corte, primo remigio molto più corto del secondo, il terzo quasi eguale al quarto ch' è il piủ lungo di tutti.

\section{SPECIE XX.}

Falco Palumbarius (Linneo).

L'Autour.

Astore

Stori Colombinu C. M.

Astore C. S.

Le parti superiori d'un cenerino azzurro, le parti inferiori hanno sopra un fondo bianco dei tratti transtersali, e delle striscic strette longitudinali d'un bruno carico.

Di passaggio periodico annuale in autunno: frequenta di preferenza i luoghi boscosi.

\section{SPECIE XXI.}

Falco Nisus (Linneo).

L'Espervier.

Falchetto Fringuellajo.

Feridori. C. II.

Astorittu Feridore. C. S.

Parti superiori d'un cenerino azzurrognolo con una macilia bianca alla nuca, parti inferiori bianche con dei tralli longifuAinali sotto la gola, e transversali nelle parti inferiori. 
Arriva in autunno, e se ne fa preda in quantiti in inveruc dagli uccellatori, preferisce i luoghi montuosi, ove fa gran distruzione degli uccelletti che vi abitano.

\section{Quarta bivisione \\ MILA I I O MIL VI}

Hanno questi le ali lunghe, primo remigio cortissimo, il terzo o il quarto piu lungo di tulti. Tarsi sottilissimi, il loro rolo è tanto elegante, che sembrano nuotare nell'aria facendo circoli senza muovere punto le ali.

\section{SPECIE XXII.}

Falco Milvus (Linneo).

Milan royal.

Nibbio

Zuaddia. C. M.

Atturolia e Tirulia. C. S

Parti superiori d'un bruno rosso con le piume bordate d'un color più chiaro: parti inferiori d'un rosso di rugine variato da liste longitudinali brune, le piume della testa e del collo lunghe biancastre, rigate longitudinalmente in bruno : coda forcuta.

Fa slazione, e nidifica fra noi, pone il suo nido nei pioppi. In tutti i villaggi temono quest'uccello che volteggia nelle corti, e si vede ad un tratto slanciarsi sopra le galline, e portarseli in aria.

\section{SPECIE XXIII.}

Falco Ater (Linneo).

Milan noir ou Elolien.

Nibbio.

Zuaddia. C. B.

Atturolia. C. S.

Parti superiori d'un grigio bruno carico, inferiori d'un brums 
rossiccio con delle macelie fongitudinali nel centro delle piume, testa o gola a righe longitudinali bianchicerie e brune. Codia pochissimo forcuta.

Meno comune del precedente e non vi è differenza aii nome.

\title{
Quinta Divisione
}

Lanieri o Pojaxe

Questi si distinguono dai tarsi corti, e le coscie fortemente piumate, i quattro primi remigi intaccali, il primo cortissimo, il secondo ed il terzo meno lunghi del quarto che supera tulti gli altri.

\section{SPECIE XXIV.}

\author{
Falco Buteo (Linneo). \\ La Buse. \\ Falco Capone. \\ Stori C. M. \\ Astoreddu C. S.
}

Parti superiori, collo e petto d'un bruno carico, gola e ventre? d'un grigio bruno, ma variato da macchie brune opache.

Durante l'inverno si fa caccia di questi falchi, i quali sembrano soggetti a variare non solamente nella dimensione, ma nel colore delle piume, e dell' iride, avendone avuto molti vivi aveano aleuni l'iride di color castagno chiaro, altri di bianco sporeo, ed altri d'un bellissimo giallo. 
$-17-$

SPECIE XXV.

Falco Lagopus (Linneo).

Buse Pallue.

Falco Calzato.

Stori C. MI.

Astore C. S.

Testa, parte superiore del collo, gola, petto, e coscie d'un bianco gialliccio variato da larghe striscie oblunghe brune: manto, copertura delle ali e del dorso d'un bruno nericcio : le altre piume di giallo rossiccio, piedi impiumati quasi fino alle dita .

Di passaggio accidentale. Un solo individuo predato ad Iglesias nell'inrerno del 18.3 4 esiste nel nostro Museo.

\section{Sesta bivisime \\ Nimio o Circi.}

Hanno questi i piedi a tarsi lunghissimi, e sottili. Il corpo snello: la coda lunga e rotonda: ali lunghe, la prima penna remigia molto corta, meno lunga della quinta: la seconda un poro pii corla della quarta: la terza e la quarta le piu lunghe.

\section{SPECIE XXVI.}

Falco Rufus (Linneo).

Busard Harpaye ou de Marais.

Falco di Palude.

Stori de Pisci C. M.

Astore Marinu C. S.

Testa, collo e petto d'un bianco gialliccio con numerose marrhie longitudinali brune nel centro di ciascuna piuma : gli sca- 
polari, e copertura delle ali d'un bruno rossiccio: ventre, fianchì, coscie, ed il basso ventre d'un rosso di rugine con qualche macchia gialliccia.

Stazionario fra noi vedesi tutto l'anno swolazzando attorno ai nostri stagni, e nelle sponde del mare, e particolarmente nel luogo denominato la $S c a f f a$ : è soggettissimo a variare di livrea: il nostro $\mathbf{R}^{0}$ Museo ne possiede in varie livree: nidifica fra noi: fa il suo nido nei cespugli in ricinanza al mare.

\section{SPEGIE XXVII.}

Falco Cyaneus.

Busard Saint Martin.

Albanella.

Storixeddu.

Astorillu.

Testa, collo, dorso, ali, e groppone d'un grigio azzurrognolo, ventre, fianchi, e basso ventre d'un bianco puro senza macchie.

Di raro si fa preda di quest' uccello. Due individui furono colti a Pula, uno in livrea da giovine, e l'altro di adulto. Entrambi sono deposti nel $\mathbf{R}^{0}$ Museo.

\section{SPECIE XXVIII.}

Falco Cineraceus (Montagu).

Busard Montagu.

Albanella.

Tutte le parti superiori d'un cenerino azzurrognolo opaco: due striscie nere transversali sulle penne secondarie delle ali : gola e petto d'un renerino azaurngnolo chiarn: ventre, coscie. 
liamehi e basso ventre hianchi, variati da striscie longiludinali d'un bel rosso.

Un solo individuo ne fu cacciato in autumno in livera dia gionvane. Il genere Falcone, Falco, is sparso tanto nell'antico che nel nuovo continente.

\section{GENTRE W. \\ Civetta - Strix (Limeo).}

Carattere generico. Becco curvo schiacciato, colla base circondata da una cera che è coperta da peli rigidi, o penne setace dirette in avanti : narici forate lateralmente sul bordo interno della cera, rotonde, aperte, nascoste in tutlo od in parte sotto i peli : testa voluminosa; occhi grandissimi posti in ampie orbite guernite di penne rigide: una membrana socchiudente: orecchie grandi, bocea sommamente fessa : collo brevissimo : piedi semplicemente coperti di piume, spesso sino alle unghie: tre dita d'avanti ed uno di dietro interamente divisi : l'esterno reversibile: ali alquanto appuntite coi remigi primarj dentellati al bordo esterno: primo remigio piu corto di tutti : secondo che non giunge all'estremita del terzo che di tutti è il più corto.

Questo genere componesi di specie che hanno tulti un'aria di famiglia.

Per i grandi servigi che questi uccelli prestano all'agricoltura distruggendo i ratti, sorci, e topi campagnoli, la loro vita dovrebbe essere piu rispettata, ma al contrario per qualche pregiudizio che tuttora sussiste fra gl' idioti è sempre insidiata.

Tota. La prima divisione delle Civette nropriamente dette manca affatto fia noi, e son quelle appunto che Temminch nomiua nella prima sezione Auripitrini, o diurne, perchr verluno glusste hen distintamente, e cacciann la loro preda in pieno gioino. 


\section{Siecollon Sizionte.}

BARBAGIANI NOTTURN.

Questi cacciano al crepusculo e si naseondono appena fatlo giorno.

\section{SPECIE XXIX.}

Strix Flammea (Linneo).

Chouette Efraic.

Strige ed anche Allocco.

Stria o Istria C. M.

Strea e Istria C. S.

Parti superiori d'un gialliccio chiaro variato da righe grigie e brune in forma di zigzag, e seminato d'una moltitudine di piccoli punti bianchicci : faccia e gola bianca : parti inferiori in alcuni individui d'un bianco rossiccio seminato di piccoli punti bruni, in altri d'un bianco risplendente macchiato di piccoli punti brunastri, in altri finalmente senza la menoma apparenza di macchie.

Comunissimo e stazionario, nidifica nei vechi edifizi e nelle muraglie, vedesi, e si fa sentire la sua vore lutto l'amuo sul nostro terrapieno.

\section{SPECIE XXX.}

Strix Passerina (Autorum).

Chouette Cheviche.

Civella.

Cuccumen C. 11.

Cuccumiau C. S.

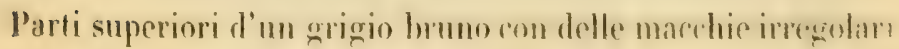


bianche: il petto d'un bianco puro: le altre parti inferiori d'un bianco rossiceio con delle macchie d'un bruno cenerino.

Nidilica pure fra noi nei rechi alberi, e nei telli delle case di campagna.

\section{Seconda Divisione}

Queste si distinguono per lue mazzelti di piume che hanno prazzate sulla testa a guisa di corna, le quali rialzano e abbassano a loro piacimento.

\section{SPECIE XXXI.}

Strix Brachiolos (Latham).

Hibou Brachiole.

Allocco.

Stria C. M.

Strea C. S.

Due o tre piume corte formano sulla fronte delle corna poco apparenti: testa piccola: le piume delle parti superiori di color bruno nericcio contornate d'un giallo di terra : parti inferiori di colore isabella, con delle macchie longitudinali d'un bruno nericcio.

Yon è molto comune, e non so se nidilichi fra noi

\section{SPECIE XXXII.}

Strix Otus (Linneo).

Hibou Moyen Duc.

Allocco.

Stria C. M.

Strea C. S.

Dieci piume nere bordate di color giallo di terra e bianchic- 
cio formano le corna: parti superiori d'un rosso giallicecio cons macchie irregolari di bruno carico, e grigio cenerino : parti inferiori d'un giallo di terra chiaro con delle macchie oblunghe d'un bruno nericcio.

Di raro si fa preda di questa specie. I due bellissimi individui che possiede il nostro Museo furono predati dai signori dilettanti di caccia Francesco Marghinelti e Francesco Giuitta, ai quali sono debitore di altre specie d'uccelli che graziosamente ebbero la compiacenza di offrirmi.

\section{SPEGIE XXXII.}

Strix Scops (Linneo),

Hibou Scops.

Assiolo.

Zonca C. M.

Tonca C. S.

Le corna formate da piccole piume riunite a mazzetto, tanto queste che quelle della testa di color bruno marcato con piccoli punti neri. Il rimanente delle piume superiori d'un cenerino tendente al rosso: parti inferiori di color meno opaco.

Comune stazionario, nidifica nelle fessure delle rocehe e nei tronchi degli alberi.

II genere Civelta Strix si trova nell'antico e nuovo Continente.

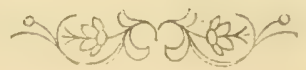




\section{$-23-$ \\ ORDINE II.}

\section{rongs. \\ OMNIVORI - OMNIVORES.}

Becco mediocre, robusto, lagliente ai bordi: mandibole superiori piu o meno ricurvate verso la punta. Quattro dita, tre davanti, e uno indietro: ali mediocri : remigj terminati in punta : il secondo ed il terzo piu corti del quarto.

\section{GENDRE W \\ Convo-Convus (Limeo).}

Carattere generico. Becco dritto, grosso, compresso ai lati, urvato verso la punta, bordi taglienti : narici basali aperte e nascoste da peli diretti in avanti: piedi con tre dita davanti ed uno di dietro quasi intieramente divisi : tarso piu lungo che il dito di mezzo: ali appuntate: il primo remigio di mediocre lunghezza, il secondo ed il terzo piu corti del quarto che è il piu lungo.

Il danno che questi uccelli fanno all'agricoltura è ben compensato dal servizio che prestano di purgare la terra dalle larve degli insetti, e delle locuste. In alcuni luoghi sono considerati come benefattori, e quindi sono rispeltati. 
Convi (propriamente delli).

SPECIE XXXIV.

Corvus Corax (Limeo).

Corbean Noir.

Corvo Imperiale.

Crobu Nieddu C. II.

Corbu e Corvu C. S.

Di un bel nero lucido a rillessi porporini.

Di passaggio annuale al principiar dell'imerno, c parte in primavera, ma ne rimangono sorenti alcuni che si vedones a copie. Abita le montagne.

\section{SPECIE XXXV.}

Corvus Corone (Linneo).

Corneille Noir.

Cornacchia Reale.

Crobu Nieddu C. M.

Corbu neru e Corvu C.S.

D'un nero piu oscuro del precedente a riflessi color violetto. Si distingue dall'altro nella grandezza, essendo minore d'un quarto, e dall' iride color di nocciuola, mentre l'altro l'ha doppia, cioè grigio bianco, e cenerino bruno.

Comunissimo fra noi vedonsi a torme nelle pianure, particolarmente in vicinanza del mare.

\section{SPEGIE XXXVI.}

Corvus Cornix (Linneo).

Corneille Mantelée.

Cornacchia mantellata o Mulacchia.

Carroga Braxia C. M.

Carroga Barza, Corrionca, e Corronca C. S.

II collo e futto il corpo d'un prigion renerino, gola. ali " 


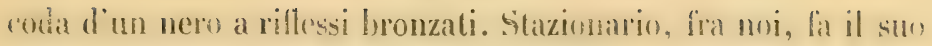
nido nei pioppi.

\section{SPECIE XXXVII.}

Corvus Frugilegus (Linneo).

Freux.

Corvo nero.

Crobu C. M.

Corroga niedda C. S.

Base del beceo, narici, gola, e d'avanti della lesta spogliari di piume. Colore generale nero a rillessi risplendenti di porpora e violello. Comune durante l'inverno.

\section{SPECIE XXXVIII.}

Corvus Monedula (Linneo).

Choucas.

Cornachiella.

Carroga C. M.

Tacula C. S.

Sommita della testa d'un nero cangriante in violetto, nuca e parti suneriori del collo d'un grimio cenerino: lutte lo altre parti superiori d'un nero lustro di violelto : le inferiorid un nero opaco.

Ora comune ma non piu comunissinss come negli anni addietro che si vedera sopra i fetti di tutte le case anche dentro di cilta, il permanente alloggio che oggidi hanno ir liantico catstello di San Michele.

Il grenere Corvo Corvus è sparso da prer fulto. 


\section{GENRIEII. \\ Garrule - Garrulus (Brisson).}

Questo genere d'uccelli ha presso a poco le stesse abitudini " lo stesso carattere dei corvi, e hanno la coda molto lunga, e sorente conica.

Sutt. La prima divisione di questo genere, che comprende le piche prupriamente dette, manca fra noi.

\section{Geconda Bivisione}

Gazze.

\section{SPECIE XXXIX.}

Garrulus Gilandarius (Linneo).

Geai Glandivore.

Ghiandaja.

Piga C. M.

Piga C. S.

Testa a ciuffetto, mostacchi neri al becco: fondo delle piume J'un cenerino rossiccio, sulla piega dell'ala due ordini di piume azzurre transversalmente rigate in nero.

Nidifica fra noi sugli alberi, ed è abbondantissimo particolarmente nel Capo settentrionale.

II genere Gazza, Carrolus. Nell'antico e nuovo Continente.

\section{GENER VII]}

\section{Spezza Noce. Nucifraga (Brisson).}

Questo genere è dell'ordine degli Onnivori. Caratteri. Becon grosso, lungo, rilto, convesso alla parte superiore, compresso 
ai lati affilato alla punta: mandibola superiore ritondata, senzal lisca sporgente piu lunga dell'inferiore : narici collocati alla base del beeco, picrole, aperte, celate da peli diretti all'innanzi : quattro dita ai piedi, tre imnanzi e uno dietro, l'esterno saldato alla sua base, quello di mezzo men lungo del tarso: ali uniformi, la prima e la sesta penna remigia eguali, la seconda e la terza piu corta della quarta dhe è la piu lunga. Una sola specie compone questo genere che c̀ la seguente.

\title{
SPEGIE XI.
}

\author{
Nucifraga Caryocatactes (Brisson). \\ Casse-Noix. \\ Nocciolaja.
}

Tutte le piume color di fuligine con grandi machie bianche in forma di lacrime sul dorso: parti inferiori variate da molto hianco disposto longitudinalmente sopra ciascuna piuma.

Accidentale è il suo passaggio, un individuo ne fu predato nelle montagne d'Arizzo in tempo d'inverno, ed esiste nel Regio Huseo; questo grenere trovasi solamente in Europa.

\section{GINRE RX.}

\section{Pirnoconaccia Pyrroconax (Cuvier).}

Carattere generico. Becco mediocre, un poco gracile, più o meno arcuato, tagliente, compresso: narici laterali alla base, ovali, aperte intieramente, nascoste da peli diretli innanzi. Piedi forli, mhusti: tarso piu hugo del dilo di mezzo, quatlon dita 
quasi fotalmente separate, unghe forti ed arcuate: i tre primi remigg sradatamente lunghi, il quarto ed il quinto pii lunghi.

\section{SPECIE XLI.}

Pyrrocorax Graculus (Temminck).

Chocerd.

Corvo Corallino o Gracchio.

Corroga C.S.

Tutte le piume nere a rillessi verdi, violetli e porporini, becen opiedi di colore vermiglio.

Permanemte al monte Gemargentu, e comparisee anche nelle pianure di Sardara e Arcidanu.

Sono debitore di questa notizia all'inlaticabile Generale Alberto della Marmora.

Verso la fine di gennajo $18 \% 2$ un individuo di questa specio venne predato in un olivetto a piccola distanza da Cagliari.

Il genere Pirrocorace, Pyrrocorax. Antico Continente.

Nolu. Il genere decimo Bombycivoca manca tra noi.

\section{GENERE XI:}

\section{Coraccia Comaccias (Limeo).}

C'aruttere generico. Becen mediocre, compresso, più alto che largo, dritto ( tayliente: mandibola superiore curvata verso la punta: narici alla base del bece poste quasi diagonalmente fermate a meti da ina membrana guernita di piume. Piedi a tarso piii sorto del dito di mezzo: Ire dita d'avanti, ed uno di dietro 


\section{- 29-}

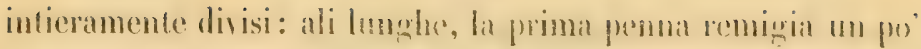
piu corla della seconda che è piì lunga.

\section{SPLCIE XIII.}

Coracias Garrula (Linneo).

Rollier Vulgaire.

Serenone.

Sommiti della testa ed estremiti superiore del collo diun a\%zurro chiaro a rillessi verdi, dorso e scapolari di color rossierio: piccole coperture delle ali d'un risplendentissimo azzurro violetto, parti inferiori d'un azzurro d’acqua masina piic o meno carico.

Di passaggio annuale in primavera ma in pochissimo numero. Abita di preferenza nei hoghi vicini al mare, lo trovai varie volte tra la Maddalena e Ori : piu comuni peró a Oristano.

Il genere Coraccia, Comecias. Antion Comtinentl.

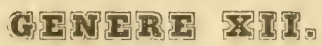 \\ Rigogoro Oriolus (Temmincle).}

Carattere generico. Becco a cono allungato, compressio orizzontalmente alla base, lagliente: mandibola superiore rilevata da una lisca incavata alla punta. Narici aila base del becen forate orizzontalmente, socchiuse da una membrana: piedi arenti tre dita daranti e uno di dictro, tarso piu corto o della lunghezza del dito di mezzo: l'esteriore saldato a quest'ullimo: ali melionri, remigj enti, il terzo piiu lıngo di lulli. 


\section{SPECIE XLIII.}

Oriolus Galbula (Linneo).

Loriot.

Rigogolo.

Canarin Selvaticu C. M.

fanariu Areste C.S.

Cieneralmente d'un giallo d'oro, ali e coda nera, questa terminata in giallo.

Comparisce fra noi in primavera, nidifica e parte primcipiando l'estate: i giorani sono comunissimi, e rari gli adulti in perfetta livrea. Frequentano moltissimo la parte di Pula, e Orri nella stagione suindicata. Il genere Rigogolo Oriolus. Antico Continente ed oceanico.

\section{GENERE XIIT. \\ Storvo Sturnus (Limeo).}

Carattere generico. Becco mediocre, dritto. lungo, conico, compresso, alquanto ottuso: la base della mandibola s' avanza sulla fronte, la punta molto depressa senza incavo, narici alla base poste lateralmente, fermate a meta da una membrana vnota; piedi tre dita d'avanti, ed uno di dietro: il dito esteriore saldato alla base di quello di mezzo: ali lunghe, primo remigio rortissimn, il serondo ed il terzo sonno i piu lunghi. 


\section{SPECIE XLIV.}

Sturnus Vulgaris (Linneo).

Etourneau Vulgaire.

Storno o Stornello.

Sturru Pintu C. MI.

Sturme Pichettadu C. S.

Piume generalmente nericcie con rillessi risplendentissimi di porpora, e verde dorato : parti superiori marcate di piscolissimi punti triangolari d'un bianco rossiccio.

Comunissimo. Arrivano ai primi d'agosto in gran numero, e partono in primavera.

\section{SPECIE XLV.}

Sturmus Unicolor (Della Marmora).

Etourneau unicolore.

Stornello nero.

Sturru Nieddu C. M.

Sturru Neru C. S.

Tutte le piume d'un nero lustro a leggieri rillessi porporini poco risplendenti alle parti superiori, e d'un nero matto alle parti inferiori.

Indigeno, si vedono in grandissimo numero sopra i tetli delle case rustiche di tutti i nostri villaggi, ove si ricoverano la sera per passarvi la notte.

Depongono il loro nido nel luogo suddelto e nelle fessure idelle rocche.

II genere Stornello, Sturnus. Afrien er Asia. 


\section{GENERE XIV}

\section{Torno Roseo Pastor (Temmincli).}

C'arallere yeneriro. Becco coniro alquanto; mandibole compressissine a bordi laglienti con la base nuda, lia superiore debolmente intaceata alla punta che è un po' piegata: narici ovoides poste ai lati del becco, e presso la base, in parte coperta da una membrana pinmata: quattio dita, tre d'avanti dei quali quello di mezzo meno lungo del tarso, si trova saldato al naseere dell'esterno: primo remigin quasi nullo, il secondo ed il terzo piu lunghi.

\section{SPEGIE XLVI.}

Pastor Roseus (Temminck).

Martin Roselin.

Martino Roseo, o Merlo color di rosa.

Testa capellata, collo, e l'allo netlo d'un sero a riflessi violetti: ventre, basso rentre, e dotso d'un bel roseo, ali a coudi d'un bel vinlefto a riflessi, la copertura delle ali ricamata di roseo chiaro.

Accidentale ì il suo passaggio : due individui sono stati predati in perfella livera, e vivissimo is il color roseo. Trovansi deposti nel nostro Museo.

Il genere Dartino, Fastor, delle parti calde dull antion Continente. Di passaggion mel mezoli dell Ėurepa. 


\section{ORDINE III.}

\section{$-0.3060-0-$ \\ INSET TIVORI - INSECTIVORES}

Becco corto, o mediocre, dritto, ritondato, poco tagliente, e al lesina: mandibola superiore curva, incavata verso l'estremita, per lo piu guernita alla sua base di pochi peli ruvidi rivolti rerso la punta. Tre dita anteriori, uno posteriore articolati sulla stessa pianta, l'esterno saldato alla base o unito al medio fino alla prima articolazione.

\section{GENERE XY.}

\section{Vefie Lanius (Limneo).}

Carattere generico. Becco robusto, compressissimo, di mediocre lunghezza, dritto sin dall'origine, curvo soltanto verso la punta, dore formasi un uncinelto, alla base guernito di peli ruvidi diretti per d'avanti: mandibola inferiore dritta, narici poste ad ambi i lati del becco, e presso la sua base, rotonde, per metà chiuse da una membrana a volta che sovente i peli ricuoprono: qualtro dita intieramente divise; tre d'avanti dei quali il medio è meno lungo del tarso: primo remigio di mezzana lunghezza, il secondo un po' piu corto ilel terzo e del quarto che sono i piu lunghi di tulti. 


\title{
SPECIE XLVII.
}

\author{
Lanius Excubitor (Linneo). \\ Pie - Grièche grise. \\ Velia grossa. \\ Passariargia C. M. \\ Monteddada C. S.
}

La testa, la nuea, ed il dorso d'un bel cenerino chiaro: parti inferiori d'un bianco puro; ali corte, nere.

Accidentale ne è il suo passaggio: un individuo ne fu predato in primavera, ed esiste nel nostro Museo.

\section{SPECIE XLVIII.}

Lanius Meridionalis (Temminck).

Pie Grièche Méridionale.

Averla Maggiore.

Passariargia C. M.

Monteddada C. S.

La testa, la nuca, il manto, ed il dorso di colore cenerino oscuro, gola d'un bianco vinato, tutte le altre parti inferiori d'un colore di rino cenerino. Di passaggio come sopra.

\section{SPECIE XLIX.}

Lanius Minor (Linneo).

Pie - Grièche a poitrine rose.

Velia Cenerina o Castorchia.

Passariargia C. M.

Monteddada C. S.

Fronte nera, nuca e dorso cenerino, gela bianca, ali nere, petto e fianchi rosei.

Di passaggio annuale principiando la primavera. 


\section{SPECIE L.}

Lanius Rutilus (Brisson).

Pie-Grieche Rousse.

Averla Capirocca.

Passarargia C. M.

Monteddada C. S.

Fronte nera, sommita della testit, enuca d'un rosso ardente, alto del dorso e ali nere, tutte le parti inferiori d'un bianco puro, comunissimo nidilica fra noi.

\section{SPECIE LI.}

Lanius Colluris (Brisson).

Pie-Grièche Écorcheur.

Averla piccola.

Passariargia C. M.

Monteddada C. S.

Sommila della testa, nuca, alto del dorso d'un cenerino izzurrognolo, manto e copertura delle ali d'un rosso castagno, gola e basso ventre d'un bianco puro, fianchi e ventre d'un rosso roseo, ali nericcie hordate di rosso oscuro.

Arrivano in primavera, e partono al principiar dell'inverno, di rado ne rimane qualcheduno.

Il genere Velie Lanius, eccetlo l'Ameriea Meridionale, si trova dappertutto.

\section{GENERE XVI}

\section{Pigla mosche. Mucsicapa (Limneo).}

Carattere generico. Becco mediocre angolare, piu o meno largo, depresso alla basice, compresso verso la punta che es forte. 
dura, curva, ed intaceatissima, base guernita di peli lunghi e ruvidi : narici collocate a ciascun lato del beceo, e presso la sua base, ovoidi, coperti in parte da alcuni peli direlti in avanti, tarso un po' piu lungo, o lungo come il dito medio: quattro dita, tre d'avanti, i laterali eguali in lunghezza, l'esteriore saldato alla sua base all'esterno: le dita di dietro armate d'un' unghia arcuatissima : primo remigio cortissimo, il secondo meno lungo del terzo e del quarto, che superano gli altri.

Questi uccelli che provredono alla loro sussistenza colla distruzione delle numerose colonie d'insetti che popolano l'aria, si rendono utili all'uomo, liberandolo da quegli sciami incomodi che gli renderebbero insalubre l'aria respirabile.

\section{SPECIE LII.}

Muscicapa Grisola ( Linneo).

Gobe Mouche gris.

Piglia Mosche grigio.

Studa candelas o Papa limu C. M.

Bicca figu C. S.

Tutte le parti smperiori d'un bruno cenerino, fronte tendente al bianco. Sulle piume della testa una riga longitudinale d'un bruno opaco, gola, e centro del ventre bianco : parti laterali del collo, petto, e fianchi seminati da macchie longitudinali d'un bruno oscuro.

Nidifica fra noi nei cortili, e negli antichi edifizj. Arriva in primavera e parte in autunno.

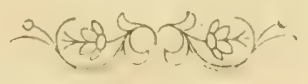




\section{SPECIE LIII.}

Muscicapa Albicollis (Temminck).

Gobe Mouche à collier.

Piglia Mosche a collare bianco.

Bicca Figu C. II.

Bicea Figu C. S.

Sommita della testa, guancie, dorso, piccole coperture delle ali, e tutte le penne della coda d'un nero profondo, la fronte, una larga collana sotto la nuca, e tutte le parti inferiori d'un bianco puro: uno specchio sull'origine dei remigj, e le grandi coperture delle ali bianche.

Comparisce fra noi raramente, e solo in primavera.

\section{SPECIE LIV.}

\section{Muscicapa Luctuosa (Temminck).}

Gobe Mouche Bec Figue.

Piglia Mosche.

Bicca Figu C. M.

Bicca Fiqu C. S.

Tutte le parti superiori del corpo, e le penne della coda nere sscure: la fronte e tutte le parti inferiori d'un bianco puro; le ali anche nere, hanno le penne medie e le grandi coperture bianche, ma queste ultime terminanti in nero nelle loro barbe interne.

Di passaggio qualche volta in aprile, ma di raro.

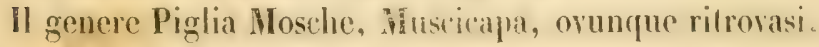




\section{GENIRI XUII.}

\section{Meniro. Tun ous (Limeo).}

Caraltere generico. Beco mediocre, lagliente, intaccalo, curvo e compresso alla punta, dei peli isolati alla sua apertura, narici poste ai lali della sua base, ovoidi mezzo chiuse da una membrank nuda, piedi un po' gracili, tarso piủ lungo del dito intermedio; qualtro dita, tre d'avanti, l'esterno unito per la base all'intermedio, uno di dietro: primo remigio quasi nullo o di lunghezza mezzana, il terzo o talvolta il quarto piủ lungo di tulti.

\section{res: \\ Ptrima Sigzinte.}

Amitatori nei boschi o Silvestre.

\section{SPECIE LV.}

Turdus Viscivorus (Linneo).

Merle Draine.

Tordo Maggiore.

Turdu Colombinu o Turdu Suerinu C. M.

Turdu Colombimu o Turdu Suerimu C. S.

Parli superiori d'un bruno cenerino, le inferiori d'un bianco leggermente tinto di giallo rossiccio variato solto la gola ed il d'avanti del collo da macehie brune in forma di ferro di lancia, e nelle altre parti con delle macchie orali.

Si trotano in tulli i luoghi montagnosi ed in partieolare in quegli che abhondaun di ginepri, si vedono ordinariamente a copplie, vi nidificano, o partono primeipiando l'estate. 


\section{SPECIE LVI.}

Turdus Pilaris (Linneo).

Merle Litorne.

Tordello Fazzino.

Turdu C. M.

Turdu C. S.

Testa, nuca, e parte inferiore del dorso, cenerina, alto del dorso e copertnra delle ali castagni, gola e petto d'un rosso chiaro con delle macchie lanciolate nere, ventre d'un bianco puro, coda nera.

Passano annualmente in grandissimo numero sul principiar dell'autunno e partono in fin di primavera.

\section{SPECIE LVII.}

Turdus Musius (Linneo).

Merle Grive.

Tordo Botacico, o Tordo Gentile.

Turdu C. M.

Ismurtidu C. S.

Tutte le parti superiori d'un bianco tendente al color d'ulivo, gola bianca, parti laterali del collo e petto d'un giallo rossiccio con delle macchie triangolari brune: ventre e fianchi d'un bianco puro con delle macchie ovali brune.

Di passaggio annuale col precedente.

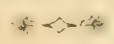




\section{SPECIE LVIII.}

Turdus Iliacus (Linneo).

Merle Mauvis.

Tordo Lassello.

Turdu C. M.

Turdu C. S

Tutte le parti superiori d'un bianco d'uliva : coperture inferiori delle ali, ed i fianchi d'un rosso ardente: parti laterali del collo e del ventre, ed il petto seminate di numerosissime macchie longitudinali nericcie; ventre d'un bianco puro.

Passa colle precedenti ma in iscarsissimo numero.

\section{SPEGIE LIX.}

Turdus Merula (Linneo).

Merle Noir.

Merlo.

Meurra C. M.

Merula C. S.

Tulte le piume d'un nero profondo, becen, interiore della bocca e contorno degli occhi gialli.

Comune, slazionario in tutte le parti dell'Isola, nidilica nei respugli. 


\section{Sicolion Sizione.}

\section{Amitatori delle mupi o Sassaicoli. SPECIE LX.}

Turdus Saxatilis (Latham).

Merle de Roche.

Codirosso Maggiore o Tordo Marino.

Solitariu Coa arrubia C. M.

Solitariu C. S.

Tutta la testa e l'alto del collo d'un azzurro cenerino o di piombo ; parti superiori d'un bruno nericcio, in mezzo al dorso un largo spazio bianco; ali e le due penne centrali della coda brune; le altre della coda, e le parti inferiori d'un rosso ardente.

Compariscono in autunno, abitano le rupi, e sopra le vecchic torri fanno d'ordinario il loro nido.

\section{SPECIE LXI.}

Turdus Cyames.

Merle Bleu.

Passera Solitaria.

Solitariu C. M.

Solitariu C. S.

Le parti superiori d'un bell'azurro carico, le inferiori similmente d'azzurro pii carico asperse di strellissime marchie in forma di luna crescente di color nero disposte verso la punta delle piume che sono terminate da altre macchie dell'istessil forma bianchicerie; ali c codla d'un nero profondo. 
Alle prime pioggie d'autunno si sente il bellissimo suo canto sopra il telto delle nostre case, nidifica fra noi, ebbi varie volte dei novelli, fa il suo nido nelle fessure delle roccie le più elevate e sopira gli scogli vicini al mare.

II genere Merlo, Turdus. Dappertutto ritrovasi.

\section{GENIRI XVII:}

\section{Cinclo. Cinclus (Becstein).}

Carattere generico. Becco mediocre, dritto, schiacciato, tagliente e rotondato verso l'estremità, mandibola superiore elevata colla punta curvata sull'inferiore: narici collocate alla base del becco, ed ai lati in una fessura longitudinale, coperte da una membrana, testa piccola, stretta alla sommita, colla fronte allongata e che viene a terminare alle narici, quattro dita, tre d'avanti e l'interno piu grande dei laterali che sono eguali, e saldato all'esterno verso la base, uno situato di dietro, libero, tarso piủ lungo del dito intermedio: primo remigio cortissimo, il terzo, e quarto più lunghi di tulti.

Questi uccelli vivono solitarj, e si ritirano nelle montagne, compariscono in pianura solamente nella stagione degli amori.

\section{SPECIE LXII.}

Cinclus Aquaticus (Bechstein).

Cincle Plongeur.

Merlo d'acqua.

Menra de Arriu o Sturru de acqua C. M.

Sturru de riu C. S.

Parli superiori d'un brumo opaco finto di renerino: gola. parte anteriore del collo e pefto d'un bianco puro ; ventre rosso. 
Stazionario nidifica fra noi, vive sulle sponde dei nostri fiumi, we trov a il suo alimento, che consiste in piccoli insetti acquatici.

II genere Cinclo Cinclus vive in Europa, Asia, e prolabilmente anche in America boreale.

\section{GENERE XIX}

\section{Beccir gentili Sylvia (Laham).}

Carattere generico. Becco dritto, delicato, in forma di lesina. a base piu elevata che larga: la punta della mandibola superiore sovente incavata, l'inferiore dritta. Narici poste ai lati della sta base in forma ovali, fermate a meti da una membrana. Piedi a tarso piu lungo di quello di mezzo, tre dita d'avanti, ed unn dietro; l'esteriore saldato alla base del dito di mezzo : unghia del dito di dietro di mezzana lunghezza ed arcuata. Ali, primo remigio molto corto o quasi nullo, qualche volta nullo, il secondo un poco piú corto del terzo o della medesima lunghezza : le grandi coperture alari molto piu corte dei remigj.

In questo genere si comprendono le specie più piccole degli uccelli, ed è composto di quegli che rallegrano i boschi col loro canto, che soventi è molto melodioso. Si nutrono aflatto d'insetti che cercano sui cespuglj sallando di ramo in ramo, o nei ruseelli; non hanno peró l'abitudine di prenderli al rolo eome fanno altri nccelli. 


\section{Mrima Sieziour.}

\section{Paludicole o Aritatori del rivi.}

\section{SPECIE LXIII.}

Sylvia Aquatica (Latham).

Bee fin Aquatique.

Pagliarola.

Una striscia bianca gialliccia passa sopra gli occhi, allara simile piu larga parte dalla base del becco, e va nel centro del cranio : nuca, parti laterali del collo, scapolari ed alto del dorso d'un grigio leggermente tinto di rossiccio, gli ultimi con delle macchie longitudinali nericcie.

Di passaggio annuale in primavera. Abita nei luoghi paludosi ( nei fiumi, ne feci varie volte caccia nei fossi d'acqua, ove cresce il Nasturzio (Nasturlium Aquaticum ).

\section{SPECIE LXIV.}

Sylvia Cetti (Della Marmora).

La Fauvette Cetti.

Rossignolo di Palude.

Passarilanti C. M.

Passarilanti C. S.

Le parti superiori della testa, del corpo, e delle ali di color bruno carico leggiermente tinto di rosso: parti laterali del collo, lianchi, coscie, e basso-ventre d'un bruno rosso meno carico di quello del dorso ; gola, parte inferiore del collo e centro del ventre d'un bianco puro.

Nidilica fra uni od comune in riva ai fumi in lutle le parti dell'isola. 


\section{SPECIE LXV.}

Sylvia Cisticola (Temminck).

La Fauvette Cisticola.

Becca Mosche.

Topi de Mala C. M.

Sommita della testa, nuca, dorso e coperture delle ali color di foglia morta che contorna tutte le piume di color bruno nericcio, cio che produce una moltitudine di larghe macchie disposte longitudinalmente : parte inferiore del dorso e groppone color di foglia morta: le parti inferiori d'un bianco rossiccio pii opaco sui fianchi.

Stazionario fra noi, e vi nidifica, abita nei giunchi she crescono in vicinanza del mare in luoghi umidi.

\section{ingit \\ Seruntio Sizinue.}

Silyestri, o Abitatori dei noscili.

\section{SPECIE LXVI.}

Sylvia Luscinia (Latham).

Bec fin Rossignol.

Rossignuolo.

Rossignolu C. M.

Rossignolu C. S.

Tutte le parti superiori d'un bruno tinto di rosso di ruggeine : gola e ventre bianchiccio, petto, o fianchi cenerini.

Tante volte arriva fra noi ai primi di marzo. Nidifiea noi luoghi ove crescono le canne. 


\section{SPECIE LXVII.}

Sylvia Philomela (Bechstein).

Bec fin Philomele.

Russignuolo forestiero.

Filomena C. M.

Filomena C. S.

P'arti superiori d'un grigio bruno appannato, il petto di color grigio tinto di grigio più oscuro, coda di color rosso men vivo vella precedente specie: gola bianca contornata di grigio carico. Comparisce fra noi in primavera. Nidifica nei luoghi umidi.

\section{SPECIE LXVIII.}

Sylvia Atricapilla (Latham).

Bec fin a tête noire.

Capinera.

Conca de Moru C. $\mathbf{M}$.

Conca de Moro C. S.

Filomena al maschio, Muschita alla femmina C. S.

Fronte, sommita della testa, e nuca d'un nero profondo, collo e petto d'un grigio cenerino, ventre e gola d'un cenerino bianchiccio: le altre parti superiori del corpo, le ali e la coda d'un cenerino leggiermente tinto d'olivastro.

Comparisce come la precedente, ma pii comume. 


\section{- it -}

\section{SPECIE LXIX.}

Sylvia Melanocephola (Latham).

Bec fin Mélanocephale.

Occhiocotto.

Conca de Moru C. M.

Conca de Moru C. S.

Fronte, sommiti della testa, nuca, guancie, ed orifizio delle: orecchie d'un nero profondo; gola, parte anteriore del collo, e centro del ventre, bianco; dorso, fianchi, basso-ventre e coperture delle ali d'un grigio carico: ali e coda nericcie.

Comune, stazionario, fa il suo nido negli alcini. Atriplex Alcinus.

\section{SPECIE LXX.}

Sylvia Sarda (Della Marmora).

Bec fin Sarde.

Occhiocotto Sardo.

Topi de Mata C. M.

Ogu de Oe C.S.

Sommita della testa, guancie, parte anteriore del collo, manto, dorso, e groppone d'un cenerino nericcio, più profondo sulla fronte : nuca, parti laterali del collo, petto e fianchi d'una tinta più chiara che prende il rossiccio o color di vino alle coscie : centro del ventre d'un bianco leggiermente tinto di color di vino.

Stazionario, preferisce vivere nei lugghi incolti e deserti, particolarmente nei montuosi. 


\section{- is -}

\section{SPECIE LXXI.}

Sylvia Cinerea (Latham).

Bec fin grisette.

Sterpazzola.

Stampacresuras C. M.

Sommita della testa cenerina: le altre parti del corpo di un wrigio fortemente tinto di rosso: ali nericcic e le coperture bordate d'un rosso vivissimo: gola, e centro del ventre d'un bianco puro, petto leggiermente tinto di roseo, lianchi e basso-ventre d'un grigio rossiccio.

Comune, e vi nidifica.

\section{SPEGIE LXXII.}

Sylvia Curruca (Latham).

Bec fin Babillard.

Bigiarella.

Topi de Mata C. M.

La parte superiore della testa d'un cenerino puro: nuca, manto, e groppone d'un cenerino bruno: petto, fianchi, e basso ventre d'un bianco leggiermente tinto di rossiccio : le altre parti inferiori d'un bianco puro.

Meno comune della precedente arriva in aprile. 


\section{SPECIE LXXIII.}

Sylvia Conspicillata (Della Marmora).

Bec fin à Lunettes.

Sterpazzola di Sardegna.

Topi de Mata C. M.

Sommita della testa, e guancie d'un cenerino puro, manto, e dorso d'un roseo di vino: ali nericcie colle coperture bordate d'un rosso vivo, gola d'un bianco puro, tutte le altre parti inferiori d'una tinta color di vino chiaro verso il centro del ventre, e rossiccio verso i fianchi.

Stazionario pure fra noi, abita in tutti i luoghi di boscaglie.

\section{SPECIE LXXIV.}

Sylvia Provincialis.

Bec fin Pitchou.

Magnanina.

Topi de Mata C. $\mathbf{M}$.

Tutte le parti superiori d'un bel grigio carico ad eccezione della coda che ha un bruno rossiccio: gola, petto e fianchi d'un rossiccio di porpora, o feccia di vino: centro del rentre bianco.

Comune nei luoghi montagnosi. 


\section{SPEGIE LXXY.}

Sylvia Passerina (Latham).

Bec fin Passerinette.

Sterpazzolina.

Topi de Mata C. 1 .

Sommita della testa, guancie, nuca, dorso e parte anteriore del collo d'un color di piombo; parti superiori del corpo d'un cenerino olivastro : gola, petto, e fianchi d'un rosso di mattone leggiermente violetto ; ventre e basso-ventre d'un bianco puro, o meno puro.

Anche rquesta specie stazionaria fra noi, ma si confonde colla femmina della Conspicillata, a cui assomiglia moltissimo.

\section{SPECIE LXXVI.}

Sylvia Rubecula (Latham).

Bec fin Rouge Gorge.

Pettirosso.

Barbarrubia Grisu C. M.

Fra Gavinu C. S.

Sommita della testa, e parti superiori d'un grigio bruno deincnte tinto d'olivastro: fronte, petto, e parte anteriore del

o d'un rosso ardente contornato questo dai lati d'un grigio rino: fianchi d'un cenerino olivastro: ventre d'un bianco

-azionario pure fra noi, e vi nidifica : passa l'estate in monisna, e all'inverno scende nelle pianure. 


\section{SPECIE LXXVII.}

Sylvia Tithiis ( Scopoli.

Bec fin rouge queue.

Codirosso Moretto.

Coa de fogu. C. M.

Fra Gavinu C. S.

Parti superiori d'un cenerino azzurrognolo: spazio tra it becco e l'occhio, guancie, gola e petto d'un nero profondo : il nero si sbiada in cenerino azzurrognolo sul ventre e fianchi : basso ventre bianchiccio, coperture inferiori della coda, groppone, e penne codali d'un rosso ardente.

Comune fra noi, preferisce vivere nelle roccaglie.

\section{SPECIE LXXVIII.}

Sylvia Phoenicurus (Latham).

Bec fin de Murailles.

Codirosso dei Monti.

Coa de ferru C. M.

Fra Gavinu C. S.

Fronte e sopracciglia d'un bianco puro: gola, ed alio de! collo d'un nero profondo: testa ed alto del dorso d'un cenerino azzurrognolo: petto, fianchi, groppone e penne laterali della coda d'un rosso brillante: basso-ventre bianchiccio : copertura inferiore della coda d'un rosso chiaro.

Comune nell'inverno abita come la precedente.

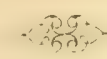




\section{Cerza Sezinne.}

\section{Muscivoni.}

Il loro nutrimento consiste principalmente in mosche, whe prendono al volo o su le foglie.

\section{SPECIE LXXIX.}

Sylvia Hippolais (Latham).

Bec fin a poitrine jaune.

Beccafico Canapino.

Topi de Mata C. M.

Parti superiori d'un cenerino leggiermente tinto di verde, uno strettissimo cerchio giallo attorno gli occhi: dalla gola alla copertura inferiore delle ali domina un giallo pallido: grandi coperture delle ali e della coda brune bordate di grigio verdastro.

Al principiare dell'inverno compariscono fra noi questi uccelli, vi sono comunissimi, e frequentano i nostri giardini massime degli agrumi.

\section{SPEGIE LXXX.}

Sylvia Sibilatrix (Becsthein):

Bec fin Siffeur.

Lui verde.

Sommita della testa, e tutle le parli superiori di un bel rerde 
chiaro: parti laterali della testa, gola, parte anteriore del collo, inserzioni delle ali e della coda d'un giallo puro: il resto delle. parti inferiori d'un bianco puro.

Di raro si fa vedere fra noi quest'uccelletto in primavera.

Il genere Silvia, Sylvia trovasi dappertutto.

\section{GENERT XX}

\section{Reattino. Regolus (Raii).}

C'irattere generico. Becco molto sottile, delicato, corto, e molto dritto, la mandibola superiore appena intaccata verso l'estremita: narici ovali coperte e nascoste da piccole piume dirette d'aranti. Piedi molto delicati: dito di mezzo riunito alla base dell'esterno, ed ì totalmente separato dall'interno ; il dito posteriore il piu forte di tulti. Ali di mediocre lunghezza, il primo remigio molto corto, il secondo e l'ottavo eguali, il quarto ed il quinto sono i piu lunghi.

\section{SPECIE LXXX!.}

Regulus Cristatus (Latham).

Roitelet ordinaire.

Testa d'oro.

Topi de Mata C. M.

Parti superiori del corpo d'un colore olivastro tinto debolmente di gialliccio : sulle ali due liste transversali bianchiccie : piume della sommità della testa lunghe, ed un poco affilate e t'un bel colore giallo vivo legriermente dorato: tulta la regione 
degli vechi, le parti laterali del collo, e le parti inferiori d'un cenerino tinto debolmente di rosso olivastro.

Pare che arrivi in autunno: un individuo ne fu predato in questa stagione nel giardino del $\mathbf{R}^{0}$ Convito dall'alunno cavaliere D. Giuseppe Falqui Pes. Lo vidi sempre nell'inverno, pii comune al Capo di Sassari.

\title{
SPECIE LXXXII.
}

\author{
Regulus Ignicapillus (Bustein). \\ Roitelet triple bandeau. \\ Fiorancino. \\ Topi de Mata C. M.
}

Parti superiori d'un verde olivastio che si unisce sui lati del collo al gialliccio: sulla sommiti della testa, e sulla nuca delle piume lunghe ed affilate color di fuoco risplendentissime accompagnate da ciascun lato di piume d'un nero profondo, quali riunendosi alla fronte vi formano una fascia transversale: le piume della fronte hanno una tinta rossiccia. Se ne fa preda d'inverno. ma di raro. II genere Reallino Regulus. Europa.

\section{GINIRE XXI.}

\section{Sericiolo. Troglodytes (Cuvier).}

Carattere generico. Becco molto gracile, fino, senza alcuni intaccatura, puntato, leggiermente arcato: mandibole eguali : atrici ovali, coperte da una membrana. Piedi lunghi, soltili: 
dito di mezzo riunito alla base dell' esterno, e affalto separato dall' interno. Ali molto corte, concave, ed arrotondate: primo remigio di mediana lunghezza, il quarto ed il quinto sono i piu lunghi. Coda cortissima.

Questi abitano le boscaglie, e non si mostrano allo scoperto che ben di raro. Amano l'oscurità.

\title{
SPECIE LXXXIII.
}

\author{
Troglodytes Vulgaris (Latham). \\ Troglodyte Ordinaire. \\ Sericiolo. \\ Pilloni de beranu C. M. \\ Sericiolu, o Puzzone e beranu C. S.
}

Parti superiori d'un bruno appannato macehiato da strellissime striscie transversali, gola e petto d'un bianco azzurrognolo : iutte le parti posteriori d'un color bruno con macchie bianche, e striscie transversali nere.

Comune in tutta l'isola ne ho preso tanto in primavera che nell' inverno.

Il genere Sericiolo, Troglodyles. Europa.

\section{GENIBE XEIT \\ Sassicole, Saxicola (Bechstein).}

Carattere generico. Becco dritto, gracile: base un po' piu larga che alta a lisca sporgente che s'avanza sulla fronte, le punte delle due mandibole in forma di lesina, la superiore sensibilmente 'urvata : alla base del beceo dei peli ben mareati : narici laterali 
nella base del becco in forma ovale alla meta fermata da una membrana : piedi a tarso il più sovente molto lungo, tre dita d'avanti, e uno dietro, l'esterno saldato alla base di quello di mezzo : unghia del pollice piú corta di questo, ma molto arcata : ali, il primo remigio assai lungo, il secondo un poco più corto del terzo e quarto che sono i piu lunghi: le grandi coperture alari molto piu corte dei remigi.

La maggior parte delle specie di questo genere ama di vivere nei luoghi incolti scoperti di selve e boschi : sono diffidenti, difficili ad ammazzare, perchè si ficcano fra le pietre e fessure delle rocche; si nutrono d'insetti che alcune specie inseguono correndo velocemente, ed altre che cogliono slanciandosi dall'alto ove volteggiano in traccia della preda.

\section{SPECIE LXXXI.}

Saxicola Cachinnans (Temminck).

Traquet Ricur.

Culbianco Abbrunato.

Culu biancu C. M.

Culu biancu C. S.

Tutte le parti del corpo d'un nero profondo: ali d'un nero brunito, groppone e copertura delia coda d'un bianco puro: Ie sole due penne codali di mezzo nere all'origine, tutte le altre hanno una striscia nera verso l'estremila che terminano con un punto bianco.

Abita nei luoghi di roccaglie, ne feci preda varie volte nelle rocelie di Tuvixieddu al di sopra di Sant'Arendrare. 


\section{SPECIE LXXXV.}

Saxicola OEnanthe (Bechstein).

Traquet Moteux.

Monachella.

Culu biancu C. M.

Culu biancu C. S.

Parli superiori del corpo d'un grigio cenerino. fronte e gola bianche : ali nere, due terzi della lunghezza della coda bianchi, l'altro verso la punta nero, ad eccezione delle due penne di mezzo che sono aflatto nere: la parte anteriore del collo leggiermente tinta di bianco rossiccio, ed il rimanente delle parti inferiori bianche.

Comparisce fra noi negli inverni piu rigidi, ma non in gran numero.

\section{SPECIE LXXXVI.}

Saxicola Stapazzina (Temminck).

Traquet Stapazin.

Monachella di gola nera.

Culu biancu C. M.

Culu biancu C. S.

Spazio tra l'occhio ed il becco, regione degli occhi e delle orecchie, tutta la gola, gli scapolari, e le ali d'un nero profondo: sominiti della testa, groppone, e le parti inferiori d'un bianco puro, nuca, ed alto del dorso d'un bianco rossiccio : coda bianca per la massima sua lunghezza solanente nera verso la punta, ad eccezione delle due penne di mezzo che sono nere, e le esteriori quasi del tutto. 
Comune fra noi e stazionario; nell' inverno si vede in pianura nei campi particolarmente posti in vicinanza delle saline: all'estate nei luoghi montagnosi.

\title{
SPECIE LXXXVII.
}

Saxicola Aurita (Temminck).

Traquet Oreillard.

Monachella,

Culu biancu C. M.

Culu biancu C. S.

Regione degli occhi e delle orecchie, e le ali d'un nero profouto; gola, parte anteriore del collo, tutte le parti inferiori, la testa, ed il groppone d'un bianco puro: nuca, ed alto del dorso leggiermente tinto di rossiccio: coda come la precedente.

Di passaggio in primavera, ma in scarso numero.

\section{SPECIE LXXXVIII.}

\author{
Saxicola Rubetra (Bechstein). \\ Traquel Tarier. \\ Stiaccino. \\ Sartiarellu o Concamom C. II.
}

Şommiti della testa, parti laterali del collo, e superiori del 'orpo di un bruno nericcio : le piume bordate di giallo rossiccio : gola e tratti longitudinali di ciascun lato del collo d'un bianco juro: parte anteriore del collo, e petto d'un bel rosso chiaro : sua gran macehia bianca sulle ali c coda.

Stazionario fra noi vi nidifica, ama vivere nei luoghi vicini al mare, ha l'abitudine di posarsi all'estremili delle piante, e salta di sasso in sasso. 


\section{SPECIE LXXXIX.}

Saxicola Rubicola (Bechstein).

Traquet Rubicole.

Saltinpalo.

Sartiarellu, o Conca de Moru C. M.

Tutta la testa, gola, coda, dorso, piccole coperture delle ali d'un nero profondo, le piume bordate leggiermente di rosso bruno: parti laterali del collo, alto delle ali e groppone d'un bianco puro, ali brune bordate di bruno piu chiaro: petto d'un rosso carico: le altre parli inferiori d'un rosso chiarissimo.

Come la precedente, però ama piủ i luoghi montagnosi, ore ne feci varie volte caccia.

Il genere Sassicola, Saricola. Antico continente.

\section{GENERT XXIT.}

\section{Accentore. Accentor (Bechslein :}

Carattere generico. Becco dritto, appuntato colla mandibola superiore incavata verso l'estremita, e che pur è come l'inferiore compressa su i margini : narici nude poste alla base del beceo, Iraforate in un'ampia membrana: piedi robusti, tre dita d'avanti e uno dietro, l'esterno dei quali saldato presso alla base di quello di mezzo, l'unghia del posteriore è più lunga, piu arcuata. La prima penna remigia è quasi nulla, la seconda quasi eguale alla terza che è la più lunga.

Le specie che cadono solto questo genere non si nutriscono di soli insetti, ma qualche volla, e massime nell'inverno di semenze e grani. 


\section{SPECIE XC.}

Accentor Alpinus (Bechstein). Accenteur Pégot ou des Alpes. Sordone.

Testa, petto, collo, e dorso cenerino marcato quest' ultimo sull'alto di grandi macchie brune : gola bianca a scaglie brune: ventre e fianchi d'un rossiccio mescolato di bianco e rossiccio: ali e coda d'un bruno nericcio.

Accidentale è fra noi il suo passaggio, un solo individuo ne fu predato in inverno, esiste nel nostro Musen.

\section{SPECIE XCI.}

Accentor Modularis (Cuvier).

Accenteur Mouchet.

Passera Scopagola.

Sommita della testa cenerina con delle macchie brune: part haterali del collo, gola, e ventre d'un cenerino azzurrognolo: grandi macchie d'un bruno rosso nel centro delle piume e coperture delle ali : fianchi e groppone d'un grigio rossiccio, ventre bianco.

Di passaggio annuale in primavera, non lo vidi mai d'inverno, abita nei cespugli e negli olmi, ore piu volte lo cacciai.

Il gemere Acrentore, Arentor. Antico Continente. 


\section{GENTE XIIV:}

\section{Cutiettole. Motacilda (Latham).}

Carattere generico. Becco dritto, gracile in forma di lesina, cilindrico, e angoloso fra le narici che sono situate alla base, e lateralmente, sono oroidi coperte per metí d'una membrana nuda, tarso di lunghezza doppia del dito di mezzo, tre dita all'innanzi l'esterno unito per la base a quello di mezzo, un dito di dietro la cui unghia i assai più grande delle altre, coda lunga, eguale, orizzontale: primo remigio nullo, secondo piu lungo, scapolari abbastanza lunghi da coprire l'estremita dell'ala ripiegata.

Le Motacille abitano i luoghi scoperti, giammai nelle forest: o giuncaje.

\section{SPECIE XCII.}

Motacilla Alba (Linneo).

Bergeronette Grise.

Cutrettola biancola, o Ballerina.

Coetla bianca C. M.

Culisaida bianca $\mathrm{C}$. S.

Fronte, guancia, parti laterali del collo e parti inferiori d'un bianco puro: nuca, gola, petto, peune di mezzo della coda, e copertura superiore delle ali d'un nero profondo, dorso e fianchi cenerini.

Di passaggio annuale, arrivano alle prime pioggie d'autunno, e partono al principiare dell'estate. Abitano nei campi e nei luoghi umidi in vicinanza del mare; s'introducono nei cortili e nei giardini; di raro si posano sugli alberi, ma uon vi si fermano piu di qualche minuto. 


\section{SPECHE XCIII.}

Motacilla Boarula (Linneo).

Bergeronetle Iaune.

Codizinzola.

Coela groga C. $\mathrm{H}$.

Culisaida groga C. S.

Parti superiori cenerine : groppone d'un giallo olivastro; sopra gli occhi, e sulle parti laterali del collo una fascia bianca: gola d'un nero profondo: le parti inferiori d'un giallo chiaro.

All'incominciare d'autunno si fa sentire in citti sopra i telti delle nostre case; parte dopo avervi nidificato.

Il genere Cultrettola, Motacilla. Trovasi solamente nell'antico Continente.

\section{GENER $\mathbf{X X V}$.}

\section{Spioncelle. Antuus (Bechstein).}

Ciarattere generico. Becco dritto, gracile, cilindrico, verso la punta in forma di lesina, a bordi piegati in dentro verso il mezzo ; base della mandibola superiore a lisca, punta leggiermente intaccata: narici laterali situate alla base, fermate a meti da una membrana vuota.

Piedi : tre dita d'avanti, ed uno dietro, l'esteriore saldato alla base del dito di mezzo, ed un dito dietro, unghie di dietro piu o meno curvate, il piu sovente eccede la lunghezza del dito posteriore. Ali : il primo remigio nullo, il secondo un po' piu corto del terzo e quarto che sono i piủ lınghi, le grandi coperture arrivano all'estremità dei remigj.

Le Spioneelle hanno le slesse abitudini delle Motacille. 


\title{
SPECIE XCIV.
}

\author{
Anthus Aquaticus (Bechstein). \\ Pipit Spioncelle. \\ Spioncella. \\ Pispanti C. M. \\ Pispante C. S.
}

Parti superiori d'un grigio bruno, sopra gli occhi una striscia bianca: tulte le parti inferiori bianche, ma variate ai lati del collo, al petto ed ai fianchi, con macchie Iongitudinali poco distinte d'un bruno cenerino chiaro.

Abbondante ne è il loro passaggio in autunno, e partono principiando l'estate. Abitano nei campi e nei luoghi paludosi in compagnia tante volte delle Cuttrettole.

\section{SPECIE XCV.}

\author{
Anthus Rufescens (Temminck). \\ Pipit Rousseline. \\ Ciarletto. \\ Fanfarroni C. M. \\ Fanfarrone C. S.
}

Parti snperiori del corpo d' un grigio isabella : in mezzo il ciascuna piuma una leggiera tinta bruna : sopra degli occhi una larga fascia bianca, e bianca la gola: tutte le altre parti inferiori d'un bianco isabella.

Arrivano fra noi in autumno, e partono terminando la pri-mavera, fanno il nido nei luoghi sabbiosi in terra come le Calandre. 


\title{
SPECIE XGVI.
}

\author{
Authus Pratensis (Bechstein). \\ Pipit farlouse. \\ Pispola. \\ Pispanti C. M. \\ Pispante C. S.
}

Parti superiori d'un cenerino olivastro con delle macchie nericcie nel centro di ciascuna piuma, piu grandi nelle piume del dorso : parti inferiori d'un bianco leggiermente tinto di gialliccio, ma variato sulle parti laterali del collo, del petto, dell'alto del rentre, e di tutta la lunghezza dei fianchi per mezzo di grandi macchie nere lunghissime e larghe: l'unghia del pollice piu lunga del dito, e debolmente arcuata.

Comunissimo fra noi, vi nidifica, e parte principiando l'estate: abita di preferenza nei luoghi umidi, e nei campi, ef è conosciutissimo da tutti.

\section{SPECIE XCVII.}

Anthus Rufogularis (Brisson).

Pipit à gorge rousse.

Pispola gola rossa.

Pispanti C. M.

Pispante C. S.

Sommita della testa, e nuca d'un bruno chiaro macchiato di striseie nere vicinissime: il manto, tutto il dorso e le ali a lunghe e larghe macchie nere contornate d'un bruno senerino: 
pelto, parte superiore del ventre, e fianchi coperti di grandi cordoni, e di piccole macchie nere disposte sopra fondo bianco, , isabella chiaro.

Di raro si fa preda di quest'uccello, forse perchè si confonde: colla specie seguente.

\section{SPECIE XCVIII.}

Anthus Arboreus (Bechstein).

Pipit de Buissons.

Tordino.

Pispanti C. M.

Pispante C. S.

Parti superiori d'un cenerino lavato d'olivastro con del bruno nericcio disposto longitudinalmente nel centro delle piume: il bianco gialliccio dell'estremila delle piccole, e medie coperture delle ali vi forma una doppia fascia transversale: gola d'un bianco puro: il rimanente delle parti laterali del collo e parte anteriore del medesimo, petto e fianchi d'un bel rosso gialliccio : sul petto delle grandi macchie nere in forma di pico, e sui fianchi dei tratti longitudinali strettissimi : il centro del ventre d'un bianco puro.

Comune fra noi, stazionario, frequenta i luoghi montuosi: nidifica nei cespugli, e negli alberi.

Il genere Spioncelle, Anthus trovasi dappertutto.

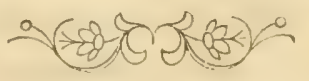




\section{-66 - \\ ORDINE IV.}

$-20.5$

\section{GRANIVORI-GRANIVORES.}

Becco corto, grosso, forte, piủ o meno conico, la cui lisca ordinariamente appiattita sporge sulla fronte: mandibola superiore d'ordinario senza incavature, tre dita d'innanzi e divise, uno di dietro: ali mediocri.

\section{GENERE XXVI.}

\section{Allodola. Allauda (Limeo).}

Carattere generico. Becco cilindrico, conico piu o meno allungato, piu o meno arcuato, e dritto, mandibola superiore vuota, leggiermente curva, guernita alla sua base di piccole piume dirette d'aranti: narici alla base del becco, rotondate, chiuse a meta da una membrana vuota: piedi, tre dita d'avanti, e uno dietro : il di mezzo saldato alla base dell' esterno, totalmente separato dall' interno. Unghie posteriori dritte, o leggiermente arcuate, sovente piu lunga delle dita. Ali; la prima penna remigia nulla o quasi nulla, la seconda un poco più corta della terza che è la piu lunga. Piume della nuca molto affilate, suscettibili ad erigersi in ciuffetto. II loro nutrimento consiste principalmente in granaglie o semenze, gli inselti serrono a loro d’alimento nel tempo destinato ad allevare i loro pulcini. Dopo 
i colombi ed i gallinater, sono quelli che si uniscono piit alJ'uomo, e somo i piu suscettibili essere addomesticali.

\title{
Hrima Sezione.
}

Questi hanno il beceo assai lungo, o piu lungo della testa leggiermente arcuato.

Questa prima sezione manca fra noi.

\section{Seranta Sizzinte.}

Becco un poro delicato, quasi dritto in forma di cono allungato.

\section{SPECIE XCIX.}

\author{
Allauda Arvensis (Linneo). \\ Alouette de Champs. \\ Allodola Campestre. \\ Calandria C. M. \\ Chilandra C. S.
}

Parti superiori d'un grigio rossiccio con delle macchie nerir(iv) nell'autro di ciascuna piuma, piit grosse nell'alto del dorso. c sulla testa. Sopra degli occhi una fascia bianchiccia : guancie dun bruno grigio: gola bianca; collo, pello e fianchi tinti di rossiccio : nel centro di ciascuna piuma una macchia bruna lanreolata. C'entro del ventre d'un bianco leggiermente tinto di rossiccio.

Esiste fra noi in grandissimo numern. Abita i campi, e particolarmente quelti del frumento. 


\title{
SPECIE C.
}

\author{
Allauda Arborea (Linneo). \\ Alouette lulu. \\ Lodolicchio. \\ Calandria C. M. \\ Chilandrina C. S.
}

Piume della testa un poco allungate, coda corta perfeltamente quadra. Parli superiori d'un cenerino rossiccio con del bruno nericcio nel centro delle piume: una striscia bianchiccia passa sopra degli occhi, e contorna l'occipite; le guancie, che sono brune hanno una macchia triangolare bianchiccia : le parti inferiori d'un bianco leggiermente tinto di gialliccio, ma variato sulla parte anteriore del collo, e sul petto da macchie longitudinali : comuni come la precedente. Un individuo di questa specie da me cacciato all'imboccatura della $S c a f f a$, ha il becco lungo un pollice, e quattro linee, ed è molto arcuato ; esiste quest'individuo nel nostro Museo, a cui ho dato nome d'Allauda arborea Deformirostro.

\section{SPECIE CI.}

\author{
Allauda Cristata (Linneo). \\ Alouette Cochevis. \\ Lodola Capellata. \\ Calandria C. M. \\ Chilandra C. S.
}

Piccola cresta coronale a piume allungate, nere nel mezzo, e contornate di cenerino : parti superiori del corpo, e delle ali d'un 
cenerino grigio: contorno degli occhi, gola, ventre, e basso ventre d'un bianco leggiermente tinto di gialliccio; una stretta fascia siegue la direzione della gola, e delle macchie longitudinali brune cuoprono il petto.

In piccolo numero comparisce fra noi in primavera, e parte nell'inverno.

\title{
SPECIE CII.
}

\author{
Allauda Brachidaclyla (Temminck). \\ Alouette Calandrelle. \\ Calandrella. \\ Taccaterra C. M. \\ Accucaditla C. S.
}

Tutte le parti superiori d'un bel rosso isabella, piủ cenerino sulla nuca: gola, e fascia sopra degli occhi d'un biarco puro: petto e fianchi d'un rosso chiaro; ventre, e basso-ventre d'un bianco leggiermente tinto di rossiccio.

Stazionaria fra noi.

\section{Cerza Sezione.}

Becco grosso, robusto, piu alto, che largo.

\section{SPECIE CIII.}

\author{
Alauda Calandra (Linneo). \\ Alouette Calandre. \\ Calandra. \\ Calandrioni C. M. \\ Chilandra Reale C.S.
}

Parti superiori del corpo d'un renerino rossiccio, con del brumo 
in mezzo alle piume: queste macchie sono piu larghe nel centro del dorso: gola ventre e basso-ventre d'un bianco puro : una gran macchia nera a ciascuna parte laterale del collo; fianchi, e petto d'un bianco tinto di color d'ocra: sul petto delle macchie lanceolate brune.

Stazionaria, nidifiea fra noi, ed è comunissima in tutte le. stagioni.

II genere Lomola, Alauda trovasi dappertutto.

\section{GENERE XXVTI. \\ Cingaleggra. Parus (Limeo).}

Carattere generico. Becco assai forte, dritto, conico, compresso, corto, aguzzo, non incavato, guarnito di peletti alla base, e tagliente ai bordi delle mandibole: mandibola superiore talvolta un poco curva verso le narici siluate a ciascuna parte del beco, e la punta presso alla base rotonda, equasi intieramente occultata da piccole pennetle dirette innanzi: piedi assai robusti: quattro dita, tre d'aranti intieramente divise, uno di dietro coll'unghia più forte, e piu adunca che negli altri: il primo remigio di mediocre lunghezza, o quasi nullo, il secondo molto menu lungo del terzo che oltrepassa tutti gli altri, piü corto pure del quarto e del quinto : questi uccelli hanno vivacita, coragraio, efl anche una certa ferocia : ecco i tratti piu marcati delle Cingallegre. Si nutrono d'inselti, di frutla, e di semenze, soprallutlo delle oleaginose, che sono per esse un cibo favorito, non mastirano punto le sostanze vegetali come fanno la maggior parte degli uccelli granivori, ma le spezzano a colpo di bece, e ne inthiottono i frammenti, the usano l'attenzione di mondare dit ogni materia che unn potrehbe essere digerita. Talsolta diven- 
Lando ancora piu omnivore si gettano sulle carogne per distaccarne piccoli brani, finalmente visitano i nidi delle piccole specie degli uccelli, spiano i momenti d'assenza della covatrice, attacsano la prole senza difesa, e la distruggono spaccandone il cranio per succhiarne le cervella.

\section{बदि \\ Yrima Sizione.}

Abitatori dei boschi.

\section{SPECIE CIV.}

Parus Major (Linneo).

Mèsange Charbonnier.

Cingallegra maggiore.

Accuzzaferru C. M.

Conca de moro C. S.

Testa, gola, parte anteriore del collo, ed un tratto longitudinale nel centro del ventre d'un nero a riflessi : tempia d'un bianco puro; manto d'un verde olivastro : groppone e piccole coperture delle ali cenerine: parti laterali del ventre giaile, ali bordate di cenerino con una striscia transversale bianca.

Stazionario nidifica sopra gli alberi più alti.

\section{SPECIE CV.}

Parus Ater (Linneo).

Mésange petile. Carbonnier.

Cingallegra minore.

Accuzza ferru C. M.

Sommita della testa, nuca, gola, e d'avanti del collo d'un 
nero profondo: una larga fascia bianca sulle parti laterali del collo, ed un grande spazio cosi stesso bianco sulla nuca : parti superiori cenerine: due striscie transversali bianche sulle ali : ventre bianco ; fianchi e basso-ventre grigj.

Comparisce fra noi rarissimamente qualche volta nei piu freddi inverni.

\title{
SPECIE CVI.
}

\author{
Parus Coeruleus (Linneo). \\ Mesange bleue. \\ Cingallegra celeste. \\ Acuza ferru C. II.
}

Sommita della testa d'un azzurro chiaro collana del basso del collo, e striscia transversale delle tempia d'un azzurro piu chiaro: fronte, sopracciglia, corona occipitale, e tempia d'un bianco puro : alto del dorso d'un verde olivastro: una striscia transversale bianca sulle ali; gola e tratto longitudinale sul centro del ventre d'un nero azzurrognolo: petto, parti laterali del ventre, e basso ventre d'un bel giallo.

Meno comune della prima, vi è stazionaria, e vi nidifica.

La seconda e terza sezione di questo genere manca fra noi.

Il gener Cingallegra, Parus trovasi dappertulto.

\section{GENERE XXVIT. \\ Zigola. Emberizza (Limeo).}

. Carattere generico. Becco corto, forte, conico, compresso latcralmente, appuntito, tagliente, bordo delle mandibole rientrante. queste somo distanti l'una diall'altra alla loro base, la su- 
periore meno larga dell'inferiore è guernita internamente d'un piccolo tubercolo osseo : narici situate alla base, ritondate, coperte in parte dalle piume della fronte: piedi tre dita d'innanzi intieramente divise, uno dietro ; primo remigio un po' più corto del secondo, e del terzo che sono i piu lunghi. Questi uccelli sono assai ricercati in altri luoghi, ve ne ha qualche specie che ligura con distinzione sulle mense, e sono riserbate a quelle dei ricchi.

\section{żilrima Sezinte.}

\section{Zigoli (propriamente delli).}

\section{SPECIE CVII.}

Emberizza Melanocephala (Scopoli).

Bruant Crocole.

Zigolo.

Orgiali C. MI.

Cincirri C. S.

Tutta la sommita della testa, regione degli occhi e delle orecchie d'un nero profondo : parti laterali del collo, e generalmente tutte le parti inferiori d'un giallo di cedro: nuca, dorso, scapolari, e groppone d'un rosso chiaro: ali, e coda d'un bruno chiarissimo; tutte le piume, e le penne orlate di bianco.

Di passaggio accidentale in primavera: ne feci caccia d'un solo individuo in piume da giovine; esiste nel nostro Museo. 


\title{
SPECIE CVIII.
}

Emberiza Citrinella (Linneo).

Bruant Iaune.

Zigolo giallo.

Orgiali C. $\mathbf{M}$.

Cincirri C. S.

Testa, guancie, parte anteriore del collo, ventre, e copertura inferiore della coda d'un bel giallo: macchie rossiccie sul petto r fianchi; piume dell'alto del dorso nericcie al centro, e rossiccie ai bordi, quelle del groppone d'un castagno chiaro, grigio all'estremità.

Arriva fra noi in primavera, vi annida, e parte in ottobre. Trovasi piu comune in montagna, che in pianura.

\section{SPECIE CIX.}

\author{
Emberiza Miliaria (Linneo). \\ Bruant Broyer. \\ Ortolano Strilozzo. \\ Orgiali de denti. C. $\mathbf{M}$. \\ Orgiale, e Cincirri a dentes C. S.
}

Parti superiori d'un bruno cenerino a macchie longitudinali nere: gola bianca marcata lateralmente, ed al centro con delle piccole macchie nere: centro del ventre, e basso ventre bianco: ali, c coda d'un nericcio cenerino.

Fa stazione fra noi, ed è comunissimil. 


\title{
SPECIE CX.
}

Emberizza Schoeniculus (Linnco).

Bruant de Roseau.

Monachino di Palude.

Orgiali C. M.

Orgiale C.S.

Testa, occipite, guancie, gola e parte anteriore del collo d'un nero profondo: una piccola striscia bianca si prolunga nelle parti laterali del collo principiando a poca distanza dagli angoli del becco: nuca, parte inferiore del collo, parti laterali del petto, ventre, e basso-ventre d'un bianco puro: delle macchie longitudinali nere sui fianchi, ed una larga striscia longitudinale d'un nero profondo nel centro di ciascuna piuma.

Di passaggin nell' inverno, ma in scarso numero.

\section{SPECIE CXI.}

\author{
Emberiza Hortulana (Linneo) \\ Bruant Ortolan. \\ Ortolano.
}

Gola, e cerchio attorno degli occhi di color giallo: testa, e collo d' un grigio olivastro con delle piccole macchie brune: piume delle parti superiori d'un grigio rossiccio ai bordi, e nericcio al centro: piume del petto, ventre, e bassomrentre d'un rosso brunn terminate di cenerino: coda nericcia.

Di passaggio accidentale. 


\title{
SPECIE CXII.
}

\author{
Emberizza Cirlus (Linneo). \\ Bruant zizi ou de Haie. \\ Zigola comune. \\ Pispanti C. M. \\ Pispante, e $Z i-z i \mathrm{C}$. S.
}

Gola, ed alto del collo d'un bel nero: una fascia cosi stesso nera passa solto gli occhi principiando dagli angoli del becco: un'altra di color giallo brillante forma sopra gli occhi un largo sopracciglio, che si protende alla nuca : una terza dell'istesso colore passa sopra degli occhi : sul basso del collo una larga macchia gialla : petto d'un cenerino olivastro; parti laterali del medesimo, e del ventre d'un bel rosso castagno ; ventre e basso ventre d'un giallo chiaro: testa e nuca di colore olivastro con delle piccole macchie nere : piume del manto d'un rosso castagno ai bordi, e nere nel mezzo.

Di passaggio annuale in primavera, e vi annida.

\section{SPECIE CXIII.}

Emberizza Cia (Linneo).

Bruant fou ou de pré.

Zigola mucciato.

Pispanti C. M.

Pispante C. S.

Parte anteriore del collo, e pelto d'un cenerino azzurrognolo puro: una fascia nera attraversa gli occhi, contorna la regione Welle orecchie, si rimises all'angolo del becco: un ampio soprar- 
ciglio bianchiccio sopra degli occhi seguito da una macchia nera, che si prolunga sopra la nuca : alto della testa cenerino con delle piccole macchie nere: piume del dorso, edelle ali d'un rosso cenerino con delle macchie longitudinali nere: ventre, fianchi, e basso-ventre d'un rosso puro.

Raro si fa vedere fra noi quest'uccello, qualche volta in aprile. La seconda sezione ad unghia posteriore lunga, e quasi drilla manca fra noi.

Il genere Zigola, Emberizza trovasi dappertutto, fuorchì nell' America Meridionale.

\section{GENERE XXIX \\ Lossia. Loxia (Brisson).}

Carattere generico. Becco mediocre, forte, molto compresso, le due mandibole parimenti curve, adunche; l'estremila incrocicchiantisi, narici laterali, rotonde, poste verso la base, e nascoste da setole dirette imnanzi, tre dita d'avanti, divise, uno di dietro, ali mediocri; il primo remigio il piu lungo; coda forcuta.

Questi uccelli abitano in tutti i paesi nei quali nasce spontaneamente il pino, dal frutto del quale essi traggono il principale loro alimento ; sanno sbucciare con molta destrezza il cono legnoso, nè vi lasciano vestigio della mandorla favorita. Allorchie viene a mancare loro questo cibo getlansi sopra ogni sorla di semenze. 


\title{
SPECIE CXIV.
}

\author{
Loxia Curvirostra (Linneo). \\ Bec-Croisé Commun ou des pins. \\ Becco in croce dei pini. \\ Biccu trottu C. M. \\ Bicen tortu C. S.
}

Color principale delle piume, rosso, che varia piu, o meno da quello di mattone al vermiglio: fronte, guancia, e sopraceiglia grigie con delle macchie gialliccie e bianchiccie: dorso, piccole coperture delle ali, e scapolari verdastri : groppone giallo, parti inferiori d'un verde giallicio : basso-rentre grigio con delle macchie più opache.

Di passaggio accidentale qualche volta d'inverno.

Il genere Lossia, Loxia trovasi nelle regioni boreali dei due Continenti.

Nota. Il genere 30. Pyrmla manca fra noi.

\section{GENEREXXXI.}

Frixguelut, Fringula (Illiger).

Carallere generico. Becco corto, robusto, convesso, dritlo, e conico in tutti i sensi : mandibola superiore rigonfia, lievemente inclinata alla punta, spesso prolungata in angolo, tra le piume della fronte: narici situate presso la base del becco, dietro l'elevazione cornea della parte connessa quasi totalmente nascosta dalle penne frontali : quattro dita, tre d'avanti intieramente divise, l'intermedio piu lungo del terzo: ali corte, i due o tre primi remigii raffilati, il terzo, o il quarto piii lunghi di lulti : i 
fringuelli traggono il principale loro nutrimento dai grani, dei quali rifiulano il guscio, of invoglia cortecciale spesso durissima, con molta destrezza: nel tempo degli amori fanno anche uso di larve, e d'inselti, gran numero tra essi vivono in societi : rinnovano una ed anche due volte all'anno la deposizione delle uora : per la maggior parte i maschi subiscono una doppia muta, " prendono nella stagione degli amori una veste splendidissima, che talvolta non rassomiglia per niente alla lor penna d'inverno. La bellezza di quella spoglia, ed in parecchi la meloria del canto, li fa dagli amatori ricercare: sommettonsi facilmente alli cattivita, e pare anzi riconoscano la mano, che li nutrisce.

\section{- क्रदs: \\ Primn Sezinne.}

Laticon.

A becen grosso, curvo piu, o meno rigontio ai lati.

\section{SPECIE CXV.}

Fringilla Coccothraustes (Temminck).

Le gros bec vulgaire.

Frosone.

Pizzugruzzu C. M.

Bicchirussu C. S.

Testa, guancia e groppone d'un bruno rosso, ma pií chiarn sulla fronte : contorno del becco, spazio tra questo, l'occhio, e la gola d'un nero profondo: una larga collana cenerina sulli nuca : manto di bruno carico: sulle ali una macchia longitudinale bianca.

Di passaggio amnuale in seltembre, in alcuni anni numernsissimo, in altri scarso. Parle principiando l'estate. 


\section{SPECIE CXVI.}

\section{Fringilla Chloris (Temminck).}

Gros-bec verdier.

Verdone:

Verdarolu C. M.

Canariu Areste C. S.

Tutte le parti superiori, ed inferiori del corpo, scapolari, e piccole coperture delle ali d'un verde gialliccio : coperture mediocri, penne secondarie delle ali cenerine con delle macchie nere. Comunissima fra noi, e vi nidifica.

\section{SPECIE CXVII.}

Fringilla Petronia (Linneo).

Gros-bec Soulcie.

Passera Alpestre.

Crucculeu de Monti C. M.

Furfurinu de Monte C. S.

Tutto il fondo delle piume di un bruno cenerino mescolato di bianchiccio nelle parti inferiori sopra degli occhi: un sopracciglio di bianco rossiccio susseguito da una fascia bruna piu larga, che si protende fino alla nuca; le parti superiori variate di bruno opaco hanno tutte le piume terminate di bianchiccio : una macchia di giallo vivo alla parte anteriore del collo.

Di passaggio annuale in autunno, nidifica nelle nostre monlagne. 


\title{
SPECIE CXVIII.
}

\author{
Fingilla Hispaniolensis (Temminck). \\ Gros-bec Espagnol. \\ Passera Sarda. \\ Crucculeu C. MI. \\ Furfurrinu, Biddisò C. S.
}

Sommita della testa e nuca d'un castigno vivo, e molto catrico; dorso, e manto nero, ma lutte le piume bordate lateralmente d'un rosso gialliccio: gola, parte anteriore del collo, ed un cinturone streltissimo sul petto d'un nero profondo, ma distribuito in lunghissime macchie sui fianchi, di modo che il solo centro del ventre, e del basso-ventre sono d'un bruno puro.

Comune, stazionario nidifica nei buchi dei vechi muri, e nei tetti delle case rustiche, fa due covate all'anno in autunno, e in primavera.

\section{SPECIE CXIX.}

Fringilla Montana (Linneo).

Gros-bec Friquet.

Passera Mattugia.

Sommita della testa, e rosso di rame: spazio tra l'occhio ed il becco, fascia sopra gli occhi, piume dell'orifizio delle orecchie, gola, ed una porzione della parte anteriore del collo d'un nero profondo: tempia, ed una collana interrotta sulla nuca d'un bianco puro: ali e coda d'un bruno opaco: piume del dorso e 
degli scapolari neri nel mezzo, e bordati di castagno; due fascie bianche sulle ali; petto d'un cenerino puro, ventre, e basso-ventre bianchicci.

In inverno, ma rarissimamente si fa vedere fra noi.

\title{
SPECIE CXX.
}

\author{
Fringilla Serinus (Linneo). \\ Gros-bec serin ou Cini. \\ Raperino. \\ Canariu de Monti C. M. \\ Canariu Areste C. S.
}

Fronte, contorno degli occhi, guancie, e fascia sopra degli occhi che si protende alla nuca d'un giallo verdognolo : parti superiori color d'olivastro accordato con del cenerino, con delle macchie nericcie: di color gionchiglio il groppone e petto, e questo ondato di cenerino: sulle ali due fascie transtersali una d'un giallo verdognolo, l'altra d'un bruno gialliccio : ventre d'un bianco gialliccio.

Di passaggio annuale in primavera.

Sicomin Sezinne.

B REVICONI.

Becro in forma di cono piu, o meno corto, drillo, e cilindrico, sovente conico, dappertulto. 


\section{SPECIE CXXI.}

- Fringilla Coclebs (Linneo).

Gros-bec Pinson.

Fringuello

Fringuellu C. 11.

Fringuellu C. S.

Fronte nera, alto della testa, e nuca d' un azzurro cenerino puro: dorso e scapolari di color castagno dolcemente accordato coll'olivastro: groppone verde: tutte le parti inferiori di color di feccia di vino rossiccio, che schiarisce sul ventre, e diviene bianchiccio sul basso-ventre: ali, e coda neri: due fascie transversali bianche sulle ali.

Stazionario fra noi ne' Junghi di montagna, raramente si fa vedere in pianura.

\section{SPECIE CXXII.}

Fringilla Camnabina (Linneo).

Gros-bec Linotte.

Fanello.

Passarellu C. M.

Fronte e petto d'un rosso cremisi, gola, e parte anteriore del collo bianchicci, con delle macchie longitudinali brune: sommitá della testa, nuca, e lati del collo d'un cenerino puro: dorso, scapolari, e coperture delle ali di un bruno castagno : fianchi d'un bruno rossiccio : centro del ventre, e basso-ventre bianchi.

Stazionario, ed ex comune in tutta l'Isola. 


\section{Cerza Sizinue.}

\section{LARgiconi.}

Becco a cono dritto, lungo, e compresso: colla punta delle due mandibole aguzza.

\section{SPEGIE CXXIII.}

Fringilla Citrinella (Linneo).

Gros-bec Venturon.

Lucaro Corso.

Canariu de monti C. M.

Fronte, sommita della testa, contorno degli occhi, gola, parte anteriore del collo, petto, e centro del ventre d'un verde gialliccio : nuca, lati del collo, e fianchi cenerini: dorso, scapolari, coperture delle ali, ed una fascia transversale su queste d'un verde gialliccio oscuro: groppone d'un giallo verdognolo: ali e coda nere.

Di passaggio in primavera, e vi nidifica.

\section{SPECIE CXXIV.}

Fringilla Spinus (Linneo).

Gros-bec Tarin.

Lucarino.

Canariu de monti C. M.

Sommità della testa, e gola d'un nero profondo: nuca d'un 


\section{$-85-$}

nero variato di verdognolo: una larga fascia gialla dietro grli occhi : collo, petto e ventre gialli : le piume del dorso, e scapolari d'un verdastro cenerino con una macchia longitudinale nericcia : due striscie sulle ali, una nera, e l'altra verde gialliccia : fianchi, e basso-ventre bianchicei con delle macchie longitudinali nere.

Di passagrio annuale in primavera, ed in autunno.

\section{SPECIE CXXV.}

Fringilla Carduelis (Linneo).

Gros-bec Cardonneret.

Cardellino.

Cardanera C. M.

Cardellina C. S.

Contorno del becco, e nuca d'un nero profondo: fronte, gola cremisi : guancie, parte anteriore del collo, e parti inferiori di un hianco puro: dorso, scapolari, e parti laterali del petto d'un bruno carico: meta superiore delle penne delle ali d'un giallo puro, l'altra nera con delle bianche verso la punta.

Comunissima fra noi, e vi nidifica.

Il genere Fringuello, Fringilla trovasi dappertulto.

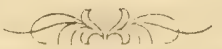




\section{$-86-$}

\section{ORDINE V.}

\section{0
-50}

\section{ZIGOIDATTILI. ZIGOI)ACTYLI.}

Due dita innanzi, e due dietro, becco piu, o meno curvo: dito esterno posteriore, talora reversibile.

\section{GENERE XXII.}

Cuculo. Cuculos (Linneo).

Carattere generico. Becco meliocre della lunghezza della testa, leggiermente arcuato, compresso: mandibole non incavate, narici poste alla base del becco, e presso i bordi della mandibola, circondata da una membrana nuda, e prominente: piedi piumati sotto il ginocchio assai corti : due dita di avanti saldate alla base, e due di dietro interamente divise, l'esterno delle quali reversibile: coda lunga ordinariamente raffilata : dieci rettrici: ali mediocri : il primo remigio di lunghezza media, il secondo alquanto più corto del terzo, che è il piủ lungo di tulti.

Le femmine dei Cuculi portano a deporre il frutlo dei loro amori in nidi estranei sorente ancora in quelli di piccolissime specie di silvie. Questi uccelli non si pascono che d’insetti, di larre, e vermi, il che li confina nei paesi caldi, né li porta a visilare i climi temperati se non nella slagione nella quale vi si mostrano gli inselli. 


\section{SPECIE CXXVI.}

Cuculus Canorus (Linneo).

Coucou Gris.

Cuculo.

Cucu C. M.

Cucu C. S.

Tutte le parti superiori, il collo, ed il petto d'un cenerino azzurrognolo, ma più oscuro sulle ali, ventre, coscie, basso ventre, e coperture inferiori della coda bianchiccie con delle striscie transversali d'un bruno nericcio: bordo membranoso del becco, e contorno degli occhi d'un giallo arancino.

Arrivano in primavera, e partono terminando l'estate.

Il genere Cuculo Cuculus trovasi in tutte le regioni temperate.

\section{GENERI XXII}

Picir. Picus (Linneo).

Caratteregenerico. Becco lungo, o mediocre, dritto, angolare, compresso, tagliente soprattutto verso la punta, che dall'uso si trova soventi smozzata; spicolo dritto; narici collocate alla base del becco, ovali, aperte, celate da peli diretli in avanti, piedi robusti, alti ad arranpicarsi, d'ordinario quattro dita, di raro tre, due d'innanzi, due o un solo di dietro, queste intieramente divise: le prime saldate alla loro base; coda composta di dodici rettrici, ed alle volte dieci soltanto, due laterali cortissimi a fusto, o verghetla forte, rigida, elastica, terminata da una punia, che serve a farne un punto di appoggio solido ; ali mediocri, il primo remigio cortissimo, il terzo, o il quarto più lunghi. 
Questi uecelli stanno di continuo in traccia d'una piccola preda, che mai basta a soddisfare completamente il loro appetito, si vedono tutto il giorno attorno il tronco degli alberi, percorrerne la superficie in tulti i versi colpendone col becco grosso, e robusto la corteccia soventi ribelle, onde scacciarne l'insetto seminato che riparato dalle vicende atmosferiche, attende l'epoca delle sue ultime metamorfosi. Le formiche prestano grandi risorse ai Pichi, quando mancano gli insetti sotto la corteccia degli alberi, ricorrono essi ai formicaj, si meltono in agguato all'ingresso d'un androne solterraneo, vi insinuano la lingua, ve la lasciano fin(hè un numero sufficiente di formiche ne copra le pareti, la ritirano, ingoiano gli insetti, e rinnovano la stessa operazione finche abbiano quasi spopolato tuta la repubblica.

\title{
SPECIE CXXVII.
}

\author{
Picus Viridis (Linneo). \\ Pic Vert. \\ Picchio verde. \\ Bicea linna C. M. \\ Attaccador $\approx a$ C. S.
}

Sommita della testa, nuca, e mustacchj d'un rosso brillante; faccia nera; parti superiori d'un bel verde; groppone tinto di gialliccio; parti inferiori d'un cenerino verdastro.

Piu comune verso la parte settentrionale dell'Isola. 


\title{
SPECIE CXXVIII.
}

\author{
Picus Major (Linneo). \\ Pic Epeiche. \\ Picchio rosso maggiore. \\ Bicca lina C. M. \\ Attaccador $\approx a$ C. S.
}

Fascia transversale bianchiccia sulla fronte: sommita della testa nera : uno spazio rosso sulla nuca; una larga fascia nera parte dall'angolo del becco contorna le tempia e si unisce da una parte sulla nuca, intantochè dall'altra si avanza allargandosi sino al petto; dorso, ed ali d'un nero profondo; tempia una macchia sulla parte laterale del collo, scapolari, e parti inferiori d'un bianco sporco : basso-ventre, e copertura della coda cremisi.

Comunissimo, e vi nidilica, chbi varie volte dei novelli.

\section{SPECIE CXXIX.}

Picus Minor (Linneo).

Pic Epcichetle.

Picchio Piccolo.

Bicca Linna C. M.

Allaccador:a C. S.

Tutta la fronte, regione degli occhi, lati del collo, parti inferiori d'un bianco appannato; delle striscie strette longitudinali sulpetto, e sui fianchi; sommita della testa rossa ; nuca, alto del dorso, e delle fascie nere, c bianche : una fascia nera va dall'angolo del becco sopra i laci del collo.

Trovasi in inverno: di raro peró se ne fa preda.

II genere Picchio, Piccus trovasi dappertulto. 


\section{GENERE XXIV.}

\section{Torgicollo Iune (Linneo).}

Carallere generico. Becco corto, dritto, conico, depresso, sotlile verso la punta, a lisca rotondata; mandibole non intaceate: narici alla base, aperte nel bordo concavo della lisca, nude, in parte fermate da una membrana; piedi, tre dita d'avanli saldate nella loro origine, due di dietro divise; ali mediocri, il primo remigio un po' più lungo del secondo, che è il piu lungo: questi ucelli non si arrampicano su gli alberi come fanno i Picchi, la poca solidita delle penne della coda non permette loro questo movimento di salire, fanno uso di formiche all'istessa maniera dei l'ichi introducendo la lingua nel loro nido.

\section{SPECIE CXXX.}

Iunx Torquilla (Linneo).

Torcol Ordinaire.

Torcicollo.

Fromigaju, e Papa fromiga C. $\mathrm{H}$.

Il londo delle piume delle parti superiori d'un cenerino rosso macchiato irregolarmente di bruno, e nero: una larga fascia bruna s'estende dalla nuca sino all'alto del dorso ; sulle barbe esteriori delle penne alari delle macchic quatrate rosse; gola, e parte anteriore del collo rossiccio con delle piccole striscie transrersali ; le altre parti inferiori bianchiccie seminate di macchie triangolari. Comune in primavera, e nidifiea fra noi.

II genere Torricollo, Iuns trovasi dappertulto. 


\section{$-91-$ \\ OR D I VE VI. \\ 1) 11}

\section{ANISOIDTTILI. ANISODACTILI.}

Becco piu , o meno curvo, spesso dritto, sempre lesinato, affilato, gracile, meno largo della fronte, tre dita d'innanzi, l'esterno saldato inferiormente al medio, uno posteriore, spesso lunghissimo: tulti forniti d'unghie lunghe e curve.

Nota. Il genere 35 e 36 Sitta, e Certhia manca fra noi.

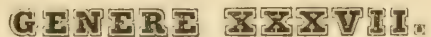

\section{Ticonroma. Tichodroma (Illiger.}

C'uruttere generico. Becco molto lungo leggiermente arcuato, gracile, cilindrico, a base angolare, colle punte depresse. Narici alla base nude, dirette orizzontalmente, fermate a meta da una membrana vuota. Piedi, tre dita d'avanti, l'esterno saldato alla base del dito di mezzo, uno di dietro fornito d'unghia molto lunga. Coda rotondata a verghette delicate. Ali larghe, primo remigio corto, secondo, e terzo gradatamente, quarto, quinto, e sesto i piủ lunghi.

Questi uccelli hanno l'abiludine di rampicarsi nei piani verlicali delle rocche, e dei vecthi edifizii, di raro su gli alberi. Il Ioro nutrimento si compone d'inselli, e larre. 


\section{SPECIE CXXXI.}

Tichodroma Phoenicuptera (Temminck).

Ticodrome Echélette.

Picchio In rajuolo.

Bicca Muru C. M.

Sommita della testa d' un cenerino carico, nuca, dorso, e scapolari d'un cenerino chiaro; gola, e parte anteriore del collo d'un nero profondo, parti inferiori d'un cenerino nericcio : copertura delle ali, e parti superiori delle barbe esterne delle penne d'un rosso vivo : estremita delle penne alari nere, con due grandi macchie bianche sulle barbe interne.

Piutlosto raro, ne feci preda sulle rupi di Sant' Elia, e dietro Buonaria, ma mai in perfetta livrea. Esistono due individui nel nostro Museo.

Il genere Ticodromo, Ticodroma, trovasi solamente in Europa.

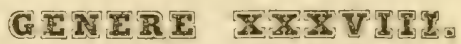

Burola. Upopa (Linneo).

Farattere generico. Becco molto lungo, leggiermente arcuato, sottile, triangolare, compresso : narici laterali poste alla base, in forma ovali, aperte, sormontate dalle piume della fronte. Piedi, tre dita avanti, l'esterno saldato alla prima articolazione del dimezzo, uno di dietro. Unghie corte, poco curve, quella del dito di dietro quasi dritta. Coda quadrata , composta di dieci penne. Ali mediocri, il primo remigio di mezzana lunghezza, il secondo, ed il terzo meno lunghi del quarto, e quinto, che sono i più lunghi. 
Le Bubole fanno in terra quel che fanno gli uccelli rampicanti sugli alberi, e su le muraglie, percorrono queste i terreni particolarmente gli umidi per dissotterrarne le larve, e gli inselti, che ivi si generano.

\section{SPECIE CXXXII.}

Upupa Epops (Linneo).

La Huppe.

Bubola.

Pubusa C. M.

Pubuza C. S.

Due ordini di lunghe piume rosse terminate in nero formano sulla testa un ciuffo arcuato: testa, collo, e petto d'un vinato rossiccio : alto del dorso grigio vinato : una larga fascia transversale sul dorso: le ali e la coda nere : basso-ventre bianco, con qualche macchia longitudinale sulle coscie.

Arrivano fra noi in primavera, e vi nidificano, alcune ne rimangono stazionarie.

II genere Bubola, Upupa trovasi nell'antico continente.

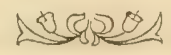




\section{$-93-$ \\ ORDINE VII.}

\section{ALCIONI. ALCIONES.}

Becco lungo, o di lunghezza mediocre, acuto, quasi quadrangolare, dritto, e pochissimo curvo: tarso cortissimo, tre diti d' innanzi, riunite alla base, uno di dietro.

\section{GENERI XXIX}

Merope. Merops. (Linneo).

Carattere generico. Becco mediocre, tagliente, puntato, leggiermente curvo, a lisca elevata, senza intaccatura : narici alla base laterali in forma ovale, aperte, nascoste da peli diretti innanzi. Piedi a tarso corto, tre dita d'avanti, l'esterno saldato alla seconda articolazione del dito di mezzo, e questo alla prima articolazione dell'interno, il dito di dietro largo alla sua base: unghie quella del dito di dietro piu piccola. Ali, primo remigio quasi nullo, il secondo più lungo.

Questi uccelli vivono d'api, e respi, the sanno con destrezza cacciare al volo.

\section{SPEGIE GXXXIII.}

Merops Apiaster (Linneo).

Guépier Vulgaire.

Merope.

Marragau C. M.

Abiolu o Piana C.S.

Fronte d'un bianco tinto di verdastro; nuca ed alto del dorso 
castagno, il rimanente del dorso gialliccio; una fascia nera parte dall'angolo del becco, passa sovra gli occhi, e copre l'orificio delle orecchia, gola d'un giallo d'oro, che termina con una mezza collana nera : parti inferiori d'un verde azzurrognolo.

Di passaggio numerosissimo in aprile, si vedono in tutte le campagne, e particolarmente dove vi sono gli alveari delle api, delle quali fanno gran strage. Nidificano nei buchi scavati in terra, lungo le sponde dei fiumi.

Il genere Merope, Merops trovasi nelle regioni equatoriali dell'antico Continente.

\section{GINER XI}

\section{Alcedine. Alcedo (Limneo),}

Carattere generico. Becco lungo, dritto, angoloso, tagliente, grosso alla base, appuntito, di raro depresso: narici poste ad ambi i lati del becco, e presso alla sua base, forate obliquamente, quasi intieramente chiuse da una membrana ignuda : piedi corti, posti molto indietro del corpo: gambe scoperte: tarso assai grosso, e rotondo: quattro dita, tre d'avanti, l'esterno dei quali saldato a quello di mezzo sino alla seconda articolazione; l'interno non lo è che sino alla prima : uno di dietro molto largo all'origine: unghie grosse, quelle del pollice piu piccole; remigj primo, secondo meno lunghi del terzo, che oltrepassa tutli gli altri.

Volano gli Alcedini con grande rapidità, i loro movimenti sono pronti e risoluti, non possono nè camminare, né arrampicarsi . colgono il loro cibo a pieno volo, spesso a fior d'arequa dopo di arerlo appostato con una pazienza estrema . 


\title{
SPECIE CXXXIV.
}

\author{
Alcedo Ispida. \\ Martin Pecheur. \\ Uccello Pescatore, o di Santa Maria. \\ Pilloni de Santu Perdu C. M. \\ Puzzone de Santu Martinu C. S.
}

Parli superiori d'un verde azzurrognolo marcato sopra la tesla, e coperture delle ali di piccole macchie azzurre turchine: questo colore occupa il centro del dorso, e cuopre tutto il groppone; uno spazio rosso sopra degli occhi con aggiunta d'altro d' un bianco puro: una fascia d'un verde azzurro s'estende dall'angolo del becco insino all'inserzione delle ali; gola, e parte anteriore del collo d'un bianco puro: il rimanente delle parti inferiori d'un rosso di rugine.

Stazionario fra noi, e vi nidifica, frequenta i canali delle saline, e le sponde dei fiumi, e ruscelli. Il principale loro nutrimento ì di pesciolini.

Il genere Alcedine; Alcedo trovasi dappertutto. 


\section{$-9$ \\ ORDINE VIII.}

\section{wis of of \\ CHEIIDONI. CHELIDONES.}

Becco cortissimo, edepresso, assai lungo alla base, mandibolia superiore curva verso la punta; piedi corti; Ire dita d'innanzi intieramente divise, e unite alla base da una corfa membrana : uno dietro, spesso reversibile: unghie assai adunche; ali lunghe.

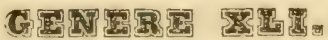 \\ Ronnini Ifirundo (Limeo .}

Carattere generico. Becco corto, triangolare, largo alla base, depresso, l'apertura arriva verso l'occhio: mantibola superiore un poco curva alla punta: narici alla base, oblunghe, fermate in parte da una membrana, sormontate dalle piune sulla fronte: Piedi corti, dita, ed unghie soltili : Ire dita d'avanti, l'esterno unito alla prima arlicolazione del dito di mezzo, un dito di dietro: coda composta di douici penne; ali lunghe, il primo remigio il piu lungo : questi uccelli ci annunziano la bella stagione di primavera, e ci prestano servigi reali con la gran distruzione, che fanno di zanzare, di punteruoli, e di molti altri insetti distruttori dell'agricoltura, $i$ quali inselti si moltiplicano in un paese a misura, che il numero delle rondini, e degli altri uccelli insettivori vi diminuisce; a questo riguardo è bene di risparmiare la polvere e le reti alle rondini, ed agli altri uccelli insettivori. 


\title{
SPECIE CXXXY.
}

\author{
Hirundo rustica (Linneo). \\ Hirondelle de Cheminé. \\ Rondine. \\ Arrundili C. M. \\ Rundine C.S.
}

Fronte, e gola d'un bruno castagno : lutte le parti superiori, i lati del collo, ed una larga fascia sul petto d'un nero a riflessi di violetta ; ventre, e basso-ventre d' un bianco appannato, o rossiccio.

Ai primi di marzo comparisce fra noi in compagnia della seguente, nidifica perfino dentro le nostre case, e partono ai primi di settembre, i giovani partono alcuni giorni dopo.

\section{SPECIE CXXXVI.}

IIirundo Urbica (Linneo).

IIrondelle de fenètre.

Balestruccio.

Arrunditi o Pillomi de Santa Luxia C. M.

Rundine C. S.

Testa, nuca, ed alto del dorso d'un nero a rillessi di color violelta: coda, ali, e grandi coperture delle medesime d'un ners mallo: lutte le parti inferiori, e groppone d'un bianeo purn. 


\section{SPECIE CXXXVII.}

\section{IIirundo Riparia (Linneo).}

IIrondelle de rivage.

Topino.

Arrundili C. 11.

Rundine C. S.

Tutte le parti superiori, le guancie, ed una larga fascia sul petto d'un cenerino bruno, o grigio di soreio: ali d'un bruno nericcio: gola, e parte anteriore del collo, ventre, e coperture inferiore della coda d'un bianco puro.

Arrivano alcuni giorni dopo le prime, e sono le ultime a partire; i nostri pescatori fanno gran strage di queste rondini, massime delle giovani, che prendono nelle canne, che circondano le peschiere principiando l'autunno; per alcuni giorni se ne vedons in piazza a portare in gran quantità dai suddelli.

\section{SPECIE CXXXVIII.}

\section{Hirundo Rupestris (Linneo). \\ Hirondelle de Rocher. \\ Rondine Montana. \\ Arrundili Marina C. M. \\ Rondine Marina C.S.}

Tutte le parti superiori d'un grigio cenerino chiarissimo: gola, parte anteriore del collo, e petto d'un bianco leggiermente tinto di rosso chiaro: ventre, e basso-ventre d'un grigio appannato: tutte le penne della coda hanno nelle barhe interne una macrhia ovale d'un bianco purn, piu grande nelle penne centrali. 
Stazionario, durante l'estate, trovasi nella cosi detta grotta dei Colombi presso Sant'Elia , vive in buona società colla $\mathrm{Co}_{0}$ lumba Aenans, si vede svolazzare insieme nell'interno di questa grotta, nidifica nel medesimo locale.

Ebbi varie volte dei giovani, e vi osservai molte uova rotte. Appena partono le altre suindicate specie, si vedono queste comparire entro la cilta, massimamente quando soffiano i venti di levante, ed allora volano bassissime.

Il genere Rondine, Hirundo, trovasi dappertutto.

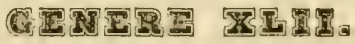 \\ Martinetti. Cypselus (Illiger).}

Carattere generico. Becco molto corto, triangolare, largo alla base, poco apparente, depresso, l'apertura arriva al di sotto dell'occhio : mandibola superiore curva alla punta : narici forate longitudinalmente all' alto del becco presso la lisca, aperte a hordo elevato, guernite di piccole piume : piedi molto corti, le quattro dita dirette innanzi, intieramente divise, dita, ed unghie corte, grosse, e coda composta di dieci penne : ali molto lunghe, primo remigio un po piủ corto del secondo.

I Martinetti sono continuamente in movimento nell'aria, raramente si vedono posarsi in luoghi elevati, non mai peró a terra.

\section{SPECIE CXXXIX.}

Cypselus Alpinus (Temminck).

Martinet à ventre blane.

Rondone di mare.

Varzioni C. M.

Babbarrottu C.S.

Un grigio bruno uniforme i sparso su lulle le parti superiori, 
questo colore forma una larga fascia sul petto, s'estende lungo i fianchi, il basso-ventre, e coperture inferiori della coda, gola e centro del ventre d'un bianco puro.

In aprile compariscono fra noi, frequentano i luoghi montagnosi, nidificano nelle fessure delle rupi, sono comuni, e partono in settembre.

\section{SPECIE CXL.}

Cypselus Murarius (Temminck).

Martinet de Muraille.

Rondone.

Varzia C. M.

Babbarrottu C. S.

Gola d'un bianco cenerino, tutto il rimanente del corpo d'un bruno nericcio, o color di fuligine.

Arrivano annualmente ai primi d'aprile, nidificano nei buchi dei vecchi muri, e sotto le tegole delle nostre case, partono in seltembre.

Il genere Martinetto, Cypselus, trovasi dappertutto.

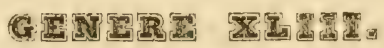 \\ Succracapre. Caprimulgus (Limreo).}

Carattere generico. Becco cortissimo, flessibile, depresso . leggiermente curvo, poco apparente, l'apertura oltrepassa gli occhi : la mandibola superiore curvala alla sua punta, guernita di peli diretli in avanti; narici larghe, alla base fermate da una membrana sormontata dalle piume della fronte: piedi, tre dita d'avanti, e uno di dietro: le dita anteriori riunite da una membrana alla prima articolazione : il dito di dietro reversibile: un- 


\section{- $102-$}

ghie corte, quella del dito di mezzo dentellata a forma di sega, o liscia : coda rotondata, o forcuta, composta di dieci penne: ali lunghe, primo remigio più corto del secondo, che è il piu lungo.

Questi uccelli hanno molto rapporto coi Cipseli, e colle Rondini, questi sono uccelli diurni, e i Succiacapre sono notturni, volano col beceo aperto per cacciare gli insetti notturni, e li Farfalle, Falene, che cacciano al chiaro della luna, alla matlina, al crepuscolo, e verso la sera.

\section{SPECIE CXLI.}

Caprimulgus Europaeus (Limeo).

Engoulevent Ordinaire.

Succiacapre o Notolone.

Avis dellas noces $\mathrm{C}$. $\mathrm{M}$.

Passalitorta C. S.

Il colore generale di quest'uccello è un misculio di punti, di macchie, e di linee transversali, e longitudinali cenerini, giallicci, rossi, e nericci : dei tratti longitudinali neri sono disposti sulla testa, e sul dorso; grandi spazi bianchi sulla gola : una fascia gialla rossiccia traversa l'alto delle ali.

Stazionario fra noi, il nome Sardo del C. II. pare che sia un residuo della lingua Spagnuola, perchè questo nome è pii spagnuolo, che Sardo.

II genere Succiacapre, Caprimulgus trovasi dappertufto. 


\section{$-103-$ \\ OR DINE IX.}

\section{$<i n g=$ \\ COLOMBI. COLUMBAE.}

Becco mediocre, compresso: mandibola superiore coperta alla sua base da una pelle molle nella quale sono forate da narici piu o meno curve verso la punta. Tre dita d'innanzi assai divise; uno di dietro.

\section{GLNER 叉正， \\ Colombi Columbae (Linneo).}

Questo genere è unico dell' ordine, che porta lo stesso nome. Carattere generico. Becco mediocre, dritto, compresso a volta, inclinato verso la punta: base della mandibola superiore coperta d'una pelle molle rigonfia : narici poste alla meti del becco, forate in fessura longitudinale nella pelle molle, che le cuopre: quattro dita intieramente divise, tre d'avanti, ed uno di dietro, articolantesi a livello degli altri : primo remigio piu corto del secondo che è î più lungo di tutti.

I colombi si nutrono di frutti e di semi, vivono ordinariamente a copie nelle selve, impiantansi sulla cima dei maggiori alberi, vi costruiscono i loro nidi che ordinariamente non conIengono che due uova ; rinnovano la deposizione delle uora nel corso dell'anno, ed i due sessi covano alternativamente, i loro pulcini non lasciano il nido, se non quando sono perfettamente in stato di volare. 


\title{
SPECIE CXLII.
}

\author{
Columba Palumbus (Linneo). \\ Columbe Ramier. \\ Colombaccio. \\ Tidoni C. M. \\ Tudone C. S.
}

Su i lati del collo e su $i$ hordi delle ali un grande spazio bianco; testa, tempia, gola, groppone, e parte superiore della gola d'un cenerino azzurrognolo; petto ed alto del ventre d'un bel color vinato, ma a riflessi cangianti su i lati del collo: dorso, ed ali d'un cenerino bruno: ventre, e bassn-ventre d'un cenerino bianchiccio.

Stazionario in tulte le parti dell'Isola, è comunissimo: nell'inverno se ne prendono in maggior quantita; si nutre a preferenza in questa stagione di ghiande.

\section{SPE EIE CXLIII.}

Columba OEnas.

Colombe Colombin.

Colombello.

Succella C. M.

Tudone C. S.

Testa, gola, ali, e parti inferiori d'un azzurro cenerino : lati del collo d'un verde cangiante: petto di color feccia di vino; alto del dorso d'un cenerino bruno: gropponed'un cenerino azzurrognolo.

Di passaggio annuale in autunuo, parte principiando l'estate, se ne fa preda in gran numero, come del precedente. 


\title{
SPECIE CXLIV.
}

\author{
Columba Livia (Brisson). \\ Colombe Biset. \\ Piccione Terrazzolo. \\ Columbu Aresii C. M. \\ Colombu Aresle C. \$.
}

Parti superiori, ed inferiori d'un azzurro cenerino: lati del collo d'un verde cangiante; groppone d'un bianco puro: delle fascie transversali nere sulle ali.

Fanno stazioni fra noi, non abbandonano mai le rupi, e le grotte massime quella di Sant'Llia la quale porta il nome di grotta dei Colombi.

\section{SPECIE CXLV.}

Columba Turtor (Linneo).

Colombe Tourtorelle.

Tortora.

Turturi C. M.

Turture C. S.

Testa, e nuca d'un cenerino vinato; sui lati del collo uno spazio composto di piume nere terminate di bianco : parte anteriore del collo, petto, od alto del ventre d'un color di vino chiaro: dorso d'un bruno cenerino: bordo delle ali d'un cenerino azzurrognolo; ic altre coperture d'un rosso di rugine con una macchia nera nel centro delle piume; basso-ventre, e coperture inferiori della coda d'un bianco puro.

Di passaggio periodico annuale in primavera, vi nidifica, $c$ parte in seltembre.

Il genere Colombo, Columbae trovasi dappertutto. 


\section{ORDIVE $\mathrm{X}$.}

\section{GALLINACCI. GALLINAE.}

Becco corto, convesso, talvolta coperto d'una cera : mandibolit superiore piu o meno curva alla sua base, o verso la punta soltanto. Narici laterali coperte d'una membrana a volta, nuda, oppure wnernita di piume. Tarso allungato; tre dita innanzi riunite da uni membrana, uno di dietro che si articola piu alto degli altri, falvolta piccolissimo, od anche interamente obliterato.

Notn. Il genere 45, 46, 47, 48. Gallopavo, Fagiano, 'Tetraone, Pterocle' manca affatto fra noi.

\section{GENIRE XIX.}

\section{Pervici. Perdix (Latham).}

fiarallere generico. Becco corto, compresso, forte, a base nuda: mandibola superiore vuota, convessa, molto curva verso la punta : narici alla base, laterali coperte a meti da una membrana vuota e nuda. Piedi, tre dita d'avanti, e uno di dietro, i primi riuniti da una membraua alla prima articolazione. Coda composta di 1't o 18 penne, corta e rotondata, piegata verso la terra. Ali corte, i tre primi remigj sc no i piu corli, il quarto ed i! yuinto sono i più lunghi, e tante volte il puinto remigio é piu lungo di tutti. Questi uccelli si moltiplicano di molto nei climi temperali, e caldi, ove vivono stazionarj.

Volte. La prima sezione Francolino manea fira noi. 


\section{Sieconon Sizzinne.}

Pernici (propriamente delle).

Queste hanno il tarso munito d'una callositi, o intieramente liscio.

\section{SPECIE CXLVI.}

Perdix Petrosa (Latham:

Perdrix Gambra.

Pernice.

Perdixi C. M.

Perdighe, e Perdya C. S.

Fronte, alto della testa, e nuca di castagno carico : si dilata sui lati del collo in una larga collana, che si restringe per d'avanti, su quesla collana son delle macchie bianche: piume delle orec(hie rosse: gola, tempia, ed una larga fascia sopra degli oechi di un cenerino azzurognolo : pelto cenerino: ventre rosso: sulle piume cenerine dei fianchi, ha una larga fascia transversale bianca, e rossa, bordate ai lati d'una stretta fascia nera : sono terminate di rosso.

Stazionaria, c comunissima in tutte le stagioni dell anno, lanto nei luoghi montuosi, che in pianure.

\section{Coge. \\ Cerza Sezinne.}

\section{(2)AGLIA.}

Queste hamo la corla molto corta, e piegata verso la terra. i nascosta dalle piume del groppene, il primo remignio delle ali è il pii lungo. 


\title{
SPECIE CXLVII.
}

\author{
Perdix Coturnix (Latham). \\ La Caille \\ Quagtia. \\ Quallia o Circuri C. M. \\ Trespotres C. S.
}

Sommita della testa variata di nero, e rossicio con tre fascie longitudinali, due delle quali sopra degli occhi, ed una in mezzo della testa: parti superiori d' un cenerino bruno, con delle macchie nere, e fascie gialliccie: del rosso sulla gola, contornato di due fascie d'un bruno nericcio: parti inferiori del collo, petto, e fianchi d'un rosso chiaro con delle striscie longitudinali bianche.

Fa pure stazione fra noi, ed i comune come la precedente.

II genere Pernice, Perdix, trovasi dappertutto.

Nota. Il genere 50 Hemipodius manca fra noi.

\section{OR INE XI.}

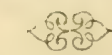

\section{ALETTORIDI. ALECTORIDES.}

Becco largo come la testa, o piú corto, robusto, e duro: mandibola superiore curva, convessa : ordinariamente alunca verso la punta. Tarso lungo, ed esile; tre dita innanzi, uno di dietro. articolato piu alto degli altri. 


\section{GENERE II:}

\section{Glareola. Glareola (Brisson).}

Carattere generico. Becco piu corto della testa, robusto, convesso, compresso verso la punta: mandibola superiore curva nell'ultima estremitá della sua lunghezza, l'inferiore dritta, narici poste ai lati, e presso la base del becco obliqui: piedi piumati sino al ginocchio: tarsi lunghi, e gracili : quattro dita, tre d'avanti, dei quali il medio unito all'esterno mediante una membrana netta : pollice articolato sul tarso, e posando a terra sulla punta: unghie strette a lesina: ali lunghissime : il primo remigio oltrepassante tutti gli altri. Le Glareole mostrano nel corso altrettanta agilita quanta hanuo leggerezza nel volo; quindi veggonsi pigliare con destrezza veramente ammirabile le picciole prede, che corrono sulle sabbie, come quelle, che volteggiano tra i giunchi e le canne in cerca dei piccoli inselli acquatici.

\section{SPECIE CXI.VIII.}

Glareola Torquata (Meyer)

Glareole a Collier

Pernice di mare.

Perdixi de mari C. M.

Perdighe marina C. S.

Sommita della testa, nuca, dorso, scapolari, e coperture dell' ali d'un grigio bruno: gola, e parte anteriore del collo d'un bianco leggiermente tinto di rossiccio: questo colore è come incominciato da una strettissima fascia nera, che rimonta verso l'angolo del beces: spazio tra l'occhio, ed il becco nero: petto, d'un brumo hianchicero: parti inferiori d'un bianco accordato col rossiccio. 
Di passaggio in primarera, ma non annuale. Frequenta le sponde del mare, e le guincaglie ove varie volte la cacciai.

II genere Glareola, Glareula si trova solamente nell' antico continente.

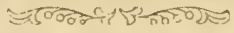 \\ O R D I NE XII.}

ac. - ;

\section{CORRIDORI. CURSORES.}

Becro mediocre, o corto: piedi lunghi, nudi sopra il ginoc'chio: due, o tre dita soltanto dinanzi, nessuno dietro.

\section{GINER III. \\ Ottarde, Otis (Limneo).}

Carallere generico. Becco lungo come la testa al più, dritto, conico, ('ompresso lateralmente: mandibola superiore alquanto curva verso la punta, oltrepassante l' inferiore, che cuopre coi suoi hordi: Narici ovali, situate verso il mezzo del becco: approssimate tra loro e coperte, piedi lunghi, nudi sopra il ginocchio: tre dita all' innanzi, corte, unite alla base, e bordate da membrane senza pollice: ali mediocri, il primo remigio di lunghezza media, il secondo un po' più corto del terzo che è il piu lungo. Le Ottarde sono molto piu atte alla corsa, che al volo, quando sono costrelte ad abhandonarsi a quest' ultimo uso delle loro facolta, sembrano farlo con timore, e piu che é possibile vicino alla superficie della terra, chetuttavia serorono a basen volo 
con gran rapidita. Dimorano constantemente nelle grandi pianure coperte di messi, e nei cespugli meno frequentati. Il loro nutrimento consiste in semi, erbe tenere, ed inselti. L'Ottardi è un selvaggiume assai ricercato.

Prima Sezione, le mandibole compresse alla base.

\section{SPECIE CXLIX.}

Otis Tetrax (Linneo).

Outarde Canepetiere

Gallina Pataiuola o Fagianella.

Faggianu o Pidraxiu C. M.

Giaddina de campu o Pudda campina C. S.

Sommita della testa, e nuca d'un gialliccio chiaro con delle macchie brune; lati della testa, e parte anteriore del collo d'un cenerino opaco ; questo colore è cinto da una collana in forma di croce di Sant'Andrea d'un bianco puro: tutto il basso del collo coperto di piume d'un nero profondo, che sono piu luughe sulla nuca: il pelto contornato da una larga fascia bianca susseguita da un altra piu strelta nera: il rimanente delle parti inferiori, il bordo delle ali, e le coperture superiori della coda d'un bianco puro: le parti snperiori d' un gialliccio chiaro con numerosissimi zigzag nericci, e qualche grande macchia nera.

Fa stazione fra noi, e vi fa il suo nido. Abita in tutte le pianure arride.

Il genere Ottarda, Olis trovasi nelle regioni calde dell'antico continente.

La seconda Sezione colle mandibole depresse alla lorn base, manca fra noi

Nola. II genere 53 Cursorius, manca fra noi. 


\section{$-112-$ \\ O R D I NE XIII.}

\section{GRALLE. GRALLATORES.}

La forma del becco assai varia, talrolta a cono lunghissimo, piu spesso dritto e compresso : di raro schiacciato. Piedi lunghi, gracili, piu o meno nudo sopra il ginocchio.

\section{GENERE}

Edciemo. OEdicnemus (Temminck!.

Carattere generico. Becco piu lungo della testa, dritto, forte, un po' depresso alla base, compresso verso la punta ; spigolo della mandibola superiore elevata ; mandibola inferiore formante l'angolo; narici doppiamente contorte, situate verso il mezzo del becco, fesse longitudinalmente sino alla parte cornea, aperte d'avanti e forate da parte a parte. Piedi lunghi, gracili, tre dita anteriori provviste d'una membrana che le unisce sino alla prima articolazione, nessun dito posteriore o pollice, ali di mediocre lunghezza, il primo remigio un po' piu corto del secondo che oltrepassa tutti gli altri; rettrici fortemente scaglionate. Gli Edicnemi sono uccelli che frequentano le parti elevate e deserte dei terreni aridi e sabbionicci; queste sono le loro abitazioni favorite: sono di naturale sommamente timido, ed anche selvaggio. 
Il loro nutrimento consite in lumache, insetti, e piccoli rettili. Il loro volo va ordinariamente accompagnato da grida acute, ed in certo modo lamentevoli, che si fanno udire da lontano.

\section{Erima Bivisione}

Questi mancano sempre del dito posteriore ossia pollice.

\section{SPECIE CL.}

Oaedionemus Crepilans Temminck:

Oaedicneme Criard.

Occhione.

Pudda media C. $\mathrm{M}$.

Pudda areste, Purldighinu, e Ziriolu C. S.

Tutte le parti superiori d'un rossiccio cenerino con una macchia longitudinale in mezzo a ciascuna piuma : spazio tra l'occhio ed il becco, gola, ventre, e coscie d'un bianco puro: collo, e pelto leggiermente colorito di rossiccio, e seminato di tratti longiludinali bruni : sulle ali una lunga fascia bianca.

Stazionario fra noi, e vi nidifica.

Il genere Edicnemo, Oaedicnemus trorasi solamente nell' antico Continente.

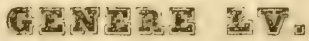 \\ Savderlivg. Calidris (Illiger).}

Carattere generico. Becco mediocre, gracile, dritto, flessibile in tutta la sua lunghezza, compresso dopo la base, depresso alla punta, schiacciato, e piu largo che nel mezzo ; il solco nasale prolangato verso la punta. Narici laterali, aperte Inngiludinalmente: 
Piedi sottili, tre sole dita dirette in avanti, quasi intieramente divise, ali mediocri; il primo remigio è il piủ lungo. Questi uccelli si nutrono di piccoli insetti marini che cercano lungo il bordo del mare.

\section{SPECIE CLI.}

\section{Calidris Arenaria (1lliger).}

Sanderling variable.

Calidra.

Zurrulioltu C. M.

Zirriolu C. S.

Tutte le parti superiori ed i lati del collo d'un cenerino bianchiccio, con un piccolo tratto piu carico nel mezzo di ciascuna piuma: faccia, gola, parte anteriore del collo, e tutte le parti inferiori d'un bianco puro; cintura, e bordo delle ali, e remigj neri.

Nidifica fra noi, ed è comunissimo, si vede a torme lungo le sponde del mare particolarmente nei luoghi sabbiosi.

Il genere Sarderling Calidris trovasi dappertulto.

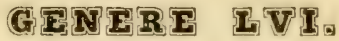

\section{Trampoliere Himastopus (Brisson).}

Carattere generico. Becco lungo, delicato, cilindrico, appiatlito alla base, compresso alla punta: mandibole scanalate longitudinalmente fino alla metá della loro lunghezza : narici laterali, lineali, lunghe; piedi molto lunghi, gracili ; tre dita dirette in avanti, quello di mezzo riunito al dito esterno da una larga membrana, l'interno da un piccolo rudimento. Unghie molto piccole, piatte: ali lunghissime, primo remigio oltrepas- 
sante tutti gli altri. Questi uccelli frequentano di preferenza le sponde dei mari, ed i laghi saliniferi, che quelli d'acqua dolce. Si nutrono di vermicini, e piccole mosche.

\title{
SPECIE CLII.
}

\author{
IImantopus Melanopterus (Meyer). \\ Echasse a Manteau noir. \\ Cavaliere d'Italia. \\ Zurruliu peis longus C. M.
}

Faccia, collo, petto, e tutte le parti inferiori d'un bianco puro, questo prende una leggriera tinta rossa sul petto, e ventre; occipite, e nuca, neri, o nericci con delle macchie bianche; dorso, ed ali d'un nero a riflessi verdastri : coda cenerina .

Di passaggio annuale in autunno, e parte principiandol'estate. II genere Trampolo, Himantopus trovasi dappertutlo.

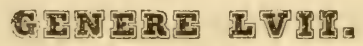

\section{Ostrichiere. Haematopus (Linneo).}

Carattere generico. Becco robusto, drilto, lungo, compresso; mandibole eguali, uniformi ; narici oblunghe, poste in una scanalatura da ogni parte del becco; piedi forti; tarsi mediocremente elevati; tre dita direlte all'innanzi, il medio unito fino alla prima arlicolazione coll'esterno mediante una membrana, e coll'interno mediante un semplice rudimento, tutli rivestiti d'un rudimento somigliante, senza dito pollice; ali mediocri, la prima penna remigia la più lunga.

Gli Ostrichieri vivono soli, o riuniti a piccole torme, sono 
continuamente occupati a scavare nella sabbia, intorno alle rocche battute dal flusso e riflusso delle onde per scuoprirvi le conchiglie bivalvi, di cui si nutrono esclusivamente. Le grida acute, che fanno questi uccelli all'avvicinarsi dell'uomo, divengono per gli altri uccelli segnale d'imminente pericolo, per cui non di raro il cacciatore deluso nella sua speranza uccide l'Ostricchiere indiscreto, che in ogni altro tempo avrebbe risparmiato per la sua carne fetida, e stomachevole.

\section{SPECIE CLIII.}

\section{Haematopus Ostralegus (Linneo). \\ Huiterier. \\ Ostrichiere, o beccaccia di mare. \\ Beccaccia de mari C. M.}

Testa, nuca, alto del petto, dorso, ali, ed estremità della coda d'un nero profondo: una ben distinta gorgiera sulla gola, il groppone, fascia transversale delle ali, e tutte le parti inferiori d'un bianco puro. In scarso numero trovasi fra noi questa specie, ma se ne fa preda in tutte le stagioni, frequentano le spiaggie, e gli scogli.

Il genere Ostricchiere Haematopus trovasi dappertutto.

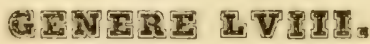 \\ Piviere, Cimaradrius (Limeo).}

Carattere generico. Becco piu corto della testa, gracile, dritto, rompresso, solco nasale prolungato fino ai due terzi. Le mandi- 
bole rigonfie verso la punta, narici alla hase, intaccate, aperte longitudinalmente nel mezzo di una gran membrana, che ricuopre la fossetta nasale: piedi lunghi, o di mediocre lunghezza, sottili, tre dita dirette in avanti, l'esterno riunito a quello di mezzo da una corta membrana, il dito interno diviso: coda leggiermente ritondata, o quadrata: ali, il primo remigio un po' piủ corto del secondo, che è il piu lungo. Questi uccelli compariscono sempre in numerose truppe, frequentano i luoghi umidi, e le terre fangose dove cercano il loro alimento, che consiste in vermi, ed insetti acquatici.

\title{
SPECIE CLIV.
}

\author{
Charadrius Pluvialis (Linneo). \\ Pluvier dorẻ. \\ Piviere dorato. \\ Culingioni de terra C. M. \\ Culurzone de terra C.S.
}

Sommità della testa, e tutte le parti superiori del corpo, delle ali, e della coda d'un nero di fuligine, marcato da grandi macchie d'un giallo d'oro disposte sui bordi delle barbe: lati della testa, collo, e petto variati da macchie cenerine, brune, e gialliccie: gola, e parti inferiori bianche.

Di passaggio annuale in autunno, e parte principiando l'estate. 


\title{
S PECIE CLV.
}

\author{
Charadrius Morinellus (Linneo). \\ Pluvier Guignard, \\ Piviere Tortolino. \\ Zurruliu conca de molenti.
}

Sommità della testa, ed occipite d'un cenerino nericcio: le larghe sopracciglia d'un bianco rossiccio si riuniscono sull'occipite: faccia bianca punteggiata in nero: parti superiori d'un cenerino nericcio tinto di verdognolo: tutte le piume di queste parti incornicciate di rosso: petto e fianco d'un cenerino rossiccio : il largo cinturone del ventre, ed il centro del ventre d'un bianco puro.

Arriva in autunno, e parte in primavera.

\section{SPEGIE CLVI.}

Charadrius Hiaticula (Linneo).

Grand Pluvier à Collier.

Corriere grosso.

Zurruliu peis arrubius C. M.

Ziriolu C. S.

Fronte, spazio tra l'occhio, ed il becco, una larga fascia coronale che passa sopra gli occhi, e si dirige verso l'occipite, un gran cinturone sul petto, le di cui estremità si uniscono alla nuca, tutto d'un nero profondo: fronte, gola, e tutte le parti inferiori d'un bianco puro: occipite, e tutte le parti superiori d'un bruno cenerino: Ire quarti del becio rossi, l'altro nero. 
Comune, e vi nidifica. Vedesi lungo il litorale del mare parvicolarmente passata la Scaffa.

\title{
SPEGIE CLVII.
}

\author{
Charadrius minor (Meyer). \\ Petit Pluvier à Collier. \\ Corriere piccolo. \\ Zurlioteddu C. M. \\ Ziriolu pitiu C. S.
}

Fronte, spazio, tra l'occhio, ed il becco, ed una larga fascia coronale, che passa sopra gli occhi, e si getta in linea dritta al di sotto, ed uno stretto cinturone, le di cui estremità si uniscono alla nuca d'un nero profondo, un bianco puro cuopre la fascia frontale, la gola, una collana, e le parti inferiori, occipite, e tulte le parti superiori d'un bruno cenerino: il becco intieramente nero.

Comune, stazionario, frequenta i luoghi paludosi, e le caselle delle saline.

\section{S PECIE CLVIII.}

Charadrius Cantianus (Latham).

Pluvier ä Collier interrumpu.

Frattino.

Zurruliu conca de molenti C. M.

Ziriolu C. S.

Fronte, larghe sopracciglia, una fascia sulla nuca, e tutte le parti inferiori d'un bianco puro: spazio tra l'occhio, ed il becco, ed un grande spazio angolare sulla testa, ed una larga macchia 
da ciascun lato del petto, d'un nero profondo: una grande macchia d'un nero cenerino dietro l'occhio : testa, e nuca d'un rosso chiarissimo ; parti superiori d'un cenerino bruno.

Molto più comune dei precedenti, nidifica nelle nostre spiaggie.

Il genere Piviere, Charadrius, Irovasi dappertutto.

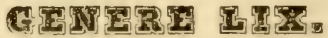 \\ Vanello. Vanelius (Brisson).}

Carattere generico. Becco corto, gracile, dritto, compresso, punta delle due mandibole rigonfie: base della mandibola superiore molto dilatata dal prolungamento del solco nasale : narici laterali, aperte longitudinalmente nella membrana, che cuopre il prolungamento ; piedi gracili, tre dita d'avanti, ed uno dietro, il dito di mezzo riunito all'esterno per mezzo d'una corta membrana, quello di dietro quasi nullo, o molto corto, articolato sopra il tarso, non toccante a terra: ali larghe, o accuminate, primo remigio il più lungo, il terzo remigio d'uguale grado, o piu corto del quarto e del quinto, che sono i piu lunghi.

I vanelli frequentano i luoghi bagnati d'acque dolci, e salamastre, ed i prati umidi, si nutrono di vermi di terra, e di larve.

\section{Seconda Divisione}

\section{Gralle a quattro dita.}

Questi hanno sempre un pollice distinto, os'appoggia a terra in tulta la sua lunghezza, o s'articola sopra il tarso. e non porta a terra che l'unghia. 


\section{\#liuta Sezione.}

Il primo remigio dell'ala è il più lungo.

\section{SPECIE CLIX.}

Vanellus Melanogaster (Bechstein).

Vanneau.

Pivieressa.

Curruliu Brenti niedda C. M.

Ziriolu C. S.

Fronte, gola, centro del ventre, coscie, basso-ventre, e coperture superiori della coda d'un bianco puro: sopracciglia , parte anteriore del collo, lati del petto, e fianchi d'un bianco variato da macchie cenerine, e brune : parti superiori d'un bruno nericcio variato da macchie d'un giallo verdognolo, ma tutte le piume terminate da cenerino, e bianchiccio: lunghe penne interne delle ali d'un nero profondo, coda bianca, ma rossiccia verso la punta.

Arrivano fra noi in aprile: un individuo di questa specie in perfetta livrea fu da me cacciato in maggio del 1838 verso la Maddalena, esiste nel nostro Museo.

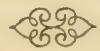

\section{Secontia Sezione.}

I tre primi remigii esteriori egualmente gradati, il quarto, ed il quinto sono i piu lunghi. 


\title{
$-122-$
}

\section{SPEGIE CLX.}

\author{
Vanellus Cristatus (Meyer). \\ Vanneau Huppé \\ Pavoncella o Fita. \\ Lepuri de Argiola C. M. \\ Lepere de Arzola C.S.
}

Piume occipitali lunghissime, affilate, e ricurvate in alto: sommità della testa, ciuffetto, parte anteriore del collo, e petto d'un nero a riflessi : parti superiori d' un verde oscuro a riflessi risplendenti : lati del collo, ventre, basso-ventre, e base della coda d'un bianco puro.

Stazionario fra noi, e vi nidifica.

Il genere Pavoncello, Vanellus, trovasi dappertutto.

\section{GENERE Z是。}

Volta pietre. Strepsilas (Illiger).

Carattere generico. Becco mediocre, duro alla punta, forte, dritto, di forma conica, allungato, leggiermente curvo in alto: a lisca appiatata, la punta dritta troncata : narici laterali poste alla base, lunghe, fermate a meta da una membrana, forate da parte a parte: piedi mediocri, piccola nuditá al di sopra del ginocchio: tre dita d'avanti, e uno di dietro, tre dita anteriori unite alla base da una cortissima membrana, il posteriore articolato sopra il tarso. Ali accuminate il primo remigio il piu lungo di tutti. 
Questi uccelli hanno l'abitudine di voltare le pietre con gran destrezza, per cercarvi il loro nutrimento, che consiste in piccole conchiglie bivalve, e scarabei marini.

\section{SPECIE CLXI.}

Strepsilas Collaris (Temminck).

Tourne-pierre ả Collier.

Volta pietre.

Zurruliu C. M.

Fronte, spazio tra il becco, e l'occhio, una larga collana sulla nuca, una parte del dorso, una fascia longitudinale, ed un'altra transversale su l'ala, coperture superiori della coda, centro del ventre come le altre parti inferiori d'un bianco puro: una stretta fascia frontale nera, che passando d'avanti gli occhi si dilata al di sotto, ove da una parte si dirige sulla mandibola inferiore, e dall' altra dilatandosi di nuovo sui lati del collo circonda la gola , e forma una larga piastra sulla parte anteriore del collo, e sui lati del pelto : sommità della testa d'un bianco rossiccio a tratti longitudinali neri : alto del dorso, scapolari, e coperture delle ali d'un rosso castagno vivo, seminato irregolarmente di grandi macchie nere: una larga fascia bruna sul groppone.

Di passaggio in marzo, ed aprile, ne feci preda d'un individuo maschio in perfetta livrea, all'imboccatura della Scaffa: esiste nel nostro Museo.

Questo genere è composto di questa sola specie, e trovasi dappertutto. 


\section{GLNER2 2XI \\ Grue, o Gru. Grus (Bechstein).}

Carattere Generico. Becco lungo, o più lungo della testa, robusto, dritto, compresso, a cono allungatissimo, ma ottuso verso la punta, mandibola fortemente scanalata ai lati, e presso la base : spicolo elevato: narici poste alla metà del becco, chiuse di dietro da una membrana: regione degli occhi, e base del becco ordinariamente nude, e coperte di capezzoli ; piedi lunghi, e forti, piumati molto sopra il ginocchio : quattro dita, dei quali tre d'avanti, l'esterno riunito al medio, mediante un rudimento di membrana, l'interno diviso, il pollice articolantesi assai in alto sul tarso: ali mediocri, il primo remigio più corto del secondo, e questo uguagliando alle volte il terzo, che è il piu lungo. Le Grù fra tutti gli uccelli viaggiatori, sembrano quelle, che mettono maggiore previdenza nei loro trapassi rapidi dalle regioni boreali alle contrade equatoriali, e nei ritorni periodici da queste contrade verso quelle, che precedentemente aveano lor fatto abbandonare i pericoli d'una fame totale. Intraprendono i loro viaggi a truppe numerose. Si alimentano di rane, lucertole, insetti, erbe, e semi.

\section{SPECIE CLXII.}

Grus Cinerea (Bechstein).

Grue Cendrée.

Gru o Grue.

Grui C. 11.

Grue C. S.

Su tutte le parti del corpo del grigio cenerino: parte anteriore 
del collo, ed occipite d'un grigio nericcio opaco : fronte, e spazio tra l'occhio, ed il becco guernito di peli neri : sommitá della lesta nuda, e rossa.

Di passaggio in primavera, e in autunno, frequentano i campi, ed i luoghi umidi; se ne fa preda nelle due suindicate stagioni.

Un bellissimo individuo ne fu cacciato dal signor Professore Ugo, il quale ne fece graziosamente dono al nostro Museo, dove tuttora esiste.

Il genere Gru, Grus, trovasi dapperlutto, eccetto in Oceania.

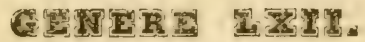

Cicogna. Ciconia (Brisson).

Carattere generico. Becco lungo, dritto, cilindrico, conico, aguzzo, tagliente, compresso lateralmente, d'eguale altezza della testa, talvolta un poco curvo in alto: mandibola superiore a spicolo rotondato a solchi obliterali ; narici longitudinali, lineari, collocate presso la base del becio : occhi circondati da uno spazio nudo, che talvolta s'estende sopra la faccia, senza però comunicare col becco : piedi lunghi : quattro dita, tre d'avanti congiunte da una membrana sino alla prima articolazione, uno di dietro posante a terra sopra piu falangi, unghie corte, depresse senza dentelli : ali mediocri, il secondo remigio piu lungo del primo, e più corto del terzo, e del quarto, e del quinto, che sono i piu lunghi.

Le Cicogne sono uccelli di gran volo, capaci d'imprender viaggi di lungo corso, quindi se ne incontrano in tutte le contrade dove possono i rettili loro somministrare abbondante alimento. Il bisogno di questo cibo le trasporta in due periodi dell'anno verso lunghi opposti ; con tali emigrazioni formansi 
una temperatura quasi constantemente eguale a fine d'evitare la stagione in cui i rettili colpiti da letargia, rimangono assiderati, e nascosti una parte dell'anno. Egli è pure questo cibo di cui fanno gran consumo, che loro procurò appo tutti i popoli, non solo un semplice affetto, ma una protezione religiosa. Le nazioni più egoiste, al pari delle più generose, le più selvaggie, come le piu incivilite, alla voce obbedendo dell'interesse, od a quella della gratitudine, hanno coll'uso di sovente anche con articoli dei loro Codici sanzionata l'accoglienza protettrice fatta ad uccelli, aiquali vanno obbligati del servizio di purgare il suolo da quell'immensa quantita di rettili, che minacciava per la facile riproduzione, e per la longevità di cuoprirlo intieramente.

\section{SPECIE CLXIII.}

Ciconia alba (Bellon).

Cicogne blanche.

Ciconia bianca.

Ciconnia C. II.

La testa, il collo, e tulte le parti del corpo d'un bianco puro, scapolari, ed ali nere: becco, e piedi rossi.

Di passaggio accidentale.

\section{SPECIE CLXIV.}

Ciconia nigra (Bellon).

Cicogne noire.

Ciconea nera.

Ciconnia C. M.

Testa, collo, e tutte le parti superiori del corpo, le ali, e lis 


\section{$-127$}

coda nericci a riflessi porporini, e verdastri : parte inferiore del petto, e ventre d'un bianco puro, pelle nuda degli occhi, e quella della gola d'un rosso cremisi.

Di passaggio piu frequente della precedente.

Il genere Ciconia, trovasi dappertutto.

\section{GENERE LXIXI}

\section{Arrone. Ardea (Linneo).}

C'arattere generico. Becco piu lungo, oppure della stessa lunghezza della testa, conico, schiacciato, appuntato: mandibole ad orli taglienti, la superiore dritta, o leggiermente curva, debolmente scanalata colla lisca rotonda: narici collocate a ciascun lato del becco, o quasi alla sua base, fesse longitudinalmente in una scanalatura, e mezzo coperte da una membrana : uno spazio nudo da ciascuna banda del becco in mezzo al quale stanno gli occhi ; piedi lunghi, e gracili, quattro dita, tre d'avanti, il cui interno congiunto all'intermedio per mezzo d'una piccola membranetta, e l'interno libero; il pollice articolantesi sulla faccia interna del tarso, ed a livello degli altri : unghie lunghe, poco arcuate, compresse, acute, quella di mezzo dentellata interiormente; primo remigio quasi tanto lungo quanto il secondo, ed il terzo, che sorpassano tutti gli altri. Questi uccelli sono dotati d'organi proprj ad attraversare immense estensioni aeree d'una sobrieta che fa loro sopportare lunghe astinenze, parendo inoltre che sostengano senza soffrire le alternative dei termini opposti della temperatura atmosferica. Non è meraviglia se le Ardee passano facilmente da un clima all'altro, e pervengono anzi cosi a fare il giro del mondo. Col corpo immobile, e perpendicolare 
sopra gambe rigide, il collo piegato sul petto, la testa conficcata nelle spalle, attendono per ore, ed ore intiere, e nel medesimo atteggiamento, che si presenti ai loro occhi alcuna preda, sulla quale slanciare rapidamente quel becco lungo, ed appuntito.

Preferiscono assai generalmente i pesci, ma in difetto d'un tal cibo, contentansi dei rettili, ed anche d'anelidi, e molluschi.

\section{Ylima Seziolte.}

\section{Amoni (propriamente detti).}

Hanno il becco più lungo della testa, tanto largo, o piu largo, che alto alla base, mandibola superiore presso a poco dritta: una grande porzione del Tibia nuda.

\section{SPECIE CLXV.}

Ardea Cinerea (Latham).

Heron cendrè.

Airone cenerino o Nonna.

Menga C. M.

Perdezornadas C. S.

Alla parte posteriore della testa delle lunghe piume affiate nere: simili d'un bianco lustro pendono sul basso del collo: quelle similmente allungate degli scapolari sono d'un cenerino argentino: fronte, collo, centro del ventre, bordo delle ali, e coscie d'un bianco puro: occipite, lati del petto, e franchi d'un nero profondo: delle grandi macchie longitudinali nere, e cenerine sulla parte anteriore del collo: dorso, ed ali di un cenerino azzurro chiarissimo. 


\title{
$-129$
}

Di passagggio annuale in autunno, e partono alla fine di maggio, alcune anche più tardi. Abitano i luoghi paludosi.

\section{SPECIE CLXVI.}

\author{
Ardea purpurea (Linneo). \\ IIéron Pourpré. \\ Airone Porporino o Ranocchiaja. \\ Menga arrubia C. M. \\ Perdezornadas C.S.
}

Delle lunghe piume affilate d'un nero verdastro sulla parte posteriore della testa: simili d'un bianco porporato sul basso del follo; ed altre d'un brillantissimo rosso di porpora sugli scapolari ; sommiti della testa, ed occipite d'un nero a riflessi verdastri ; gola bianca, parti laterali del collo d'un bel rosso; tre stretlissime fiscie longitudinali rosse, nere, e porporine sulla parte anteriore del collo : dorso, ali, e coda d'un cenerino rossiccio a riflessi verdastri ; coscie e basso-rentre rossi : fianchi e petto d'un colore di porpora risplendente.

Di passaggio come la precedente, ma meno comune.

\section{SPEGIE CLXVII.}

Ardea Egrelta (Linneo).

IIéron Aigrette.

Airone Maggiore bianco.

Garza manna, o Menga bianca C. M.

Cau Marinu C. S.

Tutte le piume d'un bianco puro; sulla testa un piccolo ciuffefto pendente: alcune piume del dorso lunghe d'un piede e 
mezio, a bacchetta forte, e dritta con delle lunghe barbe rare, ed affilate.

Di passaggio come la precedente.

\title{
SPECIE CLXVIII.
}

Ardea Egrelloides (Temminck).

Herron Aigrettoide.

Garza bianca.

Garza bianca C. M.

Del bianco puro dappertutto, non esclusi li piumini del dorso, ed il mazzo di piume filamentose del basso del collo.

Ebbi per la prima rolta un individuo maschio di quesla specie ai 27 dicembre 18 1 1 preso nei nostri stagni, il quale combina con l'esalta descrizione del signor Temminck nella quarta parte del suo Mamuel d'Omilhologie, altra differenza non trovo che sulla lunghezza totale, che invece di pollici 26 a 27 il mio è di pollici 25 e 7 linee. Un altro individuo maschio l'ebbi il 17 gennajo $18 \% 2$, il quale era già restito dal mazzetto di piume al basso del collo, come anche di quelle del groppone; trovansi deposti nel nostro Museo.

\section{SPEGIE CLXIX.}

\author{
Ardea Garzelta (Linneo). \\ Hèron Garzette. \\ Airone Minore o Garella. \\ Garza bianca C. M. \\ Can Marimu C. S.
}

Tutte le piume d'un bianco puro: all'oscipite un ciufleto 


\section{- $131-$}

pendente formato da due, o tre piume lunghe, estrette; un gran mazzo di piume simili streltissime, e lustre sul basso del collo ; sull'alto del dorso nascono tre ranghi di piume lunghe da sei ad otto pollici a bacchetta debole, contorniate, e rialzate verso la punta queste piume portano le barbe rare, morbide, ed affilate.

Arrivano fra noi ai primi di settembre, alcune si redono tutto l'anno.

\section{Sarention Sezione.}

Butori.

A becco lungo, come la testa, o poco piu lungo, piu alto che largo, schiaccialissimo; mandibola supcriore leggiermente curvata; una piccola porzione del Tibia nudo.

\section{SPECIE CLXX.}

Ardea Stellaris (Linneo).

Héron grand butor.

Tarabuso.

Caboni de Canna C. MI.

Corvu Ambidastru C. S.

Larghi mostacchi, ed alto della testa neri, tutto il fondo delle piume d'un rosso gialliccio chiarissimo, marcato sui lati del collo da zigzag bruni, e sulla parte anteriore del medesimo da macchie brune, e rosse; sulle parti inferiori delle grandi striscie nere, e longitudinali; sull'alto del dorso molto nero nel centro delle piume; sulle coperture delle ali dei zigzag neri, e bruni.

Comunissimo fra noi, arrivano in autunno, e partono alla fine di magggio. Abita i luoghi paludosi in mezzo alle canne. 


\section{$-132-$}

\section{SPECIE CLXXI.}

Ardea Ralloides (Scopoli).

IIéron Crabier.

Sgarza Ciuffetto.

Menghixedda groga C. M.

Sulla fronte, e sull alto della testa lunghe piume giallicie con delle striscie longitudinali nere; otto, o dieci piume stretle, e lunghissime partono dall'occipite, desse son bianche contornate d'un bordo nero; gola bianca; collo alto del dorso, e seapolari d'un rosso chiaro, il rimanente delle piume d'un bianco puro.

Di passaggio annuale in autunno. Abita come la specie precedente.

\section{SPEGIE CLXXII.}

Ardea Minuta (Linneo).

Heron Blongios.

Nonnotto.

Menghixedda pilica C. M.

Alto della testa, occipite, dorso, scapolari, penne secondarie delle ali, e coda d'un bel nero a riflessi verdastri; lati della testa, collo copertura delle ali, e tutte le parti inferiori d’un giallo rossiccio.

Di passaggrio qualche volta in aprile ma raro.

Il genere Sirone, Ardea trovasi dappertutto. 


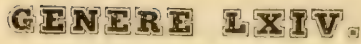 \\ Nitticona. Nxcticonax (Cuvier).}

Caratere generico. Becco un po" piu lungo della testa, o della medesima lunghezza, grosso, forte, largo, e dilatato alla base, mandibola superiore leggiermente piegata, ed inclinata verso la punta, che è spaccata ; mandibola inferiore dritla; narici laterali, longitudinali a poca distanza della base, nude, piazzate in una scanalatura, e coperte dalla membrana nuda ; piedi di mediocre lunghezza ; la nudita al di sopra del ginocchio molto piccola; larso piủ lungo del dito di mezzo, il dito esterno, e quello di mezzo riuniti da una membrana; unghie corte, quella del dito di mezzo dentellata. Questi uccelli hanno le stesse abitudini degli Aironi.

\section{SPECIE CLXXIII.}

Nycticorax Ardeola (Cuvier).

Bihoreau a Manteau.

Nitticora.

Menga niedda C. M.

Testa, nuca, dorso, e scapolari d'un nero a rillessi azzurrognoli, e verdastri, tre bianche, strettissime piume lunghe 6 o 7 pollici, sono piantate sopra la nuca; parti inferiori del dorso, ali, e coda d'un cenerino puro; fronte, gola, parte anteriore del rollo, e parti inferiori d'un bianco puro.

Di passaggio annuale in primavera, alcuni anni in gran numero, altri in scarso. Frequenta i luoghi paludosi.

Il genero Nillicora, Nyclirorax trovasi dappertullo. 


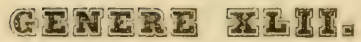

Fenicottero. Phomicopteres (Limeo).

Carattere generico. Becco grosso, forte, piu alto che largo, dentellato ai bordi, conico verso la punta, nudo alla base; mandibola superiore sensibilmente inclinata, piegata in punta sulla mandibola inferiore, che è più larga ; narici poste longitudinalmente in mezzo al becco forate da parte a parte presso quella specie di calotta, che forma lo spicolo superiore in parte coperte da una membrana; piedi lunghissimi; quattro dita, tre d'avanti uniti sino all'ugna da una membrana frastagliata, uno di dietro cortissimo, articolantesi altissimo sul tarso, unghie corte, piane; ali mediocri, primo, e secondo remigio piu lunghi di tutti. Nell'ordine di grandezza, questi uccelli seguirebbero immediatamente gli Struzzi, ed i Rea, poichè sono veramente le maggiori specie dopo quei giganti pennuti dell'Affrica, e dell'America. La vincono sovr'essi per l'immenso vantaggio di potersi alzare, e librare nelle alti regioni atmosferiche, facoltà niegata agli uccelli terrestri, che se ne compensano ì vero con una corsa talmente veloce, che non le si puó paragonare quella del piu agile quadrupede. Di raro questi uccelli incontransi isolati, ma redonsi ordinariamente in brigate assai numerose, e seguonsi in fila, e stringonsi con tale costanza, che mai sempre gli uni accanto agli altri strelli assieme alzansi, o discendono. Il loro nutrimento, consiste in ova di pesci, molluschi, piccole conchiglie, e semenze di vegetali marini. 


\section{SPECIE CLXXIV.}

Phoenicopterus Antiquorum (Temminck).

Flammant rose.

Fenicoltero o Fiamante.

Mangoni C. M.

Mangone, o zente rubia C.S.

Testa, collo, corla, e le parti interiori d'un bel roseo, ali d'un rosso vivo; dorso, e scapolari d'un rosso roseo ; remigj d'un nero profondo.

Di passaggio annuale, in alcuni anni ai primi d'agosto, in altri verso la meta, in altri funalmente ai primi di seltembre; partono alcune volte in marzo, altre in aprile, alcuni rimangono stazionarj, e vi nidificano. Ebbi varie volte dei novelli in peluria. Frequentano gli stagni. Nei r.00 e piu individui di questa specie che mi passarono fra le mani, trovai il ventricolo pieno di minutissime conchiglie del genere Cyclostoma, e Mitilus, ed una quantita di semi di piante marine di varie specie.

Il genere Fenicottero, Phoenicopterus trovasi dappertutto.

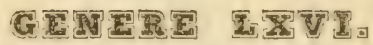 \\ Avocetta. Recurvirostra.}

Carattere generico. Becco lunghissimo, sottile, debole schiacciato in tutta la sua lunghezza, colla punta flessibile, currantesi in sủ, mandibola superiore soleata lateralmente, narici lineari, lunghe, situate alla base del becen: piedi gracili, lunghi ; tre dita d'avanti congiunte sino alla seronda articolazione con una 
membrana frastagliata, uno quasi di dietro articolantesi altissimo sul tarso; coscie mezzo nude: ali accuminate, il primo remigio è il più lungo. La conformazione del becco delle Avocette, il quale forma dalla punta alla base una specie di mezza luna, le cui due estremita stanno rivolte verso il cielo, basta per distinguerle dagli altri generi. Cosi poca consistenza ha questo becco verso la punta, che rassomiglia ad una fina linguetta membranosa ; e non di meno l'uccello lo confina assai profondamente nella melma per andarvi a cercare i vermi e le larve, delle quali come anche d'uova di pesci esso forma il suo alimento.

\section{SPECIE CLXXV.}

Recurvirostra Avocetta (Linneo).

Avocette à nuque noire.

Avocelta o Monachina.

Paisanu C. M.

Filippa C. S.

Sommita della testa, parte posteriore del collo, piccoli, e grandi scapolari, coperture alari, e remigj d'un nero profondo: tutte le altre parti d'un bianco perfetto.

Di passaggio annuale in autumno, partono alla fine di marzo, e tante volte verso la meta di aprile.

Il genere Avocetta, Recurvirostra, trovasi dappertulto.

\section{GENERE正雷。}

Begco spatula. Platalea (linneo).

Carattere generico. Becro lunghissimo, forte molto piallo, 
dilatato alla punta, rotondato in forma di spatola: mandibola superiore scanalata, transversalmente solcata alla base : narici poste alla superficie del becco, approssimate, oblunghe, coperte da una membrana : faccia, e testa in parte, o intieramente nuda: piedi lunghi, tre dita d'avanti riunite alla seconda articolazione da membrane profondamente frastagliate: il dito posteriore lungo, portante a terra : ali mediocri, larghe : il primo remigio poco più, o meno della lunghezza del secondo, che è il più lungo. Le Spatole vivono in societa, e si nutrono di piccole conchiglie lluviatili, dei piccoli rettili, ed inselli acqualici.

\section{SPECIE CLXXVI.}

Platalea Leucorhodia (Linneo).

Spatule blanc.

Spatola.

Gragalla C. M.

Tutle le piume d'un bianco puro, ecceltuate quelle del pelto, dove disegnasi una larga piastra di giallo rossicio, le di cui es tremita rimontano in una fascia sull' alto del dorso, ove si rimiscono: nudita degli occhi, e della gola d' un giallo pallido, ma debolmente tinto di rosso sulla base della gola suddetta.

Di passaggio annuale in autunno, e partono in maggio, frequentano i luoghi umidi in vicinamza delle saline, ed i nostri stagni.

Il genere Spatula, Platalea, trovasi dappertutto. 


\section{GENRI IXVII.}

\section{Im. Ims (Lacepede).}

Carattere generico. Becco allungato, gracile, arcuato, allungato alla base, depresso alla punta, che è rotonda, ed ottusa . mandibola superiore profondamente soleata in tulta la sua lunghezza : narici poste all'origine della parte superiore del becco, bislunghe, strette circondate da una membrana, che cuopre il solco: faccia, e di sovente una porzione della testa, e del collo nude: piedi assai gracili, snudati sopra del ginocchio : quattro dita, tre d'avanti, unite sino alla prima articolazione, uno di dietro lungo, e posante a terra : ali mediocri, il primo remigio più corto talvolta di molto del secondo, e del terzo, che sono i piu lunghi. Gli Ibi virono in societa, ma tosto accoppiali rimangono uniti, sinchè non renga qualche dispiacerole caso a rapire uno dei coniugi. Lavorano iusieme alla costruzione del nido, che la maggior parte della specie forma sopra alberi elevati, e di raro in mezzo a cespugli. Il loro nutrimento è di conchiglie, di pesciolini, di vermi, d'inselli, ed anche di erbe tenere.

\section{SPECIE CLXXVII.}

fbis Falcinellus (Temminck).

Ibis Falcinclle.

Ibi Falcinello, o Mignaluzzo.

Tadamu.

Testa d'un castagno nero, collo, petto, alto del dorso, e tulte lo parti inferiori d'un rosso castagno viro: dorso, groppone, 


\section{$-139$}

coperture delle ali, remigj, e penne della coda d'un verde nericcio a rillessi bronzati, e porporini.

Di passaggio annuale in autunno, e parte terminando la primavera. Si fa preda tanto di giovani, che di adulti, abita i nostri stagni, ed i luoghi paludosi, particolarmente alla parte di Elmas.

Il genere Ibi, Ibis, trovasi dappertutto, ove la temperatura non si abbassa sotto il zero

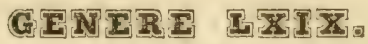

\section{Cumurlo. Numenius (Brisson).}

Carattere generico. Becco gracile, arcuato, compresso: la punta delle mandibole dura, leggiermente oflusa : la mandibola superiore sorpassante l'inferiore, scanalata circa a tre quarti della sua lunghezza: narici laterali, lineari, forate nella canalatura: faccia impiumata, spazio tra l'occhio ed il hecco coperto di piume: piedi gracili, nudi al dissopra del ginocchio: tre dita d' avanti, e uno di dietro gli anteriori riuniti quasi alla prima articolazione: il dito pollice articolato sul tarso, e toccante a terra : ali mediocri, il primo remigio piủ lungo di tulti. Questi uccelli vivono ne'luoghi aridi, e coperti di sabhia, ma sempre in ricinanza delle maremme.

Il loro nutrimento consisle principalmente in vermi di terra, insetti terrestri, ed acquatici, ed in lumache, e conchiglie. 


\section{SPECIE CLXXVIII.}

\section{Numenius arquatus (Latham).}

Courlis cendrè.

Chiurlo maggiore.

Curruliu imperiali C. M.

Tutte le piume d'un cenerino chiaro, delle macchic brune longitudinali sopra il collo, e sopra il pello con qualche penna tinta di rosso: ventre bianco con delle macchie longitudinali : piume del dorso, e scapolari nere nel centro, e bordate di rosso; coda di un cenerino chiaro rigato da fascie brune disposte transversalmente.

Comune fra noi, e vi nidifica. Abita gli stagni, el i lunghi sabbiosi.

\section{SPECIE CLXXIX.}

Numenius Phaeopus (Latham).

Courlis Corlieu.

Chiurlo piccolo.

Curruliu C. II.

Tulte le piume d'un cenerino chiaro, delle macchie brunce longitudinali sopra il collo, e petto: nel mezzo della testa una fiscia longitudinale d'un bianco gialliccio, accompagnata da riascun lato da un altra del doppio piu larga, e bruna : ventre, c basso-ventre bianchi, piume del dorso, e degli scapolari d'un bruno carico nel mezzo, e bordate d’un bruno piu chiaro: coda d'un bruno cenerino, rigata obliquamente con fascie brune.

Di passaggin in autunno, e parle in maggio, ma ì un por raro. 


\title{
SPECIE CLXXX.
}

\author{
Numenius Tenuirostris (Vieillot). \\ Courlis à bec gréle \\ Chiurlotello. \\ Curruliottu C. M.
}

Piume delle parti superiori a un dipresso come la specie precedente, ma più di bianco alla testa, alla nuca, ed ai bordi delle coperture delle ali: gola, parti anteriori del collo, petto, e tutto il rimanente delle parti inferiori bianco marcato longitudinalmente con striscie nere, che si fanno più grandi, e prendono la figura di cuore nella regione del ventre: coscie, basso-ventre, tulto il dorso d'un bianco risplendente; penne della coda rigate con larghe fascie bianche, ed altre nere piu strette.

Di passaggio come il precedente.

Il genere Chiurlo, Numenius, trovasi dappertutto.

\section{GENERI 通 \\ Beccacino. Tringa (Brisson).}

Carattere generico. Becco mediocre, o lungo, leggerissimamente arcuato, dritto o inflesso alla punta, flessibile in tutta la sua lunghezza, compresso alla base, ditatato, ed oltuso alla punta: le due mandibole quasi intieramente soleate: narici laterali coniche, forate nella membrana, che ricuopre il solco nasale in tutta la sua lunghezza: piedi gracili, nudi sopra del sinocchio; tre dita anteriori intieramente divise, talvolta quello 
di mezzo, el'esterno congiunto con una piccola membrana; un pollice articolato sul tarso: ali mediocri, primo remigio più lungo di tutti. Gli uccelli, che compongono questo genere sono essenzialmente viaggiatori; vanno quasi sempre riuniti in piccoli stormi, si veggono volteggiando dalla costa alla palude, limitare ad una cortissima apparizione, il loro soggiorno nei siti che visitano: pare anzi che la stagione degli amori, le cure che esige imperiosamente il bisogrno della riproduzione le arrestino contro voglia, nè supporre si puó che il solo timore di mancanza d'alimento sia la cagione determinante d'una vita tanto vagabonda; imperocchè siano per essi una sorgente quasi inesauribile le larve, i vermi, i moluschi, che loro offrono in abbondanza la melma, ed il limo. I heccacini vogliono una temperatura uniforme, e le stagioni determinano le emigrazioni loro dal nord al mezzodi, e dal mezzodi al nord, verso le due epoche equinoziali dell'anno: in tali emigrazioni le specie di riviera seguono regolarmente le spiaggie del mare, e quelle che soggiornano attualmente nelle paludi, dirigonsi secondo il corso dei fiumi e ruscelli

\title{
SPECIE CLXXXI.
}

\author{
Tringa Subarcuata (Temmink). \\ Bécasseau Cocorli, \\ Piovanello Panciarossa. \\ Beccacimu Arrubiastru C. M.
}

Faccia, sopracciglia, gola, copertura superiore della coda, ventre, e tutte le altre parti inferiori d'un bianco puro: una striscia bruna tra il becco, e l'occhio: alto della testa, dorso, scapolari, e coperture delle ali d'un bruno cencrino: piume della 
nuca strisciate longitudinalmente di bruno, e bordate di bianchiccio: parte anteriore del collo, e petto ugualmente bruno ma d'una tinta piu chiara; coda cenerina bordata di bianco.

Di passaggio annuale in autunno, e parte principiando l'estate, frequenta le sponde dei nostri mari, e stagni.

\section{SPECIE CLXXXII.}

Tringa Variabilis (Meyer).

Bécasseau Variable.

Piovanello Pancianera.

Beccacinu Brenti niedda C. M.

Gola, lo spazio tra il becco, e l'occhio, e tutte le parti inferiori d'un bianco puro: petto d'un cenerino bianchiccio: parti superiori d'un cenerino bruno: piume dell'alto del dorso, e scapolari d'un rosso vivo, hanno delle macchie sul loro mezzo.

Di passaggio come il precedente, frequenta però di preferenza gli stagni.

\section{SPECIE CLXXXVIII.}

Tringa Platyrhynca.

Bécasseau Platyrhinque.

Gambecchio Frullino.

Beccacinu differenti C. M.

Testa, ed occipite d'un bruno nericcio tagliato da due strette fascie longitudinali rosse; sopracciglia bianche a punti bruni : piume del dorso, e scapolari d'un nero profondo cordonate di 
rosso : gola, ventre e basso-rentre d'un bianco puro : parti anteriori, e laterali del collo d'un bianco rossiccio rariato da piccole striscie longitudinali brune : sui fianchi alcune grosse macchic brune.

Di passaggio qualche volta in primavera.

\section{SPECIE CLXXXIV.}

Tringa marillima (Brunnon).

Becassean Violet.

Piovanello Violelto.

Beccacinu Niedduz:u C. M.

Sommiti della testa, guancie, parti anteriori, e laterali del collo d'un ceucrino nericcio, piu oscuro sulla sommita della lesta, gola, contorno degli occhi, ed una piccola macchia tra l' occhio ed il becco d'un grigio bianchiccio : pelto grigio: piume del dorso, e scapolari d'un nero violelto a riflessi purpurei, bordate d' un rosso vivo: tutte le piume terminate di cenerino carico: coperture delle ali nericci e strisciate in cenerino chiaro : parti inferiori bianche: centro del ventre d'un bianco puro: groppone d' un nero profondo.

Di passaggio nell'inverno, abita sopra gli scogli, che bagna il mare alla parte di S. Elia, dove ne feci varie volte preda nella stagione suddelta, 


\section{SPECIE CLXXXV.}

Tringa minuta (Leisler).

Bècasseau Echasses.

Gambecchio.

Beccacineddu C. M.

Tutte le parli superiori cenerine con del bruno cenerino, lungo le bacchette, lati del petto d'un rosso cenerino; una striscia bruna tra l'occhio, ed il becco : centro del petto, gola, sopracciglia, parte anteriore del collo, e tutte le parti inferiori d'un bianco puro.

Comune fra noi, frequenta il bordo delle caselle delle Saline.

\section{SPECIE CLXXXVI.}

Tringa Cinerea (Linneo).

Bécasseau Maubèche.

Piovanello Maggiore.

Beccacinu e Zuruliu C. M.

Gola, centro del ventre, e basso-ventre d'un bianco puro, fronte, sopracciglia, parti laterali, ed anteriore del collo, petto, e fianchi egualmente bianchi, ma variali di piccoli tralti bruni longitudinali, e delle fascie transversali, ed in forma di zigzag d'un bruno cenerino: testa, collo, dorso e scapolari d'un cenerino chiaro.

Di passaggio in autunno, e parte in maggio, frequenta il bordo dei nostri mari.

Il genere Beccaccino, Tringa, trovasi dappertulto.

Nota. Il genere zi Combattenti, Machetes manea fra noi. 


\section{GENERE IXXII. \\ Totano. Totaxus (Bechstein).}

Carattere generico. Becco mediocre, o lungo, dritto, raramente curvo in alto, morbido alla base, duro, solido, e tagliente alla punta, compresso in tutta la sua lunghezza, terminato in punta aguzza; le due mandibole solcate solamente alla lor base; l'estremita della mandibola superiore leggerissimamente curvata sull'inferiore: narici laterali lineari, longitudinalmente fesse nel solco: piedi lunghi, gracili, nudi al di sopra del ginocchio; tre dita d'avanti, ed uno di dietro: il dito di mezzo riunito all'esterno quasi alla prima articolazione da una membrana, che qualche volta si prolunga quasi alla seconda articolazione. Sovente un rudimento di detta membrana nel dito interno, raramente una semi-palmatura: ali mediocri, il primo remigio più lungo di tutti. Questi uccelli vivono indistintamente sul bordo dei laghi, e delle riviere, come anche nei prati, che s'avvicinano alle acque dolci. Il nutrimento loro si compone d'inselti, vermi, e conchiglie, rarissimamente di pesci.

\section{Zlima Sirzinue.}

\section{Cavalieri (propriamente detli).}

Questi hanno le mandihole dritte, la punta della superiore curvata sull'inferiore: il dito di mezzo, e l'esterno uniti, o le tre dita più, o meno riunite. Il loro nutrimento consiste in vermi, insetti a elitri, ed in piccolissime conchiglie; questi abitano le acque dolci, ed i prati umidi. 


\section{SPECIE CLXXXVII.}

Totamus Fuscus. (Leisler).

Chevalier Arlequin.

Chio-Chio.

Zurruliu peis arrubius C. M.

Sommita della testa, nuca, dorso, scapolari, e coperture delle ali di un grigio cenerino: la gola, il petto, il ventre, il basso ventre, ed il groppone d'un bianco perfetto : fianchi d'un cenerino bianchiccio: una fascia nericcia tra il becco e l'occhio; guancie, parti laterali, ed anteriore del collo accordati tra il bianco, e cenerino.

Di passaggio in settembre, e parte in aprile e maggio.

\section{SPECIE CLXXXVIII.}

Totanus Calidris (Bechstein).

Chevalier Gambette.

Pettegola.

Zurruliu peis arrubius C. II.

La testa, la parte posteriore del collo, l'alto del dorso, gli scapolari, e le coperture alari di bruno cenerino: gola, lati della testa, parte anteriore del collo, e petto d'un bianco grigio; groppone, ventre, e basso-ventre d'un bianco puro.

Di passaggio in autunno, e parte in maggio. 


\title{
SPECIE CLXXXIX.
}

\author{
Totanus Stagnatilis (Bechstein). \\ Chevalier Stagnatile. \\ Piro Piro gambe lunghe. \\ Zurruliu peis longus C. M.
}

Sopracciglia, faccia, gola, centro del dorso, parte anteriore del collo, e del petto, e tutte le altre parti inferiori d'un bianco puro: nuca strisciata longitudinalmente di bruno, e bianco: altodella testa, e del dorso, scapolari, e grandi coperture delle ali d'un cenerino chiaro bordati di bianchiccio : lati del collo, e del petto bianchicci con delle piccole macchie brune.

Comunissimo fra noi, e vi nidifica, parte principiando l'estale.

\section{SPECIE CXC.}

Totanus Ochropus (Temminck).

Chevalier cul blanc.

Piro Piro cul bianco.

Zurruliu C. II.

Tutte le parti superiori d'un bruno leggiermente accordatu di olivastro, ed a riflessi verdastri : le piume del dorso degli scapolari, e delle coperture delle ali hanno dei piccolissimi punti bianchicci, che occupano i bordi delle barbe: una fascia bianca, ed altra bruna tra il beco e l'occhio: lutte le parti inferiori d'un bianco puro eccettuata l'anteriore del collo, e del petto, ore il bianco è variato da numerosissime striscie finissime, e ricinissime longitudinali brune: coda d'un bianco puro. 
Passa in primavera, ed in autunno, frequenta i luoghi pantanosi, lo cacciai varie volte ad Elmas.

\section{SPECIE CXCI.}

Totanus Glareola (Temminck).

Chevalier Silvain.

Piro Piro boscareggio.

Zurruliu peis birdis C. M.

Una stretta fascia tra il becco, e l'occhio: sommiti della testa, dorso ed ali d'un bruno carico: nuca, guancic, d'avanti del collo, petto, e fianchi d'un bianco sporeo variato irregolarmente di bruno disposto in onde, e striscia : sopracciglia, gola, centro del ventre d'un bianco puro.

Passa come il precedente, e vi nidifica.

\section{SPECIE CXCII.}

Totanus IIypolencos (Temminck).

Chevalier Guignelte.

Piro Piro piccolo.

Zurrulioteddu C. $\mathbf{I I}$.

Tulte le parti superiori d'un bruno olivastro a rillessi : tulte te piume defle ali, e del dorso rigate transversalmente con line fascie in figura di zigzag di un bruno nericeio : una piccola striscia bianca sopra degli cechi : gola, ventre, e le altre parli inferiori d'un bianco puro: lati del collo, e petto riggati longitudinalmente di bruno sopra un fondo bianco.

Passa l'inverno fra noi, e parte in marzo. 


\section{Secollon Sezinute.}

Cavalieri a becco rialzato.

Questi hanno le mandibole un poco curve in sú, dritte, $c$ quasi eguali alla punta: il becco grosso, e forte : il dito di mezzo, e l'esterno riuniti.

Il loro nutrimento si compone principalmente di pesci, e di piccole conchiglie bivalve. Vivono lungo i fiumi, ed i laghi d'acqua dolce.

\section{SPECIE CXCIII.}

Totanus Glottis (Bechstein).

Chevalier aboyeur.

Pantana.

Zurruliu, biccu grussu C. M.

Spazio della mandibola superiore all'occhio, gola, centro del petto, ventre, tutte le altre parti inferiori, ed il centro del dorso d'un bianco puro: testa, guancie, parte anteriore, e laterali del collo, e lati del petto rigate longitudinalmente di bruno cenerino, e di bianco: piume dell'alto del dorso, degli scapolari, e delle coperture delle ali d'un bruno nericcio contornate da una lunga bordura d'un bianco gialliccio.

Di passaggio in settembre e oltobre, parte in primavera.

Il genere Totano, Tolanus, trovasi dappertutto. 


\section{$-11+$ \\ GENERIXXIII \\ Bargia Linosa (Brisson).}

Carattere generico. Becco lunghissimo, molle, e flessibile in tulta la sua lunghezza, piu, o meno ricurvo in alto, depresso, pialto verso la punta: le due mandibole solcate lateralmente, terminate da una dilatazione, o sorta di cercine interno : narici laterali forate da parte a parte nel solco : piedi lunghi, gracili, con un grande spazio nudo sopra il ginocchio: Ire dita d'avanti, quello del mezzo riunito all'esterno da una membrana, che distendesi infino alla prima articolazione, un dito di dietro arlicolato sul tarso : ali mediocri, il primo remigio più lungo di tutti: le paludi, e le sponde limacciose formano l'unica abitazione delle Bargie. Il loro nutrimento è di larve, vermi, e piccoli molluschi.

\section{SPEGIE CYCIV.}

Limosa Melanura (Leisler).

Barge à queue noire.

Pittima reale, o Pantana.

Beccaccia de is cannas C. M.

Tulte le parti superiori d'un bruno cenerino uniforme, gola, parte anteriore del collo, petto, e fianchi d'un grigio chiaro: groppone nericcio: ventre, basso-ventre, parte superiore dei remigj, e base delle penne codali d'un bianco puro.

Di passaggio annuale in settembre, e parte principiando la primavera, frequenta i luoghi paludosi ove crescono le canne. 


\section{SPECIE CXCV.}

Limosa Rufa (Brisson).

Barge rousse.

Pittima piccola, o Pantana.

Beccaccia de is cannas C. M.

Sommita della testa, spazio tra l'occhio ed il becco, guancie, e tulte le parti del collo d'un cenerino chiaro marcate di numerosissime striscie longitudinali d'un bruno carico: le larghe sopracciglia, la gola, il petto, e tutte le parti inferiori d'un bianco il piu puro : parli superiori del dorso, scapolari d'un grigio cenerino, il rimanente del dorso, il groppone, e le coperture inferioridella coda bianche, ma variate da qualche macchia nericcia.

Di passaggio come la precedente, ma piu rara.

Il genere Bargia Limosa, si trova in tutte le regioni fredde, e temperate.

\section{GENER LXXIV.}

\section{Beccaccia. Scolopax (Illiger).}

Carattere generico. Becco lungo, dritto compresso, soltile, molle, colla punta rigonfia: mandibole solcate sino alla meti della loro lunghezza; punta della mandibola superiore più lunga dell'inferiore, formando la parte rigonfia un uncino: l'inferiore solcata nel mezzo, scanalata, e tronca all'estremita, narici laterali, situate alla base longitudinalmente, presso al bordo della mandibola, ricoperte da una membrana, piedi mediocri, gracili, appena un piecolo spazio nudo al di sopra del ginocehio: tre 
dita d'avanti, e uno di dietro; ali mediocri, il primo remigio quasi della medesima lunghezza del secondo, che è il più lungo.

Le Beccaccie abitano tanto nei boschi, in pianure, che in montagne, vi cercano gli asili piu selvatici, bene ombreggiati, dove il suolo costantemente umido, possa loro procurare in abbondanza i vermi limaci, e scarabei, dei quali si alimentano esclusivamente,

\section{दिके \\ Hrimu Sezinue.}

Beccaccie (propriamente delle).

Queste hamno le tibia impiumate quasi fino al ginocchio, ed abitano nei boschi tanto in pianura, che in montagna.

\section{SPECIE CXCVI.}

Scolopax Rusticola (Linneo).

Bécasse ordinaire.

Beccaccia ordinaria.

Beceaccia C. M.

Cabone Murdeiu C. S.

Parli superiori variate di rossiccio, di giallo, e di cenerino, e marcate da grandi macchic nere: parti inferiori d'un rosso gialliccio con dei zigzag bruni.

Arrivano in seltembre, e partono principiando l'estate.

Questa specie è comune in tulta l'Isola. 


\section{Sexoùn \$rzimie.}

Beccaccini.

Questi hanno la parte inferiore del tibia spogliato di piume. Vivono in pianura ne'luoghi paludosi, dove amano di nascondersi.

\section{SPECIE CXCVII.}

Scolopax maior (Linneo).

Bécassine double.

Beccaccino maggiore, o Croccolone.

Beccaccinu imperiali C. M.

Beccaccinu C. S.

La sommita della testa d'un nero diviso da una fascia di bianco gialliccia, sopracciglia di questo colore: parti superiori variate di nero, e di rosso chiaro: quest'ultimo colore disposto longitudinalmente: parti inferiori d'un rosso bianchiccio, ventre, e fianchi rigati da fascie nere.

Di passaggio annuale fra noi verso la mela di seltembre, frequenta i luoghi paludosi.

\section{SPECIE CXCVIII.}

Scolopax Callinago (Linneo).

Bécassine ordinaire

Beccaccino Reale.

Beccaccinu Riali C. II.

Beccaccinu C. S.

Parli superiori variate a un dipresso come la specie precedente: collo, e petto rigati longitudinalmente : fianchi rigati transver- 
salmente di bianco, e di nericcio: centro del ventre, e basso ventre di un bianco puro senzalcuna macchia.

Passa come il precedente, ma in più gran numero.

\section{SPECIE CXCIX.}

Scolopax Gallinula (Linneo).

Bécassine Sourde.

Beccaccino minore, o Frullino.

Beccaccinu C. II.

Ziriolu C. S.

La fascia che dalla fronte si prolunga insino alla nuca è di un nero macchiato di rosso; larghe sopracciglia gialliccie seguendo la direzione della fascia: parte anteriore del collo d'un cenerino bianchiccio marcata longitudinalmente d'un colore più opaco ; piume del dorso, e degli scapolari nere a riflessi verdi, e porporati, marcate da una fascia rossiccia disposta longitudinalmente.

Di passaggio in ottobre, e parte in maggio.

Il genere Beccaccia, Scolopax trovasi dappertutto.

Nota. La terza sezione dei Beceaccini Cavalieri manca fra noi.

\section{हुक्ष \\ GENERE $\mathbf{2} \mathbf{X} \mathbf{X}$. \\ Rallo. Ralus (linneo).}

Carattere generico. Becco più lungo della testa, gracile, leggerissimamente arcuato, o dritto, compresso alla base, cilindrico alla punta; la mandibola superiore solcata : narici laterali fesse longitudinalmente nel solco, formate a meti da una membrana, forate da parte a parte: piedi lunghi, forti, un piccolo spazio nudo al di sopra del ginocchio; tre dita d'avanti, c uno dietro, 
Ie dita anteriori divise, il posteriore articolato sul tarso : ali mediocri ritondate, il primo remigio un po' piu corto del secondo, terzo e quarto che sono i piủ lunghi. Questi amıano vivere nelle vicinanze, e nei bordi delle acque dolci, in particolare quelli che sono coperti di giunchi, erbe, ed arbusti. Il loro nutrimento consiste in vermi, insetti senza elitri, lumache, ed anche vegetali, c minute conchiglie fluviatili.

\section{SPECIE CC.}

Rallus Aquaticus (Linneo).

Rale d'eau vulgaire.

Gallinella.

Sturru de acqua C. M.

Puddighinu de aba, e de mala C. S.

Gola bianchiccia, lati della testa, collo, petto, e ventre d'un cenerino color di piombo: tutte le piume delle parti superiori d'un rosso bruno marcato nel centro d'un nero profondo rigato transversalmente da faseie bianche : coperture inferiori della cola bianche.

Comune, e stazionario, abita lungo i nostri fumi, e nidifica in mezzo alle giuncaglie.

Il genere Rallo, Rallus trovasi dappertutto.

\section{GENERE LXXVI.}

\section{Gallivole. Gallijula (Latham).}

Caraltere generico. Becco meno lungo della testa, compresso, conico, molto piu largen che alto alla sua base, mandibole di 
uguale lunghezza, compresse verso la punta, la superiore leggiermente curva : narici poste ai lati del becco verso il mezzo della sua lunghezza, fesse longitudinalmente, forate da parie a parte, ed in certa porzione coperte da una membrana: piedi lunghi: Ire dita d'avanti, ed uno di dietro, le anteriori lunghissime, c bordate da una membrana stretta : ali mediocri, concave : primo remigio piu corto del secondo e del terzo, questo, o il quarto più - lungo di tutli. Le Gallinole vivono più abitualmente sopra la terra che in seno agli stagni, ed alle paludi, dove nondimeno nuotano celermente, immergonsi con velocita: si soltraggono con destrezza agli sguardi dei cacciatori, ed alla persecuzione dei cani, correndo per mezzo ai giunchi, ed ai fusti paludosi. Si nutrono di vegetabili, vermi, ınsetti, molluschi, ed anche di pesciolini.

\section{खुछ \\ Yrimn Sezinne.}

La lisca della mandibola superiore dilatantesi tra le piume della fronte, e questa è placca frontale.

\section{SPECIE CGI.}

Gallinula Crex (Latham).

Poule d'eau de genet.

Re di quaglie, e Voltolino.

Su rei de is quallias $\mathrm{C}$. M.

Re de Trespotres C. S.

Un largo sopracciglio cenerino, si prolunga fin sopra i lati della testa: tutte le piume delle parti superiori d'un bruno ne- 
riccio, nel loro centro bordate lateralmente di cenerino, e terminate di rosso; gola, ventre, e basso-ventre bianchi : petto d'un cenerino olivastro; fianchi d'un rosso rigato in bianco.

Staziona fra noi, e se ne fa preda tanto nelle vicinanze dei fiumi, e ruscelli, che nei campi.

\section{SPECIE CCII.}

Gallinula Porzana (Latham).

Poulle d'ean marouette.

- Voltolino.

Puddixedda de acqua C. M.

Puddighinu de aba C. S.

Fronte, sopracciglia, e gola d'un grigio di piombo, lali della lesta d'un cenerino marcato di nero: le parti superiori d'un bruno olivastro, ma tutte le piume nere nel centro, e variate da piccole macchie, e da righe strette d'un bianco puro: petto, e parti inferiori d'un olivastro accordato di cenerino, e marcati con macchie bianche.

Passa fra noi principiando la primavera, frequenta le giuncaglie, e gli stagni.

\section{SPECIE CCIII.}

Gallinula Pusilla (Bechstein).

Poulle d'eau poussin.

Schiribollo.

Puddixedda de acqua C. M.

Puddighinu de aba C. S.

Gola, sopracciglia, lati del collo, petto, e ventre d'un grigio azzurrognolo, senza alcuna macchia, parte anteriore del collo, petto, e ventre d'un cenerino rossiccio; coscia, e basso ventre cenerino; parti superiori d'un bruno rossiccio; il grande spazio 


\title{
$-159-$
}

dell' alto del dorso variato da un piccolo numero di macchie bianche.

Passa, ed abita nelli stessi luoghi della precedente, ne feci preda dell'una, e dell'altra specie nel medesimo luogo.

\section{SPECIE CCIV.}

\author{
Gallinula Baillonii (Vieillot). \\ Poulle d'eau Baillon. \\ Pudixedda d'acqua. \\ Puddighinu de aba.
}

Gola, sopracciglia, lati del collo, pelto, e ventre d'un grigio azzurrognolo accordato sui lati del corpo coll'olivastro, sul quale veggonsi moltissime macchie bianche; parti superiori d'un rosso olivastro, variato sulla sommita della testa da striscie nere, sul dorso, e coperture delle ali una moltitudine di macchie bianche di forme variate contornate di nero profondo: fianchi, e basso ventre rigati transversalmente di lunghe fascie d'un nero profondo, e di strette fascie d'un bianco puro.

Di passaggio in primavera, ma di raro si fa redere fra noi, due soli individui è a me noto d'essere stati predati nella stagione suindicata.

\section{0 \\ Secolion Sizione.}

La lisca della mandibola superiore sporgente sulla fronte, e dilatantesi in una placca nuda. 


\section{SPECIE CGV.}

Gallinula Chloropus (Lalham).

Poulle d'eau ordinaire.

Sciabica.

Caboniscu de acqua C. M.

Pudda d'aba e Giaddina d'Eba C. S.

Testa, gola, collo, e tutte le parti inferiori d'un bruno olivastro carico : bordi esteriori delle ali, grandi macchie Iongitudinali sui fianchi, e coperture inferiori della coda di un bianco puro; base del becco, e la larga placca frontale d'un rosso vivo.

Stazionario fra noi, nidifica nelle giuncaglie che crescono in vicinanza dei nostri fiumi e ruscelli. Il genere Gallinella, Gallinula trovasi dappertutto.

\section{GENER IXXVIT}

Ponfirione o Theera, Porpuyrio (Brisson).

Carallere generico. Becco forte, duro, grosso, conico, quasi altreltanto alto che lungo, piu corto della testa: spigolo della mandibola superiore depresso, dilatantesi molto all'innanzi sul cranio; narici collocate ai lati del becco presso allo spigolo, sprofondate nella massa cornea, poco a presso rotonde, aperte da parte a parte; piedi lunghi c robusti; dita allungate; le anteriori affatto divise, fornite sui lati di piccole membrane strellissime; ali mediocri ; il primo remigio più corto del secondo, del terzo, e del quarto che sono regolarmente a scala. I Porlirioni, che diconsi anche Galline sultane, sono dei leggiadri uccelli acquatici, 
coperti per la maggior parte di colori turchini brillantissimi ; abitano i fiumi e le rive, ma piủ sovente i laghi, e le paludi, e i bassi fondi che nella stagione delle pioggie sono coperti d'acqua, la quale vi si ferma per una parte dell'anno. Nella lor maniera di vivere essi differiscono assai poco dalle Gallinelle, soltanto vedonsi per nutrirsi, andare in traccia di frulta, e di semi a preferenza delle foglie, e d'altre parti dei vegetali, non che del pesce cui si adattano il più d'ordinario le Gallinelle: quindi per tali motivi si trovano essi più spesso a terra, occupati in tale ricerca, e correndo attraverso i campi coltivati, e seminati di riso e di maiz soprattutto di quello che galleggianti, o immergentisi nel seno delle acque. Quantunque questi uccelli privi delle larghe membrane che fan le veci di remi ai palmipedi, pure sanno con destrezza ed agevolezza mirabile esercitare tutti i generi di nuoto, anzi ne spiegano piu ancora degli altri, e godono inoltre del vantaggio d'essere egualmente pronti ed agili al corso.

\title{
SPECIE CCVI.
}

\author{
Porphyrio Hyacentinus (Temminck). \\ Talève Porphyrion. \\ Gallina o Pollo sultano. \\ Puddoni C. M.
}

Un bell'azzurro turchino cuopre le guance, la gola, tutta la parte anteriore, e lati del collo: occipite, nuca, coscie e basso ventre d'un azzurro d'indaco molto carico, e poco vivo: petto, dorso, coperture delle ali, grandi penne delle medesime, e coda d'un azzurro d'indaco risplendente : placca frontale, e coronale, ed il becco d'un rosso vivo.

Di passaggio in aulunno; in alcuni anni numerosi, in altri 
scarsi, ed in altri rarissimi : la parte che piu sogliono frequentare è quella d'Elmas.

Il genere Porfirione, Porphyrio, trorasi nelle regioni calde e temperate dei due continenti.

\section{चजितिम \\ ORDINE XIV. \\ -0.50 당}

\section{PINNATIPEDI. PINNATIPEDES.}

Becco mediocre dritto, mandibola superiore leggiermente curva verso la punta: piedi mediocri : tarsi gracili o compressi, tre dita dinanzi unite da rudimenti di membrane, che bordano ciascuno dei lati : uno di dietro articolato intieramente sul tarso.

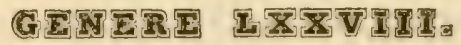 \\ Folaga .Fulica. (Brisson)}

Carattere generico. Becco mediocre, conico, grosso, dritto, compresso alla base, molto piì alto che largo: mandibola superiore leggiermente gonfia, ed inclinata verso l'estremita, dilatantesi sulla fronte in una placea nuda, l'inferiore angolosa, ambedue d'eguale lunghezza. narici laterali bislunghe, situate verso il mezzo del becco, forate da parte a parte, mezzo coperte da una membrana assai grossa : piedi assai allungati, grarili, 


\section{$-163-$}

sguerniti di piume sin sopra il ginocchio: quattro dita, le tre d'avanti lunghe riunite alla base, e guernite a ciascun lato da una membrana frastagliata in festoni : primo remigio più corto del secondo e del terzo che sono i piủ lunghi di tulti. Le Folaghe sono d'un naturale timoroso, il quale le fa stare nascoste tutto il giorno nei giunchi, e nelle canne, perche approffittando della facolta loro comune con molti altri uccelli di benissimo discernere gli oggetti all'oscuro, non percorrono le regioni dell'aria se non di notte, il che soltrae il loro volo ai nostri sguardi. Comunque siasi la facoltà di vedere nell'oscurila, che dinota sempre debolezza dell'organo della vista, è un beneficio per tulti gli uccelli che non si pascono fuorchè di pesci, e di rermi, poichè questi temendo lo splendore della luce, non si fanno solitamente vedere che in tempo di notte.

\section{SPECIE CGVII.}

Fulica Atra (Linneo).

Foulque Macroule.

Folaga.

Puliga C. M.

Puliga C. S.

Testa, e collo di un nero profondo: parti superiori d'un nero solor d'ardesia ; tutte le parti inferiori d'un cenerino azzurrognolo; la placca frontale larghissima d'un bianco puro.

Arrivano fra noi alle prime acque autunnali in grandissimo numero, partono in marzo, alcune ne rimangono stazionarie, e vi nidificano: ebbi varie volte i pulcini in peluria ai primi di giugno, ed esistono nel nostro Museo; si fa preda d'individui macchiati di bianco, e di bianchi affatto: un bellissimo individuo di quest'ultima lirrea senza la menoma macchia nera esiste pa- 
rimenti nel nostro Museo. Fra quelle che rimangono a nidificare fra noi se ne prendono alcune, che portano una piccola carnosila sopra la placca frontale. Abitano in tutti i nostri stagni.

Il genere Folaga, Fulica trorasi dappertutio.

Nolu. Il genere 79 Faloropo, Phaloropus manca fra noi.

\section{GEN⿴RI}

\section{Marangone. Podiceps (Latham).}

Caraltere generico. Becco di mediocre grandezza, ordinariamente più lungo della testa, dritto, conico, cilindrico ecompresso; mandibola superiore, drilla o curva improvvisamente verso la punta; narici situate verso il mezzo da ogni banda del becco, concave, bislunghe, aperte esternamente, e chiuse all'interno da una membrana comunicantesi dall'una all'altra; piedi riportati all' estremità del corpo; tarso compressissimo; quattro dita, tre d'avanti depressissime riunite alla loro base da una sola membrana che le circonda in festoni; l'esterno più lungo di tutti, pollice compresso, e festonato, articolantesi sulla faccia interna del tarso, e appoggiato in terza soltanto colla cima dell'unghia, che i al pari delle altre, larga, e depressa, ali corte, i tre primi remigj quasi uguali, ed i piu lunghi di tutti. Quantunque i Marangoni non abbiano le dita unite che per mezzo d'una semimembrana, non sono essi meno di tutti gli uccelli d'acqua i nuotatori piu snelli, e piit instancabili; la loro conformazione altronde indica assai che l'acqua fu loro assegnata come dimora abituale, ed anzi unica: imperocchè se si slanciano in aria, vi si sostengono con fatica, e sembra piultosto che sieno portati dai renti, di quello che seguano una direzione volontaria, che loro 


\title{
$-165-$
}

interdice forse la troppo brevita delle ali, relativamente al volume ed al peso del corpo. Sommergonsi con ammirabile destrezza, perseguitano e prendono in fondo dell'acqua i pesci che se ne stanno sicuri.

\section{SPECIE CCVIII.}

\author{
Podiceps cristatus (Latham). \\ Grébe II upé. \\ Suasso Comune. \\ Gangorra C. M. \\ Margone C. S.
}

Faccia bianca: sommita della testa, il ciuffelto schiacciato ed occipite, come la larga collana di ciascun lato delle guancie d'un nero lustro: questa tinta s' accorda col rossiccio sui lati della testa; tutte le parti inferiori d'un bianco lustro ed argentato: tutte le parti superiori brune e nericcie, un po' di rossiccio sui lati del petto, ed all'inserzione delle ali.

Tutto l' anno si fa vedere tra noi quest' uccello, particolarmente alla parte della Scaffa, ordinariamente isolato, o in coppie.

\section{SPECIE CGIX.}

Podiceps Auritus (Latham)

Grèbe Oreillard.

Suasso piccolo.

Cazzolu C. M.

Coaciou C. S.

La testa adorna d' un ciuffetto cortissimo, senza pennacchio nei gorgiera: la fronte d'un bruno rossiccio: tulte le parti supe- 


\section{$-166-$}

riori d'un bruno nericcio senza alcun lustro sul manto, di cui le piume sono sottilmente rigate d' un colore piủ chiaro: gola, e guancie d'un grigio di sorcio: dietro gli occhi una fascia longitudinale d'un rosso chiaro che s'unisce sulla parte anteriore del collo colorito di questa tinta: la testa d' un rosso chiaro marcato di striscie bianche: tutte le parti inferiori del corpo d'un bianco lustro marcato in fianchi di bruno chiaro, e di rosso.

Abbondante questa specie nel nostro golfo di Cagliari, tanto alla parte di Levante, che di Ponente, vi si vedono a piccole truppe, massime di inverno.

\section{SPECIE CCX.}

Podiceps Minor (Latham).

Grëbe Castagneux.

Tuffetto.

Accabussoni C. M.

Cabuzo, e Sorighe de riu C. S.

Gola, sommità della testa, e nuca d'un nero profondo, parte anteriore, e laterali del collo d'un castagno vivo; petto e fianchi nericci: il rimanente delle parti inferiori d'un cenerino nericcio: coscie e groppone tinto di rossiccio; parti superiori d'un cenerino lustro d'olivastro.

Vi staziona tutto l'anno, e si fa vedere nei nostri fiumi.

Il genere Marangone, Podiceps, trovasi dappertutto.

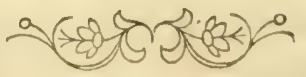




\section{$-167-$ \\ O R IOINE XV.}

\section{काषे \\ PALMIIPEDI-PALMIPEDES.}

Forma di becco assai varia, piedi corti, più o meno ritirati nell'addomine; tre o quattro dita d'innanzi munite d'una membrana intera, e piu o meno profondamente ritagliata ; uno di dietro (per quelli che ne hanno tre soltanto d'innanzi) articolato internamente sul tarso, e talvolta obliterato.

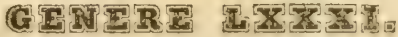

Rosdine Marmi, o Steria. Sterix (Limnco).

Carattere generico. Becco assai lungo, o piu della testa, quasi dritto, compresso, aftilato, tagliente, puntato, mandibole di eguale lunghezza, la superiore leggiermente incurvata verso li punta: narici poste verso la metà del becco, longitudinalmente fesse, forato di parte a parte; piedi piccoli, nudi al di sopra del ginocchio; tarso molto corlo; quattro dita le tre anteriori riunite da una membrana ritagliata, dito di dietro libero; unghie piccole, arcuate, coda piú o meno forcuta, ali molto lunghe, accuminate, il primo remigio piu lungo di tutti. 


\section{SPECIE CCXI.}

Sterna Caspia (Pallas).

Hirondelle de mer Tschegrava.

Rondine di mare maggiore.

Coa a biccu grussu C. M.

Fronte, sommità della testa d'un bianco puro: occipite variato di bianco e di nero; nuca, dorso, scapolari, e tutte le coperture delle ali d'un cenerino azzurrognolo: lati della testa, parte anteriore del collo, e tutte le altre parti inferiori di un bianco puro.

In un individuo, che ottenni nel decembre $18 \% 1$, quale esiste nel nostro Museo, trovai il ventricolo pieno di pesciolini chiamati in lingua Sarda, Conca de mallu, Lebius Caralitanus . . Ben di raro vedesi alla parte Meridionale dell'Isola, men di raro peró dalla parte Settentrionale, e specialmente sulle coste che guardano lo stretto di Bonifacio, ove la cacciò il mio amico Professor Cantraine, e dove il sig. Conte di Bellegarde Capitano delle Regie navi ne cacciò un individuo femmina co'suoi pulcini.

\section{SPEGIE CGXII.}

Sterna Cantiaca (Gmelen).

Hirondelle de mer caugek.

Becca pesci.

Caitta biccu nieddu C. M.

Rundine marina C. S.

Fronte, e sommità della testa d' un bianco puro, solamente 
variato verso l'occipite da tre piccole macchie nere, che occupano il centro delle piume: le lunghe piume dell' occipite d' un nero profondo, ma frangiato di bianco: una mezza luna nera d'avanti gli occhi: nuca, alto del dorso, tutte le parti inferiori, e la coda d'un bianco puro lustrissimo: dorso, scapolari, e coperture delle ali di un cenerino azzurrognolo molto chiaro.

Staziona fra noi, frequenta tanto il mare, che gli stagni.

\section{SPECIE CCXIII.}

Sterna Dougalli (Montaga).

IIirondelle de mer Dougalli.

Rondine di mare, zampe gialle.

Caitta peis grogus C. M.

Rundine marina C. S.

Sommità della testa, e tutta la nuca d'un nero profondo: dorso, scapolari, ed ali d'un cenerino chiaro: lati del collo, tutte le parti inferiori, e la coda d'un bianco puro: sul petto una leggiera tinta rossa.

Di raro comparisce fra noi, solamente d'estate.

\section{SPECIE CCXIV.}

Sterna IIirundo (Linneo).

Hirondelle de mer Pierre garin.

Rondine di mare.

Caixedda C. M.

Rundine marina C. S.

Fronte, sommità della testa, e le lunghe piume dell'occipite d'un nero profondo, parti posteriori del collo, dorso, ed ali d'un 
cenerino azzurrognolo: parti inferiori d'un bianco puro, eccettuato il petto, che ha una tinta cenerina.

Di passaggio annuale in primavera, e vi nidifica.

\section{SPECIE CCXV.}

Sterna Leucoptera (Temminck)

Hirondelle de mer Leucopter.

Mignattino, Zampe rosse

Caixedda peis arrubius $\mathrm{C}$. M.

Rundine marina C. S.

Testa, collo, alto del dorso, petto, ventre, coperture inferiori delle ali, e basso-ventre d'un nero profondo: parte inferiore del dorso, e scapolari d'un nero cenerino: piccole coperture medie delle ali, groppone, penne della coda, e le coperture tanto superiori, che inferiori d'un bianco perfetto.

Di passaggio in autunno, partono in primavera, alcune volte piì tardi, non è però troppo comune.

\section{SPECIE CCXVI.}

Sterna nigra (Linneo).

IIirondelle de mer épouvantail.

Mignattino.

Caixedda niedduzza C. M.

Rundine Marina C. S.

Testa, e parti superiori del collo d'un nero profondo: fronte, spazio fra il becco, e gli occhi, gola e tutta la parte anteriore del collo sino al petto d'un bianco puro: petto, ventre, e basso-ventre d'un nericcio cenerino: tulte le parti superiori, il groppone, e le penne della coda d'un cenerino azzurrognolo, e color di piombo.

Di passaggio in autunno, e vi nidifica, parte principiando 
l'estate. Abita il bordo del mare, ma di preferenza i nostri stagni, e le acque dolci.

\section{SPECIE CCXVII.}

Sterna minuta (Linneo).

Petite Ifirondelle de mer.

Fraticello.

Caixeddabianca C. M.

Rundine marina $\mathrm{C}$. S.

Fronte, ed una riga sopra degli occhi d'un bianco puro : una striscia longitudinale tra l'occhio, ed il becco: alto della testa, occipite, e nuca d'un nero profondo: dorso, ed ali d'un cenerino azzurrognolo puro: tutte le parti inferiori, groppone, e la coda bianchi.

Il loro arrivo è in primavera, e vi nidificano, partono in autunno.

Il genere Sterna, trovasi dappertutto: frequentano i bordi dei nostri mari, e gli stagni.

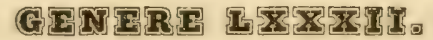 \\ Gabbiano, o Laro. Larus (Limneo).}

Carattere generico. Becco assai lungo, e forte, duro, compresso, e tagliente : mandibola superiore curva verso la punta, l'inferiore gonfia, formante un angolo tagliente: narici poste in mezzo del becco, ad ambi i lati fesse longitudinalmente, strette, e forate da parte a parte: piedi gracili, snudati fin sopra il ginocchio: tarso lungo: quattro dita, tre davanti interamente palmate; ed un pollice libero, corto, piu o meno visibile, articolante si altissimo sopra il tarso; rettrici d'uguale lunghezza : 
remigj lunghi, il secondo non oltrepassa che di pochissimo it primo. Sebbene questi uccelli siano d' una dolce apparenza, tuttaria pare che una vile ferocia formi la base del loro carattere, e le loro abitudini disgustanti ne fanno in diminutivo gli Avoltoj del mare. Muniti degli apparali di volo piu instancabile, spingono le loro escursioni molto innanzi nell'Oceano, e possono percorrere in pochissimo tempo estensioni di paesi assai considerabile; quindi essi trovansi su quasi tulte le coste, ore di sovente $\mathrm{i}$ loro stormi innumerabili ugualmente che le grida loro importanti ed ingrate stancano i marini ed i pescatori che li disprezzano quale inutile preda. La voracità dei Gabbiani è tanta. che veggonsi per abitudine contrastarsi un lembo di carognia infetta, rigettata dalle onde; l' accanimento che pongon a lacerarlo, del pari che a difenderlo cagiona tra essi le zuffe piu aspre, e non cessano se uno dei campioni non ismonti dalle sue pretensioni, il che non fa che allorquando esaurite ne sono le forze per le fatiche della lotta, o riportato abbia ferite talvolta mortali, perseguitato dal vincitore ne è ben presto lacerato e divorato. Corrono questi uccelli sopra la sabbia con assai celerita e leggerezza, volano con molta agevolezza e rapidita; nuotano poco, e piuttosto si abbandonano al bilanciamento delle onde per riposarsi dalle fatiche d'una lunga corsa aërea.

\section{SPECIE CGXVIII.}

Larus Glaucus (Brunn).

Mouelte Burgermeister.

Gabbiano Borgomastro

Cau Mannu, il giovane Gavina C. M.

Cao Marino C. S.

Testa, collo, tutte le parti inferiori d'un bianco puro: dorso, manto, cd ali d'un cenerino azzurrognolo chiaro. 
Comunissimo fra noi, nidifica nei nostri isolotti, se ne vede in grandissima quantita nell'isola di Serpentara.

\title{
SPECIE CCXIX.
}

\author{
Larus Argentatus (Brunn). \\ Moucte a manteau bleu. \\ Marino Pescatore. \\ Cau Marinu C. M. \\ Can Marino C. S.
}

Sommità della testa, regione degli occhi, occipite nuca, e lati del collo bianchi, ma tutte le piume marcate sul loro centro di una riga longitudinale d'un bruno chiaro: fronte, gola, tutte le altre parti inferiori, dorso, e coda d'un bianco perfetto: alto del dorso, e scapolari d'un cenerino azzurrognolo chiaro.

Capita di raro fra noi, e sempre d'inverno.

\section{SPECIE CGXX.}

\author{
Larus Marinus (Linneo). \\ Moutte à Manteau noir. \\ Mugnaiacrio. \\ Cau Marinu C. M. \\ Cao Marino C. S.
}

Sommitả della testa, regione degli occhi, occipite, e nucu bianchi, ma tutte le piume marcate nel loro centro da una striscia longitudinale d'un bruno chiaro: fronte, gola, collo, tutte le parti inferiori, dorso, e coda d'un bianco perfetto: alto del dorso, scapolari, e tutte le ali d'un nero opaco, che pare accordato coll'azzurrognolo. 


\section{$-174-$}

Accidentale fra noi è il suo passaggio; due soli individui ne sono stati predati d'inverno.

\section{SPEGIE CCXXI.}

Larus Flavipes (Linneo).

Mouette a pieds jaunes.

Zafferano mezzo moro.

Can C. M.

Cau Marinu C. S.

Sommita della testa, regione degli occhi, occipite, nuca, e lati del collo bianchi, ma tutte le piume macchiate nel centro da una striscia longitudinale d'un bruno chiaro: fronte, gola, tutte le altre parti inferiori, dorso, e coda d'un bianco perfetto: alto del dorso, scapolari, e le ali d'un nero carico, che pare accordato col cenerino.

Di passaggio fra noi in autunno, frequenta il bordo del mare, ed i nostri stagni.

\section{SPECIE CCXXII.}

Larus Audouini (Pyr).

Moucte Audouin.

Gabbiano Corso.

Cau C. M.

Cau Marinu C.S.

La testa, e la nuca bianche, coperte di moltissime striscie cenerine, petto, collo, ventre, fianchi, basso-rentre, groppone, e la coda d'un bianco perfetto: dorso, scapolari, coperture delle ali di un cenerino azzurrognolo. 
Durante l'inverno vedesi quest'uccello svolazzare nelle coste dell'isola della Maddalena da dove ne ebbi un bellissimo individuo.

\section{SPECIE CGXXIII.}

Larus Canus (Linneo)

Mouette a pieds bleu.

Gavina.

Cau e Gavina C. M.

Cao Marino C. S.

Testa, occipite, nuca, e lati del collo bianchi, ma seminati da numerosissime macchie d'un bruno nericcio: gola, tutte le parti inferiori, groppone, e coda d'un cenerino azzurrognolo puro.

Comparisce fra noi solamente negli inverni i più rigidi, e nelle cattive giornate si vede svolazzare nel nostro golfo di Cagliari.

\section{SPEGIE CGXXIV.}

Larus Tridactylus (Latham).

Mouette Tridactyle.

Gabbiano Terragnolo.

Cau C. M.

Cao Marino C. S.

Sommitá della testa, occipite, nuca, ed una parte dei lati del collo di un cenerino azzurrognolo uniforme; delle sottilissime striscie nere al davanti degli occhi : fronte, regione degli occhi, tutte le parti inferiori, groppone, coda d'un bianco perfetto; dorso, ali, e remigi d'un cenerino azzurrognolo puro.

Accidentalmente si fa vedere fra noi in primarera. 


\title{
SPECIE CGXV.
}

\author{
Larus Capisratus (Temminck). \\ Mouette à Masque brun. \\ Gabbiano Mezzano. \\ Cau C. M. \\ Cao Marino C. S.
}

Testa guernita d'un mezzo cappuccio di color bruno nerognolo, collo, petto, parte inferiori, e coda, d'un bianco perfetto: il petto, e ventre tinto di roseo: dorso, e manto di un cenerino azzurrognolo chiarissimo: le coperture inferiori delle ali d'una tinta di piombo.

In febbraio, e marzo si fa vedere fra noi questa specie, ma non comune.

\section{S PECIE CCXXVI.}

Larus Melanocephalus (Natteri).

Mouetle à capeuchon noir.

Gabbiano Corallino.

Cau, Conca de Moru C. M.

Cao Marino C. S.

Testa, collo, parti inferiori, e coda d'un bianco perfelto: dorso, ali, d'un cenerino azzurrognolo chiarissimo.

Giunge fra noi in primavera, lo vidi comune in questa slagione all' isola di San Pictro, e di Sant'Antisco.

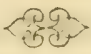




\section{SPECIE CCXXVII.}

Larus Atricilla (Linneo).

Mouette a capuchon plombs.

Gabbiano a Cappuccio color di piombo.

Cau conca de moru C. $\mathbf{M}$.

Cau marinu C.S.

Faccia, sommita della testa, gola, e lutte le altre parti inferiori, e la coda d'un bianco puro: avanti gli occhi un mezzo circolo d' un azzurro nericcio: questa linta mescolata di cenerino cuopre l'occipite, la parte superiore della nuca, e la regione dell'udito, fianchi leggermente linti di cenerino puro: dorso, e manto cenerino azzurrognolo molto cariro.

Passa di raro fra noi in primavera.

\section{SPEGIE CGXXVIII.}

Larus Ridibundus (Leister).

Moucte rieuse.

Gabbiano comune.

Can C. II.

Cane marinu C. S.

Testa, collo, e coda d'un bianco perfetto, ad eccezione d'una macehia nera avanti gli occhi, e d'altra grande nericeia sull'orificio delle orecchic: pelto, ventre, e basso ventre d'un bianco leggierissimamente tinto di rosa: dorso, scapolari, c tutte le coperture delle ali d'un cenerino nericcio.

Comunissimo fra noi in tulto l'anno e ri nidifica. 


\section{SPEGIE CCXXIX.}

Larus Tenuirostris (Bonaparte).

Mouette à bec grêle.

Gabbiano.

Can colori de rosu C. M.

Cau marimu C. S.

Testa, collo, e coda leggiermente tinto di roseo, le parti inferiori d'un bellissimo color di rosa; dorso scapolari, e tulte le coperture delle ali d'un nericcio azzurrognolo: remigii bianchi bordati, e terminati di nero : becco, e piedi di color vermiglio : iride dello steśso colore.

Questa specie che staziona fra noi venne descritta dal Principe di Musignano Carlo Bonaparte sotto il nome generico di Xema, che io non ho seguito perché attaccato al sistema del signor Temminck.

\section{SPECIE CCXXX.}

Larus Minutus Pallas .

Mouette Pigmée

Gabbianello

Caixeddu C. il.

Cau marinu C. S.

Fronte, spazio tra l'ocrhio, ed il becco, una gran macchía dietro gli occhi, gola, tutte le altre parti inferiori, e la coda d'un bianco perfetto: occipite, nuca, macchia aranti gli ofchi, e sull'orificio delle orecchie d'un nericcio cenerino; tutte le altre parti superiori d'un cenerino azzurrognolo chiaro. 
Pare che questa specie sia stazionaria fra noi, poichè il mio annico Professore Cantraine ne prese un individuo nel Golfo, di Cagliari nell' inverno del 1829. To ne ebbi in aprile ed agosto.

Il genere Gabbiano Larts, trovasi dappertulto.

Yola. Il genere 83. Stercorario, Lestrix, efl 84. Procellaria mancano fra noi.

\section{GWER LXXZV.

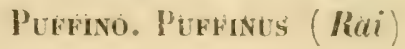

Caratter generico. Becen generahmente pii lungo della testa, gracile, e molto compresso alla punta: le due mandibole scanalate, repentinamente piegate verso l'estremita: l'inferiore piu, o meno ricurvata, e puntata. Yarici che si aprono in due tubi ravicinantisi alla superficie del becco; piedi mediocri, spesso lunghi, gracili: larsi compresi: tre dita all'innanzi: le unghie affatto palmate: un'unghia posteriormente puntutis sima che supplisce il pollice: ali assai distese; il primo remigio il piủ Jungo di tutti. I Puffini si nutrono di briceioli di celacci, di molluschi, di zoofiti, come pure di pesci. Rapiscono con destrezza, e senza mai immergersi nell' acqua la preda, che hanno posto da lungi presso alla superficie del mare, $c$ per cosi dire si servono del loro becco come d'un rampone. Volano con estrema rapiditi, portandosi con pari agevolezza contra vento, ed in poche ore pererrono spazi incredibili. Passano le notli aggruppati attormo a qualehe pumta di roccia. 


\section{SPECIE CCXXXI。}

Puffinus Cinereus (Linneo).

Puffin Cendré.

Berta Maggiore.

Giaurru C. M.

Notu. Giusta Linneo questa specie apparteneva al genere Procellaria, epperciò descritta sotto la denominazione di Procellaria Puffinus.

Testa, guancie, nuca, e dorso d'un cenerino chiaro: tutte le piume del dorso terminate da una zona piu chiara ancora: scapolari, ali, e coda di un cenerino nericcio, o color d'ardesia : ai lati del collo, e del petto delle onde di un cenerino chiarissimo: tutte le altre parti inferiori d'un cenerino chiarissimo : tutte le altre parti inferiori d'un bianco puro.

Comunissima fra noi, nidifica nelle fessure delle rocche, e scogli isolati, o prospicienti il mare.

\section{SPECIE CCXXXII.}

Puffinus Anglorum (Temminck).

Puffin Manks.

Berta minore.

Giaurru C. M.

Sommiti della testa, nuca, e generalmente tutte le parti superiori del corpo, le ali, la coda, le coscie d'un nero, che sembra lustro: tutte le parti inferiori d'un bianco puro: il nero, ed il bianco dei lati del collo si presentano in modo, che colle mezze tinte formano delle specie di lune crescenti.

Di rado si fa preda di questa specie, abita peró ne’lunghi, che frequenta la precedente. 
Vécello della tempesta. Tinsussunoma (Virg.)

Carattere generico. Becco più corto della testa, molto compresso alla punta: le mandibole scanalate repentinamente picgate verso l'estremila : l'inferiore compressa, scavata a grondaja formante un angolo: narici rimite in un sol tubo alla superficic del becco, ove lasciano velere due distinti orificii; piedi mediocri, spesso lunghi, gracili: tarso lunghissimo: tre dita d'avanti lunghe intieramente palmate, dito di dietro nullo rimpiazzato da un unghia molto puntuta ; ali lunghe: il primo remigio il pii lungo; coda quadrata, o leggiermente forcuta.

Le specie comprese sotto questo genere sono decisamente semi notturne. Di giorno fanno stazione nelle fessure delle rocche, o cacciano ai crepuscoli. Si nutrono di semenza di piante marine, e di piccoli molluschi, che cercano volando a piccola distanza della superficie delle acque.

Sieguono come il Puffino il corso delle navi.

\section{SPEGIE CCXXXIII.}

Thalassidroma Pelagica (Linneo).

Thalassidrome tempête.

Uccello della tempesia.

Pibinga C. II.

T'esti, dorso, ali, e coda d'un nero opaco; parte inferion d'uin nero di fuligine: una larga striscia transrersale d'un bianco puro sul groppone; scapolari, e penue secondarie delle ali terminate di bianco; coda, e remigi neri. 
Comunissima questa specie fra noi abita sopra goli scogli degli Isolotli, ove nidifica.

\section{G \\ Ocche. Anser (Brisson).}

Becco piì corto della testa, o di lunghezza uguale, forte, conico, elevalo alla base, coperto d'una cera, depresso verso la punta, rotonda, oftusa, unguiculata; bordi delle due mandibole dentellate in lame coniche, l'inferiore meno larga della superiore: narici laterali situate verso il mezzo del becco, forate d'aranti al becco di mediore lunghezas: piedi impiumati sino al ginocehio ritirati indietro, a tarso lungo : tre dita d'ananti inlieramente palmate, dito posteriore libero articolato sul tarso spogliato di membrana, o portante un rudimento: ali mediocri; il primo remigio uguale al secondo, o un po' piu corto.

Virono nelle praterie, e nelle paludi ; nuotano poco, e non si unthano vell'actuua ; nel volo le compagnie formano un angolo.

\section{SPECIE CCXXXIV.}
Anser Ferus (Latham).
Oie cendrée, o Premier.
Occa Pagliellana.
Oca de Mari C. M.
Dea Areste C. S.

Piume d'un cenerino chiaro; allo del dorso, stapolari, e grandi roperture delle ali d'un enerino bruno strisciato di bianchiccio; piccole coperture, lulli i hordi esteriori delle ali, o la base dei remigi di un cenerino bianchicrio; groppone cenerino; basso-ventre, e copertura inferiore della coda d'un hianco

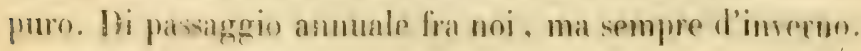




\title{
SPECIE CCXXXY.
}

\author{
Anser Segetum (Gemel). \\ Oie Vulgaire, ou Sauvage. \\ Occa Granainola. \\ Oca Silvatica C. M. \\ Oca Areste C. S.
}

Testa ed alto del collo d'un cenerino bruno; basso del collo. e le parti inferiori d'un cenerino chiaro ; alto del dorso, seapolari, e tutte le coperture delle ali d'un cenerino bruno strisciato di bianchiccio, groppone d'un bruno nericcio; basso ventre, e copertura inferiore della coda d'un bianco puro.

Di passaggio qualche volta in ottobre.

\section{GENERE IXXXII \\ Cigno Cygnus (Meyer).}

Becco di larghezza uguale in tutta la sua lunghezza, molto piu alto che largo alla base, depresso alla punta; le due mandibole dentellate a lamine transversali; narici oblunghe laterali forate verso il mezzo del becco; il collo sottile e molto lungo; piedi fuori d'equilibro, corti, tre dita d'aranti intieramente palmate, quello di dietro píccolo, e libero.

I Cigni hanno le acque in patrimonio, e con dei movimenti pieni di grazia, e di eleganza fanno pompa di quell'impero che a giusto titolo è stato loro accordato, essendo il piu bell'ormamento dei piani liquidi. Si nutrisenno di piante, ed inselti acquatici. 


\section{SPECIE CCXXXVI。}

Cygnus Musicus (Linneo).

Cygne Sauvage

Cigno Selvatico

Cignu o Cisini C. M.

Tutte le piume d'un bianco perfetto, ad eccezione della testa, e della nuca, che sono leggiermente tinte di gialliccio sporco; becco nero coperto alla base da una cera gialla, che circonda la regione degli occhi.

Di passaggio annuale in autunno, e parte in primavera. Di raro vedesi nelle acque di Cagliari, ma piu frequentemente in quelle d'Oristano.

\section{SPECIE CGXXXVII.}

Cygnus Olor (Linneo).

Cygne Tuberculẻ ou domestique.

Cigno Reale.

Cignu o Cisini C. M.

Tulte le piume d'un bianco puro, eccettuata, come nella precedente specie, la testa e la nuca ombreggiata appena d'un giallicio sporco; becco d'uguale larghezza in tulta la sua estensione, piu alto che largo; la mandibola superiore d'un bellissimo colore d'arancio, l'inferiore color di carne; entrambe sono dentellate, e bordate di nero, come pure le due lamine; la base coperta da una membrana nera, che s'estende anclie alle narici: piedi sfumati di nero. 
Alcune coppie se ne vedono fra noi d'inverno. Un bellissimo individuo ne fu predato ai 15 febbraio 1842 nello stagno di Cagliari detto Su Miamu, quale non mi fu dato di poter acquistare, ed avea la lunghezza totale di cinque piedi, e sette pollici.

\section{Anatre Anas (Limneo).}

Carattere generico. Consimili ai generi precedenti ad ecezione del becco, che questo l'ha troppo compresso e largo verso la punta, uguale, o pii lungo della testa, ed in alcune specie alquanto ricurvo in alto delle dentellature delle mantibole, che sono lunghe, e schiacciate, e del dito posteriore, che è libern affatto senza membrana.

Le specie, che cadono sotto questo genere ricercano sempre le imboccate dei fiumi, e non si mostrano mai sopra le accue di pieno mare. Vi passano solamente di volo. Queste anatre d'acqua dolce si tuffano raramente, e lo famno solo per sottrarsi alle disgrazie di cui possono essere minacciate. Emigrano lungo i fiumi, ed i laghi, e rimontano annualmente in massa le grandi riviere.

\section{SPECIE CCXXXVIII.}

Anas Tadorna (Linneo).

Canard Tadorne.

Anatra Volposa.

Anadiera. C. M.

Testa, e collo d'un verde opaco; parti inferiori del collo, cri- 
perlure delle ali, dorso, fianchi, groppone, e base della coda d'un bianco puro; scapolari, una larga fascia in mezzo del ventre, basso ventre, remigi, e l'estremità delle penne codali d'un nero profondo; un largo cinturone rosso cinge il petto, e rimonta sull'alto del dorso; spechio delle ali d'un verde porporato: coperture inferiori della coda, rosse.

Arrivano fra noi in decembre, e gennaio, partono dopo avervi nidificalo, alcune rimangono stazionarie.

\section{SPECIE CGXXXIX.}

Anas Boschas (Linneo).

Canard Sauvage.

Germanreale.

Anadi conca birdi C. M.

Anade conca irde, e Anade Reale C. S.

Testa, e collo d'un verde molto carico; un collare bianco al basso del collo; parti superiori rigate di zigzag finissimi d' un bruno cenerino, e d'un grigio bianchiccio; petto d'un color castagno carico; il rimanente delle parti inferiori d' un grigio bianco rigato di zigzag finissimi d'un bruno cenerino.

Stazionaria fra noi.

\section{SPECIE CCXL.}

Anas Strepera (Linneo).

Canard Chipeau.

Canapiglia.

Trigali C. M.

Tesia, cenllo marcato di punti bruni sopra un fondo grigio: 


\section{$-18 \%-$}

parti inferiori del collo, dorso, e petto marcati di mezze lune nere: scapolari, e fianchi rigati di zigzag, nericci e bianchi, similmente le coperture delle ali di un rosso castagno; grandi coperture, e groppone d'un nero profondo.

Di passaggio annuale in autumno, partono in maro e aprile.

\section{SPECIE CGXLI.}

Anas Acuta (Limeo).

Canard Pilet.

Codone,

Agu C. II.

Sommita delia testa variata di bruno, e nericcio, guancie, gola, ed alto del collo d'un bruno a gradazioni di violetto, e di porpora: sopra la nuca una fasciil nera bordata con due fascie bianche: parte anteriore del collo, el inferiore del corpo d'un bianco puro; dorso e tianchi rigati a zigzag neri, e cenerini; sughi seapolari delle lunghe macchie nere.

Di passaggio annuale in autunno, parte in aprile, alcumi amni piu lardi.

\section{SPECIE CCXLII.}

Anas Penelope (Linneo).

Canard Siffeur.

Fischione.

Busciu C. N.

Fromte d'un biano gialliccio; testa, collo d'un rosso castagno; 


\section{$-188-$}

faccia puntellata di nero; gola nera, petto di colore di feccia di vino; dorso, e fianchi rigati di zigzag neri, e bianchi : copertura delle ali, e parti inferiori bianche ; specehio delle ali composto di tre fascie, delle quali le laterali d'un nero profondo, e quella di mezzo verde, scapolari neri strisciati di bianco.

Di passaggio come la precedente.

\section{SPECIE CCXLIII.}

Anas Querquedula (Linneo).

Canard Sarcelle d'Été.

Mar:aiola.

Circuredda C. $\mathbf{I I}$.

Anadone C. S.

Sommità della testa nericcia ; una fascia bianca passa sopric gli occhi, e si dirige sopra la nuca; gola d' un nero profondo: testa e collo d'un bruno rossiccio seminate di piccoli punti bianchi; basso del collo, e petto scagliato a fascie nere; copertura delle ali d' un cenerino azzurrognolo ; specchio delle ali d' un verde cenerino bordato di due fascie bianche.

Di passaggio fra noi in decembre, e gennaio, c parte dopo avervi nidificato.

\section{SPECIE CCXLIV.}

Anas Crecca (Linneo).

Canard Sarcelle d'Iliver.

Alzavola.

Gircuredda C. M.

Anadone C. S.

Sommita della testa, guancie, collo d'un rosso rastigno; una 


\section{$-189$}

larga fascia verile s'estende dagli occhi sino alla nuca: parte inferiore del collo, dorso, scapolari e fianchi rigati alternativamente di zigzag bianchi, e neri; petto d'un bianco rossiccio variato di di macchie rotonde; ventre bianco, o d' un bianco gialliccio ; coperture delle ali brune; fascia bianca longitudinale sugli scapolari.

Di passaggio annuale nell'inverno, e parte dopo aver nidificato fra noi.

\section{SPECIE CCXLV.}

Anas Clypeata (Linneo).

Canard souchet.

Anatra Mestolone.

Biccangia C. II.

Testa e collo d'un verdastro carico a riflessi, petto di un bianco puro ; ventre, e fianchi d'un rosso castagno, dorso di un bruno nericcio; copertura delle ali d'un azzurro chiaro; scapolari d'un bianco marcato da punti, e macchie nericcie; specchio delle ali d'un verde carico.

Di passaggio annuale; arriva in autunno, e parte in aprile.

\section{SPEGIE CGXLVI.}

Anas Fusca (Linneo).

Canard double Macreuse,

Germano di mare.

Busciu de Ispagnia C. M.

Tulte le piume d' un nero profondo, e vellutato; sotto deglt occhi una mezza luna bianca; un piccolo specchio bianco sulle ali.

In qualche inverno si fa vedere fra noi, ma ben di raro. 


\title{
SPECIE CCXLVI.
}

\author{
Anas Rufina (Pallas). \\ Canard Sifleur Hupe. \\ Fischione Turco. \\ Piberoni C. M.
}

Testa con ciultio a piume setuse, guancie, gola, e parti supe-riori del collo d'un bruno rossiccio, o baio; parti inferiori del collo, petto, rentre, e basso-ientre d'un nero profondo; dorso, ali, e coda d'un bruno chiaro; fianchi, la giuntura delle ali, " base dei remigj, bianchi.

Di passaggio in marzo, ed aprile; piuftosto la specie è rara.

\section{SPECIE CCXLVII.}

Anas Marmorata (Temminck).

Canard Marbrẻ.

Garganella marmorata.

Trigali differenti C. M.

Testa ciuffeltata, sommità della medesima, circuito del becco. e tutto il collo bianchiccio macchiato con finissime striscie longitudinali: una gran macchia bruna-ovoide atiorno gli occhi: manto, dorso, scapolari, e penne codali di un bruno oscuro, con delle mezze lune di colore Isabella alle punte delle piume delle prime parti, egrandi macchie bianche con qualche gradazione cenerina nelle punte delle pinme delle seconde parti; pelto, fianchi, roscie, basso-sentre, or coperture della coda ondati 


\section{$-191$}

di bruno-chiaro sopra fondo bianchiccio appamnato: rentre piu chiaro, quasi impercettibile ondato di bruno chiarissimo.

Pare che questa specie faccia stazione fra noi, o in qualche parte vicina, poiche dessa finora non si i trovata in verun altra parte, ed è stata per la prima volta scoperta fra noi dal mio amico Professore Cantraine. Un altro individuo io ne ebbi in giugno del 1839, e mai piu finora potei ottenerne.

\section{SPECIE CCXLIX.}

Anas Marila

Canard Milouinan

Moretia grigia

Busciu C. M.

Tutta la testa, e la parte superiore del collo d'un nero it riflessi verdastri; parti inferiori del collo d'un nero profondo: alto del dorso, e scapolari d'un bianchiccio rigatı a grandi distanze con dei zigzag finissimi neri: ventre, e lianchi d'un bianco puro: basso-ventre rigato con dei zigzag bruni.

Accidentale è il passaggio di questa specie fra noi.

\section{SPECIE CCL.}

Anas Ferina (Limneo)

Canard Milonin

Moriglione

Cabarossu C. M.

Testa, c collo d'un rossiccio brillante: parti superiori del dorso, pello, e groppone d'un nero opano: dorso, scapolari coperture delle ali, fianchi, coscie, a basso ventre d'un co- 
nerino bianchiccio, rigato con numerosissimi, e vicinissimi zigzag d'un cenerino azzurrognolo: ventre bianchiccio variato di zigzag cenerini quasi impercettibili.

Di passaggio annuale in autunno; partono in aprile.

\section{SPECI E CCLI.}

Anas Leucophtalmos (Bechstein).

Canard a iris blanc ou myroca.

Moretla tabaccata

Nieduzzeddu C. M.

Testa, collo, petto, e fianchi d'un rossiccio vivissimo; all'intorno pel collo un piccolo collare d'un bruno carico: solto la mandibola inferiore una macchia angolare d'un bianco puro: dorso, ed ali d'un bruno nericcio a rillessi purpurei seminato di piccoli punti rossi: specchio alare bianco terminato in nero: ventre d'un bianco puro.

Piuttosto raro è il loro passaggio, ma sempre d'inverno.

\section{SPECIE CCLII.}

Anas Fuligula (Linneo).

Canard Morillon.

Morelta.

Niedduะzu C. M.

Testa con ciuffo a piume affilate, e lunghe, collo, e petto l'un nero a riffessi violetti, e verdi: dorso, ali, e groppone di un bruno nericcio a riflessi bronzati seminati di punti bruni: ventre, fianchi, e la fascia transversale sull' ala d'un bianco puro: basso ventre d'un bruno nericcio.

Comunissima fra noi è questa specie, che arriva in decembre, e parte dopo arervi nidificalo. 


\section{SPECIE-CCLIII.}

Anas Clangula.

Canard Garrot.

Qualtr occhi.

Anadi Furistera C. M.

Un grande spazio bianco alla radice del becco: il rimanente della testa, e la parte superiore del collo d'un verde porporato molto carico: parte inferiore del collo, petto, ventre, basso-ventre, fianchi, grandi coperture delle ali, ed una parte degli scapolari d'un bianco puro, dorso, groppone, ed una parte degli scapolari d'un nero profondo, coscie, e coda d'un nero cenerino. Arrivano fra noi in autumno, partono in primavera, ma in scarso numero.

\section{SPECIE CCLIV.}

Anas Leucocephala (Latham).

Canard Couronnè.

Gobbo ruginoso.

Titillonaxiu e Capocinu C. M.

Sommita della testa d'un nero profondo: fronte, guancie, gola, ed occipite d'un bianco puro: parti inferiori del collo, e nuca neri; petto, parti del corpo, e fianchi d'un bel rosso carico tagliato da fine linee in forma di zigzag d'un bruno nericcio: groppone d'un rosso porporato: coda nera: parti inferiori d' un bianco rossiccio lagliato transrersalmente con dei fini zigzag.

Questa specie non è molto numerosa, nè comune fra noi, pure vi nidifica. Ebbi varie volte in maggio i pulcini in peluria unitamenie alla madre. 


\section{CIISTREG}

\section{Mergo. Mergus (Linneo).}

Carattere Generico. Becco mediocre, o lungo, dritto, gracile, cilindrico, conico, piu, o meno allargato alla base: bordi delle due mandibole serratiforme, i denti acutissimi e diretti indietro, l'estremita della mandibola superiore adunchissima ed unguiculata; narici elittiche, trasforate da parte a parte, e longitudinalmente verso il mezzo dei due lati del becco; piedi corti, ritirati nell'addome; quattro dita, tre d'avanti intieramente palmate, l'esterno piu lungo delle altre, uno di dietro libero, articolato sul tarso, e che posa a terra sull'estremita; ali mediocri, il primo remigio uguale al secondo o soltanto un po' piủ corto.

Le specie comprese solto questo genere rassomigliano molto alle Anitre. Vivono sopra le açue ove nuotano, avendo per lo piu tutto il corpo sommerso ad eccezione della testa; si tuffano facilmente, e soventi, nuotano con estrema facilita, e si servono delle ali per ajutarsi nel nuoto. Volano con gran velocitá, e sostengono a lungo il volo. Si nutriscono principalmente di pesci e di anfibj.

\section{SPECIE CGLV.}

\section{Mergus Merganser (Linneo). \\ Grand Harle. \\ Smergo Maggiore. \\ Strallera C. M. \\ Cucumarzolu C. S.}

Testa, e parte superiore del collo d'un nero verdastro a riflessi; parte inferiore del collo, petto, ventre, basso-rentre, coperture delle ali, e gli scapolari i piủ lontani dal corpo d'un bianco puro con una gradazione di rosso gialliccio nelle parti inferiori: alto 
del dorso, e gli seapolari i più vicini del corpo d'un nero profondo ; piegatura delle ali nericcie.

Si vedono arrivare fra noi questi uccelli verso i primi d'ottobre, partono in aprile. Frequentano di preferenza gli stagni.

\section{SPECIE CCLVI.}

Mergus Serrator (Linneo).

Harle Huppé.

Smergo Minore.

Strallera C. M.

Cucumarzolu C.S.

Testa, ciuffo, e parte superiore del collo d'un nero verdastro a riflessi ; un collare bianco cinge il collo ; petto d'un bruno rossiccio marcato a macchie nere; all'inserzioni delle ali quattro. o cinque macchie bianche orlate di nero; specchio delle ali bianco, ma tagliato da due fascie transversali nere; alto del dorso, e scapolari d'un nero profondo, ventre bianco; coscie, e gropponi rigati di zigzag cenerine.

Passa, ed abita come la specie precedente, ma più comune.

\section{SPECIE CCLVII.}

Mergus Albellus (Linneo):

Harle piette.

Pesciajolo.

Scocalettu C. M.

Cucumarzolu C. S.

Una gran macchia d'un nero verdastro da ciascun lato del becco; una simile nera longitudinale sull'occipite ; ciuffo: sotto il collo gli scapolari, piccole coperture delle ali, e tutte le parti 
inferiori d'un bianco purissimo; alto del dorso, le due mezze lune, che si diriggono verso i lati del petto e dei bordi degli scapolari d'un nero profondo ; coda cenerina ; lianchi, e coscie variati di zigzing cenerini.

Passa in dicembre fra noi, i giovani sono comunissimi, e rarissimi gli adulti in perfetta livrea.

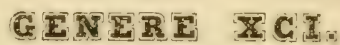

\section{Pellicano. Prelecanus (Linieo).}

Carattere generico. Becco lungo, dritto, largo, depressissimo, mandibola superiore appiattata, terminata in un'unghicta, o rampino durissimo, compresso, e molto currato: mandibola inferiore formata da due spigoli osseosi depressi, flessibili, riuniti alla punta; da questi due spigoli pende una pelle nuda in forma di sacco; faccia, e gola nuda; narici poste alla base del beero. fesse longitudinalmente; piedi forti, corti, tre dita d'avanti, quello di dietro s'articola internamente, ma sopra il medesimo piano degli altri, tutti sono riuniti da una sola membrana; unghie, quella del dito di mezzo senza dentellatura; ali di mediocre lunghezza; il primo remigio piu corto del seconto, che ì il pii lungo di tulti, le grandi copeeture alari, e le penne seconlarie. che s'approssimano di più al corpo sono dell'islessa lunghezza dei remigj.

I Pellicani sono uccelli grossissimi che virono indistintamente sui fummi, laghi, e lungo le coste mariltime. Il loro nutrimento ronsiste in pesci, dei quali si fanno una gran provrista nel rasto sacco che pende nella mandihola inferiore, da dove il nutrimento passa successivamente nell'esofago, a misura, che la digestione ì fatta. 


\title{
SPECIE CCLVIII.
}

\author{
Pelecanus Oriocrolalus (Linneo). \\ Pelican blane. \\ Pellicano. \\ Pellicamu.
}

Tulte le piume d'un bel bianco leggiermente tinto di russo chiaro, i remigj neri; faccia nudi d'un bianeo roseo; un mazzello di piume lunghe, ed aftilate all'occipite.

Di passaggio aceidentale; uno dei due individui presi all'inboccatura della Scalla in Cagliari esiste nel nostro Musev, don:s del fu Re Carlo Felice di gloriosa ricordanza.

Il genere Pellicano, Pelecanus trovasi dappertutto.

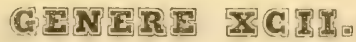

\section{Commoraxo. Carbo ( Meyer).}

Carattere generico. Becco assai lumgo, dritto compressu, rofondato di sopra; mandibola superiore soleata, curvatissima alla punta: l'inferiore compressa, più corta, oltusa, e poco curva : narici lineari, poste alla base del beceo, che e impegnato in una piccola membrana, la quale estendesi sulla gola, che è nuda, come anche la laccia; piedi corti, robusti, ritirati nel basso-rentre, yualtro dita riunite da una sola membrana, l'esterno più lungo di tulti, quello di dietro articolantesi internamente; l'unghia del rlito intermedio dentata a sega; ali mediocri, il primo remigio piiu corto del secondo, che i il pii lungo. I Cormorani sono wran 
consumatori di pesci, che perseguitano con una velocitả straordinaria. Tostochè questi uccelli hanno scoperto la preda che nuota pacificamente in seno al fiume, $o$ al mare, in un batter di ciglio s'immergono, afferrano con un remo la vittima, che indarno cercherebbe di sciogliersi dalla fatale membrana, e la riconducono ajutandosi coll'altro piede sulla superficie dell'onda; quivi con agile maneggio il pesce slanciato in aria, cadendo immediatamente colla testa in giu, viene ricevuto senza resistenza per parte delle pinne, i cui raggi sono allora naturalmente coricati indietro, nel gozzo dilatabilissimo dell'uccello.

\section{SPECIE CCLIX.}

Carbo Cormoranus (Meyer).

Grand Cormoran.

Marangone.

Crobu Anguiddargiu C. M.

Corvu Marinu C. S.

Sulla gola un largo collare bianco, e bianchiccio, le di cui estremita si estendono insino sotto gli occhi; sommita della testa, collo, petto, tutte le parti inferiori, e groppone d'un nero verdognolo a riflessi; sopra il collo dei piccoli tratt bianchicci, che sono quasi impercettibili; piume dell'alto del dorso, e delle ali d'un bruno cenerino, o color di bronzo nel mezzo orlate d'una larga fascia d'un nero verdognolo a rillessi.

Stazionario fra noi, abita tulti i nostri stagni, e si nutrisce a preferenza d'anguille, delle quali trova in abbondanza nei delti stagni. Veggonsi di notte le rupi di Sant'Elia coperte di Cormorami, tanto di questa specie, che delle seguenti.

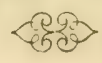




\section{SPECIE CCLX.}

Carbo Cristatus (Temminck).

Cormoran largup.

Marangone Largup.

Crobu Anguiddargiu C. M.

Corvu Marinu C. S.

Tutte le piume del piủ bel verde carico risplendente, e lustro: alto del dorso, scapolari, coperture, e penne delle ali d'un bel color di bronzo: ciascuna piuma è come incornicciata da una stretta fascia d'un bel nero, che sembra vellutato.

Stazionaria ì anche questa specie fra noi : abita come la precedente; ma si trattiene anche a pescáre sui bordi del golfo di Cagliari, questi non danno la preferenza alle anguille, come fa la sunnotala specie, li vidi varie volte pescare Labri, Spari, ed altri generi di pesci.

\section{SPECIE CCLXI.}

Carbo Leucogaster (Nobis).

Crobu brenti bianca C. M.

Becco affilato, sottile, l'apertura longitudinale del medesimo è di tre pollici, e nove linee, molto piu lungo della lesta : coda lunga, composta di dodici penne: tutte le parti superiori, cioe testa, collo, e groppone, sono d'un bruno leggiermente tinto di verdastro cangiante: le penne delle copritrici alari, e del dorso sono orlate d'una frangia biancastra, e lustra, remigj d'un nero opaco, coda dello stesso colore, ma bordata tanto nelle barbe esterne, che interne d'un bianco spore, tulte le parli inferiori 
d'un bianco puro, eccetto le parti esterne delle coscie, che sono sfumate di bruno nerognolo: la parte superiore del becco bruna , l'inferiore gialliccia: la piccola borsa gutturale dello stesso colore, piedi d'un giallo smorto: tarso, e parti inferiori delle dita nere, iride costantemente bianca. La lunghezza totale, cioè dalla punta del becco sino all'estremita della coda due piedi, e quattro pollici : lunghezza del tarso due pollici. I giovani di quesła specie sono d'un nericcio verdastro, nella parte superiore del corpo: l'inferiore bruna macchiata di bianco, l'iride bruna.

Staziona fra noi ; abita alla parte di Sant' Elia, non s'accompagna cogli altri, che vanno di giorno a pescare negli slagni, e fiumi, ma sta costantemente in quei dintorni : ne feci preda in varie stagioni, e le trovai sempre presso a poen nella suddescritta livrea.

Nidificano nelle fessure delle rupi del luogo suindicato : non potei linora ottenere le nova, a causa della pericolosa localita in cui le dispongono.

\section{SPECIE CCLXII.}

\section{Carbo Pygmaeus (Temminck).}

Carmoran Pygmée.

Cormorano Pigmeo.

Tulte le piume delle parti superiori del corpo d'un nero ceunino orlate d'una stretta fascia nera lustra, che pare vernicriala: testa, collo, e parti inferiori d'un nero veringnolo : sopria dengli occhi tre piccoli punti bianchi disposti in sopraceiglio.

Di passaggio accidentale. Due soli individui ne sono stati predati nel maggio 1831.

11 genere Cormorano. Curbo trovasi dappertullo.

Nola. Il genere 93 Pazzo, Sula manca fra noi. 


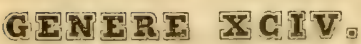

\section{Connibo. Colvurbus (Latham).}

Carattere generico. Becco mediocre, forte, dritto, molto puntuto, compresso, narici situate ad ambi i lati del becco, e presso la sua base, concare, bislunghe, semichiuse da una membrana, forate da parte a parte: piedi ritirati nel basso-ventre, tenendo il corpo fuor d' ecquilibrio: tarsi compressi: quattro dita, tre d'aranti lunghissime, intieramente pahnate: uno di dietro cortissimo, articolato sul tarso, portante una piccola membrana sciolta: unghie piatte: il primo remigio piu lungo di tutti: codi cortissima, e rotonda. I Colimbi perseguitano la lor preda sino negli abissi i piủ profondi, sono questi uccelli pesanti nel volo, e nel camminare, nuotano però con vivacitá maravigliosa, sommergonsi soprattutto con tanta faciliti, che si veggono spesso percorrere spazii lunghissimi, prima di ricomparire sulla superficie delle onde. Il loro nutrimento è di pesci, dei quali fanno una gran distruzione.

\section{SPECIE CCLXIII.}

\author{
Colimbus septentrionalis (Linneor). \\ Plongeon Cat marin. \\ Strologa piccola. \\ Gangorra differenti C. M.
}

Lati della lesta, yola, e lati del collo d'un cenerino vellutato, o color di sorcio: sommiti della testa mareata da macchice nere: orripite, parti posteriori, ed inferiori det collo marcate di righn" 
longitudinali nere, e bianche: sulla parte anteriore del collo una larga fascia d'un rosso castagno vivissimo : petto, e parti inferiori d'un bianco perfetto: fianchi, dorso, e tutte le altre parti superiori d'un bruno nericcio con delle piccole macchie bianche, e poco distinte.

Di passaggio accidentale fra noi. Un bellissimo individuo fu predato in uno dei nostri stagni nella primavera del 1838.

Il genere Colimbo, Colymbus, trovasi dappertutto.

Nota. Il genere 95 Guillemoto, Uria, manca fra noi.

\section{GENRE XCVI}

\section{Monmone. Monmon (Illiger).}

Carattere generico. Becco molto corto, piu alto che lungo, compressissimo, le due mandibole arcuate, scanalate transversalmente, smarginate verso la punta, lisea tagliente, alta piu del cranio : narici marginali lineari, quasi intieramente chiuse da una membrana nuda; piedi corti, ritirati nel basso-ventre, tre dita d'avanti del tutto palmate, senza pollice; unghie adunchissime : ali corte; il primo, e secondo remigio sono i piu lunghi; coda composta di dodici rettrici.

Il loro nutrimento consiste in pesciolini, molluschi, di crostacie, ed in mancanza di questi, di piante acquatiche 


\title{
SPECIE CCLXIV.
}

\author{
Mormon Fratercula (Temminck). \\ Macareux Moine. \\ Polcinella di mare.
}

Sommità della testa, tulte le parti superiori ed una larga collana che cinge il collo d'un nero profondo e lustro: guancie, una larga fascia sopra degli occhi, e gola d'un grigio chiarissimo, petto, ventre, e le altre parti inferiori d'un bianco puro.

Accidentale fra noi è il loro passaggio; un solo individuo ne fu predato vivo nell'inverno del $\mathbf{1 8 3 5}$, il quale si lascio avvicinare, e prendere colla mano senza la menoma difesa.

Il genere Mormone, Mormon trovasi nei mari del Nord.

\section{GENERE XCVI.}

\section{Pinguivo. Alca (Linneo).}

Carattere generico. Becco dritto. largo, compressissimo, assai curvo verso la punta; le due mandibole coperte alla metả da piume, solcate presso l'estremità ; la superiore adunca, l'inferiore che forma un angolo tagliente: narici collocate a ciascun lato del becco, alla metả lineari, quasi del tutto fermate da una membrana fornita di penne: piedi corti, ritirati nel basso-ventre; tre dita del tutto palmate; unghie poco adunche, ali corte, il primo remigio della lunghezza del secondo, o un po' piu lungo. 
Questi uccelli, celando i loro costumi, e le loro abitudini sui vasti mari vicini ai due poli sarebbero del tulto sconosciuti, se a lunghi intervalli le tempeste, e gli uragani non ne gettassern alcuni individui sui continenti, ed arregnacchè vi rimangono appena il tempo necessario per rimettersi delle fatiche della procella. Si nutrono come quelli del genere precedente.

\title{
SPECIE CCLXV.
}

\author{
Alca Torda (Linneo). \\ Pingouin Macroptere. \\ Gazza Marina.
}

Sommità della testa, nuca, lati del collo, e tulte le altre parti superiori d'un nero profondo; una fascia longitudinale d'un bianco interrolto da macchie brune va dal mezzo del beceo insino agli occhi : remigj d'un bruno nericcio; gola, parte anteriore del collo, petto, e tutte le parti inferiori d'un hianco puro; del bianco a macchie cenerine occupa i lati dell'occipite, ed una stretta fascia nera si disegna dietro gli occhi.

Di passaggio raramente fra noi, tre individui ne sono stati predati a mia notizia nello spazio di dieci anni.

II genere Pinguino, Alca trovasi presso i mari del Nord. 


\section{INDICE}

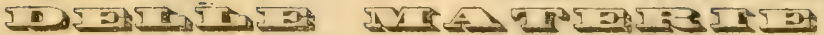

Conlenule nel presente Volume

DEDICA.

Prifazione .

\section{ORDINE I. - RAPACI.}

Gevere

))

I. Avoltoj

III. Gipaeli

IV. Falchi

V. Civette

)

)

ORDINE II. - OMINIVORI.

Gentere

VI. Corvi .

VII. Garrule . . . . . . . . . " 26

VIII. Spezza noci . . . . . . . . . . " ivi

IX. Pirrocoraccic . . . : . . . . . . ” 27

XI. Coraccie . . . . . . . . . " 28

XII. Rigogoli . . . . . . . . . . . 1) 29

XIII. Storni . . . . . . . . . . . . ” 30

XIV. Martinelli . . . . . . . . . . . 32

ORDINE III. - INSETTIVORI.

Gevere XV. Velic . . . . . . . . . . . ग 33

XVI. Pigliamosche . . . . . . . . . " 35

XVII. Merli . . . . . . . . . . . . ” 38

XVIII, Cincli. . . . . . . . . " 42

XIX. Becchi gentili . . . . . . . . . . ) 43

XX. Realtini . . . . . . . . " 53

XXI. Sericioli. . . . . . . . ग 54

XXII. Sassicole, . . . . . . . . . 》 55

XXIII. Accentore * . . . . . . . . . . ") 59

XXIV. Cutreltole . . . . ....... . 61

XXV. Spioncelle _. . . . . . . " 62 
ORDINE IV. - GRANIVORI.

GeNere

)

))

)

)

XXVI. Allodole

XXVII. Cingallegre

XXVIII. Zigole .

XXIX. Lossie .

XXXI. Fringuelli

ORDINE V. ZIGODATTILI.

GeNere XXXII. Cuculi. . . . . . . . . . . . \ 86

" XXXIII, Pichi. . . . . . . . . . . . " 87

» XXXIV. Torcicolli. . . . . . . . . . . 190

ORDINE VI. - ANISODATTILI.

GeNene XXXVII. Ticodroma . . . . . . . . . . \ 91

" XXXVIII. Bubole . . . . . . . . . . . ” 92

ORDINE VII. - ALCIONI.

Genere XXXIX. Merope . . . . . . . . . . . ” 94

)

XL. Alcedini . . . . . . . . . . 1195

ORDINE VIII. - CHELIDỎNI.

Genere XLI. Rondini. . . . . . . . . . " 97

)

XLII. Martinelli。 . . . . . . . . 》 100

))

XLIII. Succiacapre

ग) 101

ORDINE IX. - COLOMBI.

GeNere XLIV, Colombi . . . . . . . . . " 103

ORDINE X. - GALLINACCI.

Genere Xlix. Pernici . . . . . . . . " 106

ORDINE XI. - ALETTORIDI.

GENERE LI. Glareole . . . . . . . . . . . „ 109

ORDINE XII. =CORRIDORI.

Genene LII. Ottarde. . . . . . . . . . " 110

ORDINE XIII. - GRALLE.

Genere LIV. Edicnemi . . . . . . . . . . . 》 112

" LV. Sanderling . . . . . . . " 113 


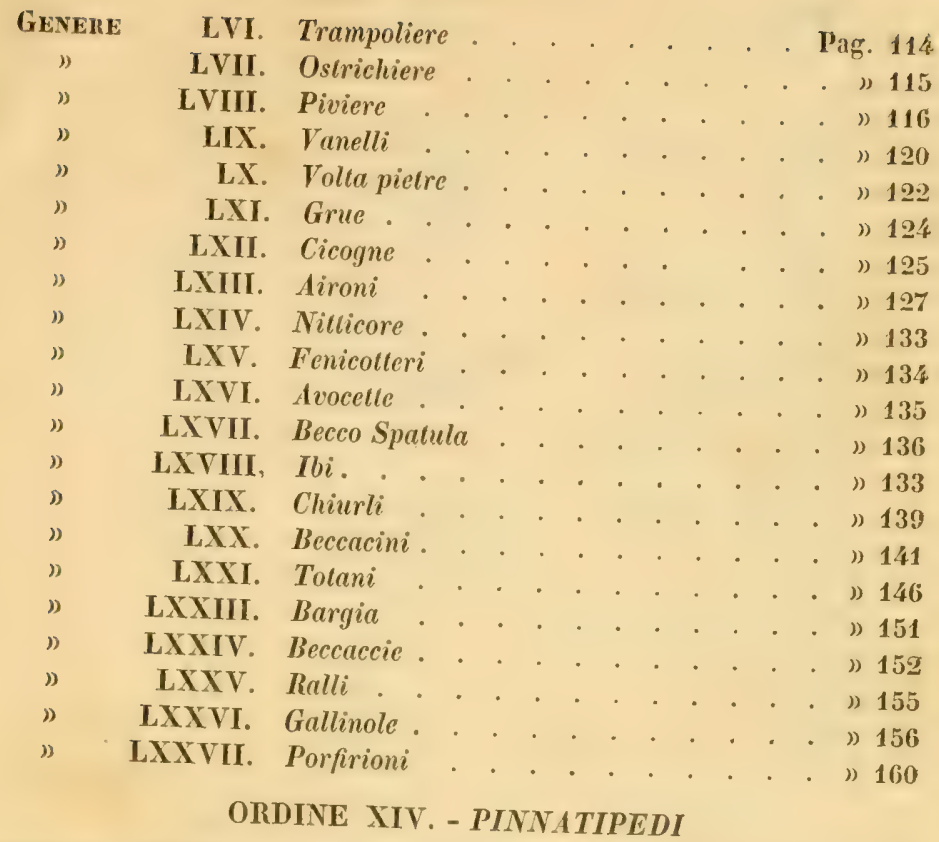

Genere LXXVIII. Folaghe

" LXXX. Marangoni

ORDINE XV. - PALAIPEDI.

Genere LXXXI. Rondini Marine.

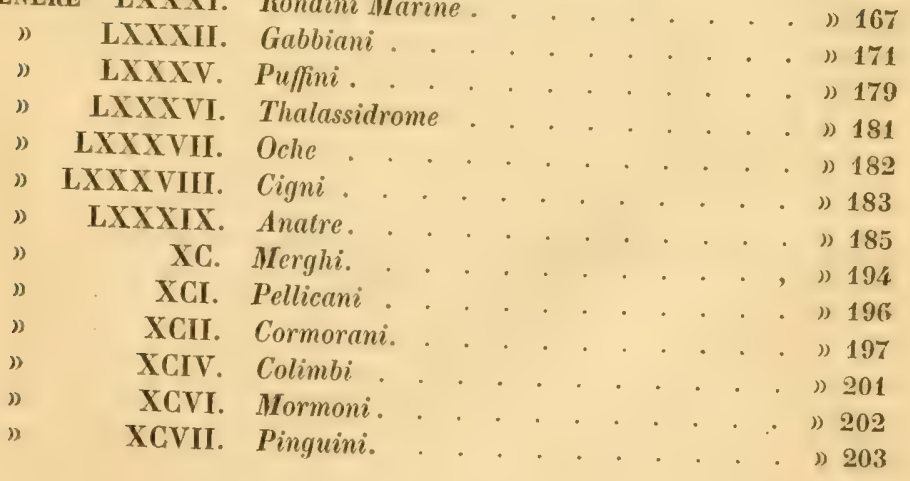



Holy. Lin.

\begin{tabular}{|c|c|c|c|c|}
\hline 2 & 20 & Dand & leggasi & Daud. \\
\hline 4 & I & Dand & ) & Daud. \\
\hline 7 & 13 & Astori & ) & Stori. \\
\hline 9 & 2 & Natheri & $n$ & Netteri. \\
\hline 13 & 2 & . . . & a!ggiungasi & Wolf. \\
\hline 18 & 10 & . . . & $"$ & Montagu. \\
\hline 21 & 10 & Brachiotos & legylyasi & Brachyotos. \\
\hline 39 & 14 & Musius & $\eta$ & Musicus. \\
\hline 41 & 16 & . . . & agyiungasi & Gmelin. \\
\hline 49 & 13 & . . . & $n$ & Gmelin. \\
\hline 54 & 8 & Bustein & leggasi & Brehem. \\
\hline 55 & 12 & Sericiolu & ") & Sericiolu. \\
\hline 83 & 2 & Coclebs & ) & Coelebes. \\
\hline 96 & 2 & . . . & agyiungasi & Linneo. \\
\hline 104 & 17 & . . . & $n$ & Linneo. \\
\hline 143 & 18 & . . . & $n$ & Temminck. \\
\hline 159 & 8 & . . . & $"$ & Schiribilla grigiata. \\
\hline$"$ & 9 & . . . & ) & C. M. \\
\hline$n$ & 10 & . . . & $n$ & C. 5. \\
\hline 168 & j & Con & leygasi & Cau. \\
\hline 169 & 9) & Montaga & ") & Montagu. \\
\hline 191 & $n$ & . . . & aygpiungasi & Linneo. \\
\hline 193 & 2 & . . . & ") & Linneo. \\
\hline 197 & 5 & . . . & ») & C. M. \\
\hline 205 & 19 & Martinetti & leggasi & Martino Roseo. \\
\hline
\end{tabular}

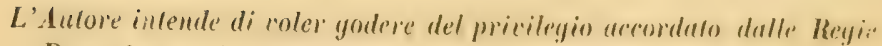
Patenti 28 febbraio 1826. 




$4 y+2+x-4$

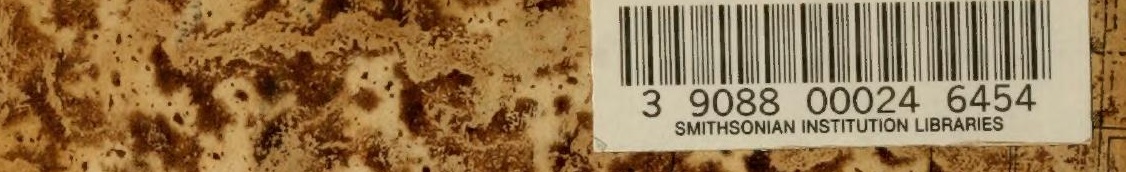
- 45 .

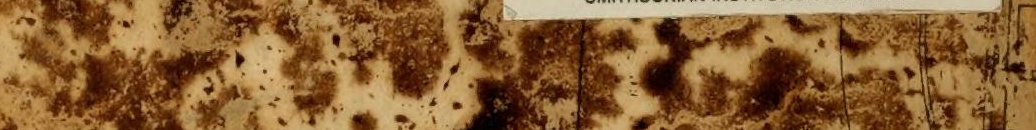

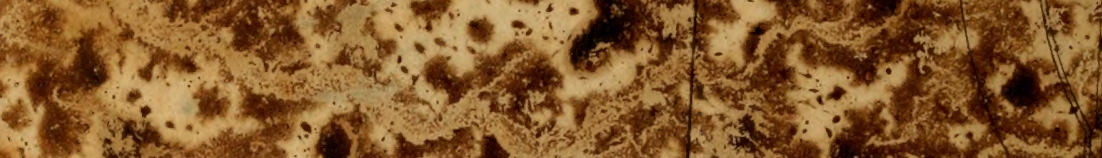
$40-1-27+231$

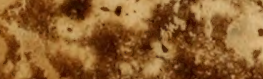
x.m. is:

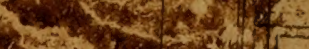
a. 3 in

$-1,2+5,4$ $x-10 \%$ x. $2+2$

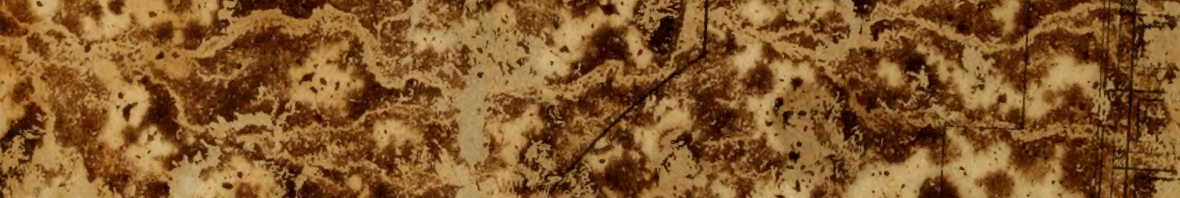

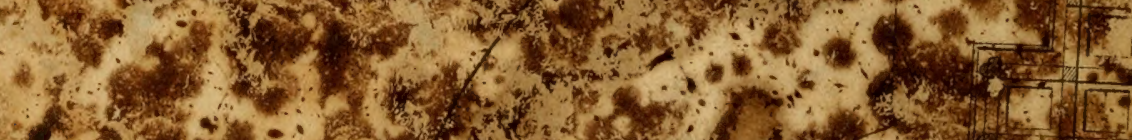
$\leq-4+1)$.

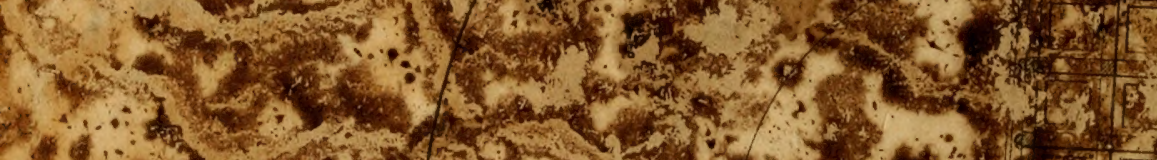

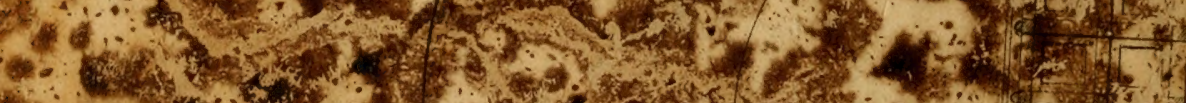
a.

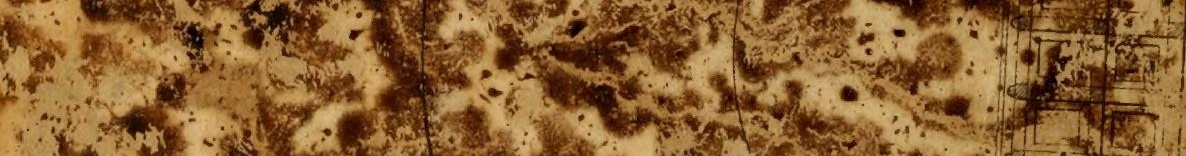

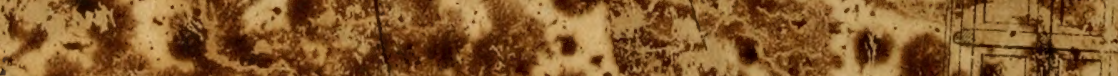
8.

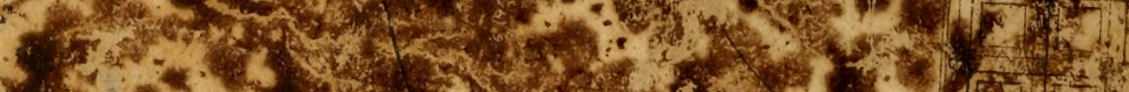

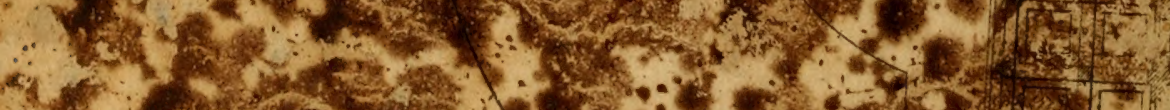

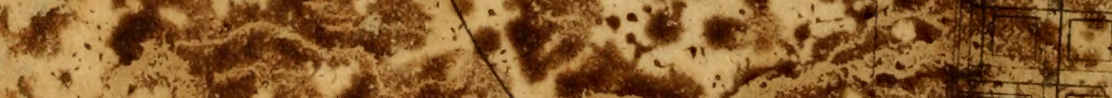

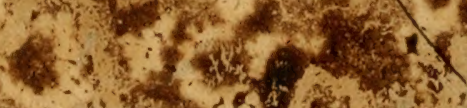

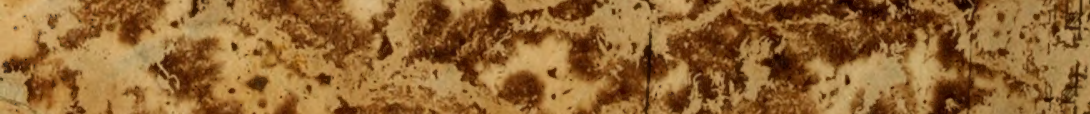

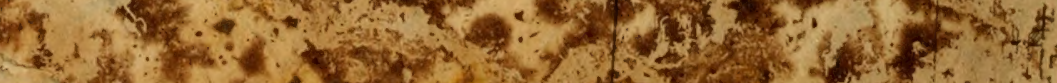

